\title{
On the Existence of Nash Equilibrium in Bayesian Games*
}

\author{
Oriol Carbonell-Nicolau ${ }^{\dagger} \quad$ Richard P. McLean ${ }^{\ddagger}$
}

August 2015

\begin{abstract}
We furnish conditions on the primitives of a Bayesian game that guarantee the existence of a Bayes-Nash equilibrium. By allowing for payoff discontinuities in actions, we cover various applications that cannot be handled by extant results.
\end{abstract}

Keywords: Bayesian game, discontinuous game, infinite game of incomplete information, behavioral strategy, distributional strategy, payoff security.

JEL classification: $\mathrm{C} 72$.

\section{Introduction}

Bayesian games are used extensively in many areas of applied research in Economics and other disciplines, and the notion of Bayes-Nash equilibrium is central in the analysis of such games. Furthermore, many economic models are most conveniently formulated as Bayesian games with infinitely many strategies and/or types (henceforth infinite Bayesian games) and discontinuous payoff functions. While some authors have studied infinite Bayesian games with continuous payoffs ( $c f$. Milgrom and Weber [50] and Balder [6], inter alia), there is very little work dealing with payoff discontinuities in Bayesian games (see Section 5.1 for a discussion of the papers of which we are aware). In this paper, we address the issue of existence of Bayes-Nash equilibrium in infinite Bayesian games with discontinuous payoffs and attempt to provide practitioners with a "toolkit" of relatively simple conditions that are useful in proving the existence of Bayes-Nash equilibrium in applied work.

To situate our results in the literature, we first recall some extant results in the case of complete information games. Building on previous work of Dasgupta and Maskin [23], Simon [71], and others, Reny [60] derived a number of existence results for games with discontinuous payoffs using various weakenings of upper semicontinuity of payoffs (such as Simon's [71] reciprocal upper semicontinuity or Dasgupta and Maskin's [24] upper semicontinuity of the sum of payoffs) and lower semicontinuity of payoffs (such as the notion of payoff security). If strategy sets are convex and payoffs are quasiconcave in own actions, then these weakenings

\footnotetext{
${ }^{*}$ We are indebted to Phil Reny for valuable insights. We thank seminar participants at the University of Chicago, SAET 2013, Université de Cergy-Pontoise, ITAM, and Ryerson University for their comments. The first author thanks the Becker-Friedman Institute of the University of Chicago for its kind hospitality and support.

${ }^{\dagger}$ Department of Economics, Rutgers University, 75 Hamilton Street, New Brunswick, NJ 08901, USA. Tel.: +848 228 2947. Fax: +732 932 7416. E-mail: carbonell@econ.rutgers.edu.

${ }^{\ddagger}$ Department of Economics, Rutgers University. E-mail: rpmclean@rci.rutgers.edu.
} 
of upper and lower semicontinuity can be applied to derive pure strategy existence results. ${ }^{1}$ The mixed extension of a game will satisfy the convexity and quasiconcavity assumptions so these pure strategy existence results can be applied to the mixed extension if the mixed extension itself satisfies the Reny weakenings of upper and lower semicontinuity. It is however useful to identify conditions on the primitives of a complete information game implying that the mixed extension will satisfy the Reny conditions (therefore implying the existence of a mixed strategy equilibrium). Such conditions are typically easier to verify and one such condition, called uniform payoff security in Monteiro and Page [51], guarantees that the mixed extension of a strategic game is payoff secure.

In studying games with incomplete information, one can formulate the existence question in terms of behavioral strategies (i.e., measurable functions that map a player's type to a probability measure on actions as in, e.g., Balder [6]) or in terms of distributional strategies (i.e., probability measures on the Cartesian product of a player's type and action spaces, as in Milgrom and Weber [50]). These formulations are interchangeable in the sense that an equilibrium in behavioral strategies exists if and only if an equilibrium in distributional strategies exists. While we work with behavioral strategies, we briefly outline (in Subsection 5.2) the complementary approach in terms of distributional strategies. In both the behavioral strategy formulation and the distributional strategy formulation of the strategic form game constructed from the primitives that define a game of incomplete information, the strategy sets will be convex and the payoffs will be affine in strategies. Thus, if the spaces of behavioral or distributional strategies are endowed with topologies in which they are compact subsets (of a topological vector space), then Reny's existence result applies immediately if the respective strategic form games satisfy the Reny relaxations of upper and lower semicontinuity. However, as in the complete information case, it is useful to identify conditions on the primitives of an incomplete information game ensuring that the behavioral or distributional forms will satisfy the Reny criteria. This is what we accomplish in this paper. First, we identify a condition on primitives (also called uniform payoff security below) that implies payoff security in the corresponding behavioral strategic game forms, thereby extending the result in Monteiro and Page [51] to the the incomplete information framework. Second, we show that upper semicontinuity in actions of the sum of payoffs at every type profile is sufficient to guarantee the upper semicontinuity of the sum of payoffs defined on behavioral strategies. These two observations, together with Reny's results, give our first main existence result (Theorem 1 ).

An alternative approach to equilibrium existence in complete information games employs the Nikaido-Isoda aggregation function and relies on the notions of diagonal transfer continuity and diagonal transfer quasiconcavity. This approach was introduced in Baye et al. [13]. Recently, Prokopovych and Yannelis [58] have proposed the notion of uniform diagonal security, which, in the aggregation function approach, plays the role of uniform payoff security. In particular, uniform diagonal security is an assumption on primitives that guarantees the existence of mixed strategy equilibria. Here, we present an extension of uniform diagonal security, defined on the primitives of a game with incomplete information, that implies diagonal transfer continuity in the corresponding behavioral or distributional strategic game forms. This allows us to prove our second main existence result (Theorem 2).

Our existence results provide easily verified conditions, covering applications that cannot be handled by the extant literature. This is illustrated in the context of common value auctions, Cournot competition, Bertrand-Edgeworth competition, and imperfectly discriminating contests.

\footnotetext{
${ }^{1}$ For an excellent survey of this literature, see Carmona [19].
} 
The paper is organized as follows. Section 2 introduces notation and definitions needed in the development of the main existence results, which are presented in Sections 3 and 4 and illustrated in the context of various applications in Section 6. Subsection 5.1 discusses related literature and Subsection 5.2 outlines an essentially equivalent approach to the existence problem using a formulation in terms of distributional strategies.

\section{Preliminaries}

Throughout the paper, the following definitions will be adopted. If $S$ is a compact metric space, then $\mathscr{B}(S)$ will denote the $\sigma$-algebra of Borel subsets of $S$, and $\Delta(S)$ will represent the set of Borel probability measures on $S$. In addition, $C(S)$ will denote the set of all real-valued continuous maps on $S$.

\subsection{Games}

Definition 1. A strategic-form game (or simply a game) is a collection $G=\left(Z_{i}, g_{i}\right)_{i=1}^{N}$, where $N$ is a finite number of players, $Z_{i}$ is a nonempty set of actions for player $i$, and $g_{i}$ : $Z \rightarrow \mathbb{R}$ represents player $i$ 's payoff function, defined on the set of action profiles $Z:=\times_{i=1}^{N} Z_{i}$. The game $G$ is called a topological game if each $Z_{i}$ is a topological space.

Throughout the sequel, given $N$ sets $Z_{1}, \ldots, Z_{N}$, we adhere to the following conventions, which are standard in the literature, even though they sometimes entail abuses of notation: for $i \in\{1, \ldots, N\}, Z_{-i}:=\times_{j \neq i} Z_{j}$; given $i$, the set $\times_{j=1}^{N} Z_{j}$ is sometimes represented as $Z_{i} \times Z_{-i}$, and we sometimes write $z=\left(z_{i}, z_{-i}\right) \in Z_{i} \times Z_{-i}$ for a member $z$ of $\times_{j=1}^{N} Z_{j}$.

Definition 2. A Bayesian game is a collection

$$
\Gamma=\left(\left(T_{i}, \mathscr{T}_{i}\right), X_{i}, u_{i}, p\right)_{i=1}^{N},
$$

where

- $\{1, \ldots, N\}$ is a finite set of players;

- $\left(T_{i}, \mathscr{T}_{i}\right)$ is a measurable space, where $T_{i}$ is player $i$ 's nonempty type space ${ }^{2}$

- $X_{i}$ is player $i$ 's action space, a nonempty compact metric space;

- $u_{i}: T \times X \rightarrow \mathbb{R}$, where $T:=\times{ }_{i=1}^{N} T_{i}$ and $X:=\times{ }_{i=1}^{N} X_{i}$, represents player $i$ 's payoff function, assumed bounded and $\left(\left[\otimes_{i=1}^{N} \mathscr{T}_{i}\right] \otimes\left[\otimes_{i=1}^{N} \mathscr{B}\left(X_{i}\right)\right], \mathscr{B}(\mathbb{R})\right)$-measurable; and

- $p$ is a probability measure on $\left(T, \otimes_{i=1}^{N} \mathscr{T}_{i}\right)$ denoting the common prior over type profiles.

For each $i \in\{1, \ldots, N\}$, let $p_{i}$ be the marginal probability measure induced by $p$ on $T_{i}$, i.e., the probability measure on $\left(T_{i}, \mathscr{T}_{i}\right)$ defined by

$$
p_{i}(S):=p\left(S \times T_{-i}\right), \quad \text { for every } S \in \mathscr{T}_{i} .
$$

For each $\left(T_{i}, \mathscr{T}_{i}\right)$ and $X_{i}, \mathscr{C}\left(T_{i}, X_{i}\right)$ will denote the space of integrably bounded Carathéodory integrands on $T_{i} \times X_{i}$, i.e., the functions $f: T_{i} \times X_{i} \rightarrow \mathbb{R}$ that are integrably bounded and $\left(\mathscr{T}_{i} \otimes \mathscr{B}\left(X_{i}\right), \mathscr{B}(\mathbb{R})\right)$-measurable with $f\left(t_{i}, \cdot\right) \in C\left(X_{i}\right)$ for each $t_{i} \in T_{i} \cdot{ }^{3}$

If $\left(\left(T_{i}, \mathscr{T}_{i}\right), X_{i}, u_{i}, p\right)_{i=1}^{N}$ is a Bayesian game, we will write $\mathscr{T}$ for the product $\sigma$-algebra $\otimes_{i=1}^{N} \mathscr{T}_{i}$, and $\Delta(T, \mathscr{T})$ will denote the set of probability measures on the measurable space $(T, \mathscr{T})$.

\footnotetext{
${ }^{2}$ Observe that we do not impose any topological structure on $T_{i}$.

${ }^{3}$ An $\left(\mathscr{T}_{i} \otimes \mathscr{B}\left(X_{i}\right), \mathscr{B}(\mathbb{R})\right)$-measurable function $f: T_{i} \times X_{i} \rightarrow \mathbb{R}$ is integrably bounded if there exists a $p_{i}-$ integrable function $\varphi$ satisfying $\left|f\left(t_{i}, x_{i}\right)\right| \leq \varphi\left(t_{i}\right)$ for all $\left(t_{i}, x_{i}\right) \in T_{i} \times X_{i}$.
} 
Definition 3. Let $\Gamma=\left(\left(T_{i}, \mathscr{T}_{i}\right), X_{i}, u_{i}, p\right)_{i=1}^{N}$ be a Bayesian game. A pure strategy for a player $i$ in $\Gamma$ is a $\left(\mathscr{T}_{i}, \mathscr{B}\left(X_{i}\right)\right)$-measurable map $s_{i}: T_{i} \rightarrow X_{i}$ with the interpretation that, upon learning her type $t_{i} \in T_{i}$, a player $i$ selects the action $s_{i}\left(t_{i}\right)$ from the set $X_{i}$.

Let $\mathscr{P}_{i}$ denote the set of pure strategies for player $i$, and set $\mathscr{P}:=\times_{i=1}^{N} \mathscr{P}_{i}$.

\subsection{Behavioral strategies in Bayesian games}

Definition 4. Let $\Gamma=\left(\left(T_{i}, \mathscr{T}_{i}\right), X_{i}, u_{i}, p\right)_{i=1}^{N}$ be a Bayesian game. A behavioral strategy for player $i$ in $\Gamma$ is a transition probability with respect to $\left(T_{i}, \mathscr{T}_{i}\right)$ and $\left(X_{i}, \mathscr{B}\left(X_{i}\right)\right)$, i.e., a mapping

$$
\sigma_{i}: \mathscr{B}\left(X_{i}\right) \times T_{i} \rightarrow[0,1],
$$

where $\sigma_{i}\left(\cdot \mid t_{i}\right) \in \Delta\left(X_{i}\right)$ for each $t_{i} \in T_{i}$ and $\sigma_{i}(A \mid \cdot): T_{i} \rightarrow \mathbb{R}$ is a $\left(\mathscr{T}_{i}, \mathscr{B}(\mathbb{R})\right)$-measurable function for each $A \in \mathscr{B}\left(X_{i}\right)$.

Let $\mathscr{Y}_{i}$ represent the set of behavioral strategies for player $i$, and define $\mathscr{Y}:=\times_{i=1}^{N} \mathscr{Y}_{i}{ }^{4}$

Define the strategic-form game

$$
G^{b}:=\left(\mathscr{Y}_{i}, U_{i}\right)_{i=1}^{N}
$$

where $U_{i}: \mathscr{Y} \rightarrow \mathbb{R}$ is given by

$$
U_{i}\left(\sigma_{1}, \ldots, \sigma_{N}\right):=\int_{T} \int_{X_{N}} \cdots \int_{X_{1}} u_{i}(t, x) \sigma_{1}\left(d x_{1} \mid t_{1}\right) \cdots \sigma_{N}\left(d x_{N} \mid t_{N}\right) p(d t) .^{5}
$$

Every pure strategy in $\mathscr{P}_{i}$ induces a corresponding "pure" behavioral strategy in $\mathscr{Y}_{i}$ in a natural way. If $s_{i} \in \mathscr{P}_{i}$, define $\sigma_{i}^{s_{i}} \in \mathscr{Y}_{i}$ as follows: for $t_{i} \in T_{i}$ and $A \in \mathscr{B}\left(X_{i}\right)$,

$$
\sigma_{i}^{s_{i}}\left(A \mid t_{i}\right):=\delta_{s_{i}\left(t_{i}\right)}(A),
$$

where $\delta_{s_{i}\left(t_{i}\right)} \in \Delta\left(X_{i}\right)$ denotes the Dirac measure concentrated on the point $s_{i}\left(t_{i}\right) .^{6}$

If $s_{i} \in \mathscr{P}_{i}$ and $\sigma_{-i} \in \mathscr{Y}_{-i}$, then define

$$
U_{i}\left(s_{i}, \sigma_{-i}\right):=\int_{T} \int_{X_{N}} \cdots \int_{X_{1}} u_{i}\left(t,\left(s_{i}\left(t_{i}\right), x_{-i}\right)\right)\left[\prod_{j \neq i} \sigma_{j}\left(d x_{j} \mid t_{j}\right)\right] p(d t),
$$

and note that

$$
U_{i}\left(s_{i}, \sigma_{-i}\right)=U_{i}\left(\sigma_{i}^{s_{i}}, \sigma_{-i}\right) .
$$

Following Balder [6], we now describe the topological structure that we will impose on $\mathscr{Y}_{i}$. Define $\hat{\mathscr{L}}_{i}$ as the space of uniformly finite transition measures with respect to $\left(T_{i}, \mathscr{T}_{i}\right)$ and $\left(X_{i}, \mathscr{B}\left(X_{i}\right)\right.$. Recall that $\mathscr{C}\left(T_{i}, X_{i}\right)$ denotes the space of integrably bounded Carathéodory integrands on $T_{i} \times X_{i}$.

Definition 5. The narrow topology on $\hat{\mathscr{L}}_{i}$ is the weakest topology with respect to which all functionals in the set

$$
\left\{\varphi_{f}: f \in \mathscr{C}\left(T_{i}, X_{i}\right)\right\}
$$

are continuous, where $\varphi_{f}: \hat{\mathscr{L}}_{i} \rightarrow \mathbb{R}$ is defined for each $f \in \mathscr{C}\left(T_{i}, X_{i}\right)$ as

$$
\varphi_{f}(\mu):=\int_{T_{i}} \int_{X_{i}} f\left(t_{i}, x_{i}\right) \mu\left(d x_{i} \mid t_{i}\right) p_{i}\left(d t_{i}\right) .
$$

\footnotetext{
${ }^{4}$ We use $\mathscr{Y}$ as a mnemonic for Young measure and we reserve $\mathscr{B}$ for Borel sets.

5This definition of $U_{i}$ follows from Fubini's Theorem and the observation (see, e.g, Balder [6, p. 271]) that the product measure $\sigma_{1}\left(\cdot \mid t_{1}\right) \otimes \cdots \otimes \sigma_{N}\left(\cdot \mid t_{N}\right)$ defines a transition probability from $T$ into $\mathscr{B}\left(X_{1} \times \cdots \times X_{n}\right)=$ $\otimes_{i=1}^{N} \mathscr{B}\left(X_{i}\right)$.

${ }^{6}$ It can be shown that $\sigma_{i}^{s_{i}}(A \mid \cdot): T_{i} \rightarrow \mathbb{R}$ is $\left(\mathscr{T}_{i}, \mathscr{B}(\mathbb{R})\right)$-measurable for each $A \in \mathscr{B}\left(X_{i}\right)$.
} 
We view $\mathscr{Y}_{i}$ as a subspace of $\hat{\mathscr{L}}_{i}$ endowed with its relative topology. Theorem 2.2 in Balder [6] provides a useful characterization of the relative topology on $\mathscr{Y}_{i}$ that we use at several points in this paper. The Cartesian product $\mathscr{Y}$ is endowed with the corresponding product topology. By Theorem 2.3 in Balder [6], we have the following result.

Lemma 1. $\mathscr{Y}_{i}$ is a compact, convex subspace of the topological vector space $\hat{\mathscr{L}}_{i}$.

\section{Existence of behavioral strategy equilibrium: Uniform payoff se- curity}

The notion of behavioral strategy equilibrium employed in this paper is as follows.

Definition 6. A Bayes-Nash equilibrium of a Bayesian game $\Gamma=\left(\left(T_{i}, \mathscr{T}_{i}\right), X_{i}, u_{i}, p\right)_{i=1}^{N}$ is a Nash equilibrium of the game $G^{b}$ defined in (1), i.e., a profile $\left(\sigma_{1}, \ldots, \sigma_{N}\right) \in \mathscr{Y}$ such that for each $i$,

$$
U_{i}\left(\sigma_{i}, \sigma_{-i}\right) \geq U_{i}\left(v_{i}, \sigma_{-i}\right), \quad \text { for all } v_{i} \in \mathscr{Y}_{i}
$$

Next, we recall the notion of payoff security of Reny [60].

Definition 7 (Reny [60]). A topological game $\left(Z_{i}, g_{i}\right)_{i=1}^{N}$ is payoff secure if for each $\varepsilon>0$, $z \in \times{ }_{i=1}^{N} Z_{i}$, and $i$, there exists a $y_{i} \in Z_{i}$ and a neighborhood $V_{z_{-i}}$ of $z_{-i}$ such that $g_{i}\left(y_{i}, y_{-i}\right)>$ $g_{i}(z)-\varepsilon$ for every $y_{-i} \in V_{z_{-i}}$.

We next recall the notion of uniform payoff security for complete information games ( $f$. Monteiro and Page [51]), a condition on the primitives of a game that ensures that the game's mixed extension satisfies Reny's [60] payoff security (Definition 7). ${ }^{7}$

Definition 8 (Monteiro and Page [51]). A topological game $\left(Z_{i}, g_{i}\right)_{i \in N}$ is uniformly payoff secure if for each $i, \varepsilon>0$, and $z_{i} \in Z_{i}$, there exists $y_{i} \in Z_{i}$ such that for every $z_{-i} \in Z_{-i}$, there is a neighborhood $V_{z_{-i}}$ of $z_{-i}$ such that $g_{i}\left(y_{i}, y_{-i}\right)>g_{i}\left(z_{i}, z_{-i}\right)-\varepsilon$ for every $y_{-i} \in V_{z_{-i}}$.

We introduce the following extension of Definition 8 to the case of incomplete information games. This is a condition on the primitives of a Bayesian game ensuring that the strategic form game $G^{b}$ defined in (1) satisfies Reny's notion of payoff security provided above (see Lemma 2 below).

Definition 9. The Bayesian game $\left(\left(T_{i}, \mathscr{T}_{i}\right), X_{i}, u_{i}, p\right)_{i=1}^{N}$ is uniformly payoff secure if for each $i, \varepsilon>0$, and $s_{i} \in \mathscr{P}_{i}$, there exists $s_{i}^{*} \in \mathscr{P}_{i}$ such that for all $\left(t, x_{-i}\right) \in T \times X_{-i}$, there exists a neighborhood $V_{x_{-i}}$ of $x_{-i}$ such that

$$
u_{i}\left(t,\left(s_{i}^{*}\left(t_{i}\right), y_{-i}\right)\right)>u_{i}\left(t,\left(s_{i}\left(t_{i}\right), x_{-i}\right)\right)-\varepsilon, \quad \text { for all } y_{-i} \in V_{x_{-i}} \cdot 8
$$

The following condition implies uniform payoff security (Proposition 1 below) and proves useful in applications.

Condition 1. For each $i$ and $\varepsilon>0$, there exists a $\left(\mathscr{B}\left(X_{i}\right), \mathscr{B}\left(X_{i}\right)\right)$-measurable map $\phi: X_{i} \rightarrow X_{i}$ such that the following holds: for each $(t, x) \in T \times X$, there exists a neighborhood $V_{x_{-i}}$ of $x_{-i}$ such that

$$
u_{i}\left(t,\left(\phi\left(x_{i}\right), y_{-i}\right)\right)>u_{i}\left(t,\left(x_{i}, x_{-i}\right)\right)-\varepsilon, \quad \text { for all } y_{-i} \in V_{x_{-i}} .
$$

\footnotetext{
${ }^{7}$ The notion of uniform payoff security proves useful to establish existence of equilibrium in applied work (see, e.g., Carbonell-Nicolau and Ok [17, Lemmas 3 and 4]).

${ }^{8}$ All our results remain intact if "for all $(t, x) \in T \times X$ " in Definition 9 and Condition 1 is changed to "for all $(t, x) \in E \times X$, where $E \subseteq T$ and $\left(p_{1} \otimes \cdots \otimes p_{N}\right)(E)=1$."
} 
Remark 1. A natural extension of uniform payoff security to Bayesian games would simply require that each complete information game $G(t)=\left(X_{i}, u_{i}(t, \cdot)\right)_{i=1}^{N}$ associated with the Bayesian game $\Gamma=\left(\left(T_{i}, \mathscr{T}_{i}\right), X_{i}, u_{i}, p\right)_{i=1}^{N}$ satisfy the Monteiro-Page definition of uniform payoff security. Our notion of uniform payoff security for $G=\left(\left(T_{i}, \mathscr{T}_{i}\right), X_{i}, u_{i}, p\right)_{i=1}^{N}$ implies that, for each $t \in T$, the complete information game $G(t)=\left(X_{i}, u_{i}(t, \cdot)\right)_{i=1}^{N}$ satisfies the MonteiroPage definition. In the presence of infinite type sets however, we require that the actions $s_{i}^{*}\left(t_{i}\right)$ be "strung together" in a measurable fashion.

Proposition 1. Suppose that the Bayesian game $\Gamma=\left(\left(T_{i}, \mathscr{T}_{i}\right), X_{i}, u_{i}, p\right)_{i=1}^{N}$ satisfies Condition 1. Then $\Gamma$ is uniformly payoff secure.

Proof. Fix $i, \varepsilon>0$, and $s_{i} \in \mathscr{P}_{i}$, and let $\phi$ be given by Condition 1. Define $s_{i}^{*} \in \mathscr{P}_{i}$ as follows: $s_{i}^{*}\left(t_{i}\right):=\phi\left(s_{i}\left(t_{i}\right)\right)$. Given $\left(t, x_{-i}\right) \in T \times X_{-i}$, Condition 1 gives a neighborhood $V_{x_{-i}}$ of $x_{-i}$ such that

$$
u_{i}\left(t,\left(s_{i}^{*}\left(t_{i}\right), y_{-i}\right)\right)>u_{i}\left(t,\left(s_{i}\left(t_{i}\right), x_{-i}\right)\right)-\varepsilon, \quad \text { for all } y_{-i} \in V_{x_{-i}} .
$$

This completes the proof.

In the next lemmas, payoff security and upper semicontinuity in $G^{b}$ are defined with respect to the narrow topology. Lemma 2 generalizes Theorem 1 in Monteiro and Page [51].

Lemma 2. Suppose that the Bayesian game $\left(\left(T_{i}, \mathscr{T}_{i}\right), X_{i}, u_{i}, p\right)_{i=1}^{N}$ is uniformly payoff secure. If $p$ is absolutely continuous with respect to $p_{1} \otimes \cdots \otimes p_{N}$, then the game $G^{b}$ defined in (1) is payoff secure.

Lemma 3. Given a Bayesian game $\left(\left(T_{i}, \mathscr{T}_{i}\right), X_{i}, u_{i}, p\right)_{i=1}^{N}$, suppose that for each $t \in T$, the map $\sum_{i=1}^{N} u_{i}(t, \cdot): X \rightarrow \mathbb{R}$ is upper semicontinuous. Suppose further that $p$ is absolutely continuous with respect to $p_{1} \otimes \cdots \otimes p_{N}$. Then the map $\sum_{i=1}^{N} U_{i}(\cdot): \mathscr{Y} \rightarrow \mathbb{R}$ is upper semicontinuous.

The proofs of Lemma 2 and Lemma 3 are relegated to Section A.1.

Our first main existence result is Theorem 1.

Theorem 1. Suppose that the Bayesian game $\Gamma=\left(\left(T_{i}, \mathscr{T}_{i}\right), X_{i}, u_{i}, p\right)_{i=1}^{N}$ is uniformly payoff secure and that for each $t \in T$, the map $\sum_{i=1}^{N} u_{i}(t, \cdot): X \rightarrow \mathbb{R}$ is upper semicontinuous. If $p$ is absolutely continuous with respect to $p_{1} \otimes \cdots \otimes p_{N}$, then $G^{b}$ has a Nash equilibrium, i.e., a Bayes-Nash equilibrium of $\Gamma$.

Proof. For each $i, \mathscr{Y}_{i}$ is a compact, convex subspace of a topological vector space (Lemma 1 ), and for each $\sigma_{-i} \in \mathscr{Y}_{-i}$, the map $U_{i}\left(\cdot, \sigma_{-i}\right): \mathscr{Y}_{i} \rightarrow \mathbb{R}$ is quasiconcave. Hence, because the map $\sum_{i=1}^{N} U_{i}(\cdot): \mathscr{Y} \rightarrow \mathbb{R}$ is upper semicontinuous (Lemma 3), and since the game $G^{b}$ is payoff secure (Lemma 2), $G^{b}$ is better-reply secure (Reny [60], Proposition 3.2). Applying Theorem 3.1 of Reny [60] gives a Nash equilibrium of $G^{b}$, i.e., a Bayes-Nash equilibrium of $\Gamma$.

Theorem 1 and Proposition 1 immediately yield the following corollary.

Corollary 1. Suppose that the Bayesian game $\Gamma=\left(\left(T_{i}, \mathscr{T}_{i}\right), X_{i}, u_{i}, p\right)_{i=1}^{N}$ satisfies Condition 1 and that for each $t \in T$, the map $\sum_{i=1}^{N} u_{i}(t, \cdot): X \rightarrow \mathbb{R}$ is upper semicontinuous. If $p$ is absolutely continuous with respect to $p_{1} \otimes \cdots \otimes p_{N}$, then $\Gamma$ possesses a Bayes-Nash equilibrium. 


\section{Existence of behavioral strategy equilibrium: Uniform diagonal security}

In this section, we present an approach to equilibrium in discontinuous games of incomplete information using the Nikaido-Isoda aggregation function. Fundamental to this approach is the notion of diagonal transfer continuity of Baye et al. [13].

Definition 10 (Baye et al. [13]). A topological game $\left(Z_{i}, g_{i}\right)_{i=1}^{N}$ is diagonally transfer continuous if whenever $x \in Z$ is not a Nash equilibrium of $\left(Z_{i}, g_{i}\right)_{i=1}^{N}$, there exist $y \in Z$ and a neighborhood $V_{x}$ of $x$ such that

$$
\sum_{i=1}^{N} g_{i}\left(y_{i}, z_{-i}\right)-\sum_{i=1}^{N} g_{i}(z)>0, \quad \text { for all } z \in V_{x}
$$

We next recall the notion of uniform diagonal security for complete information games of Prokopovych and Yannelis [58], a condition on the primitives of a complete information game that implies diagonal transfer continuity (Definition 10) in the game's mixed extension.

Definition 11 (Prokopovych and Yannelis [58]). A topological game $\left(Z_{i}, g_{i}\right)_{i=1}^{N}$ is uniformly diagonally secure if for each $\varepsilon>0$ and $x \in Z$, there exists $x^{*} \in Z$ such that for all $y \in Z$, there exists a neighborhood $V_{y}$ of $y$ such that

$$
\sum_{i=1}^{N} g_{i}\left(x_{i}^{*}, z_{-i}\right)-\sum_{i=1}^{N} g_{i}(z)>\sum_{i=1}^{N} g_{i}\left(x_{i}, y_{-i}\right)-\sum_{i=1}^{N} g_{i}(y)-\varepsilon, \quad \text { for all } z \in V_{y} .
$$

We introduce the following extension of Definition 11 to the case of incomplete information games. This is a condition on the primitives of a Bayesian game ensuring that the strategic form game $G^{b}$ defined in (1) satisfies diagonal transfer continuity (see Lemma 4 below).

Definition 12. The Bayesian game $\left(\left(T_{i}, \mathscr{T}_{i}\right), X_{i}, u_{i}, p\right)_{i=1}^{N}$ is uniformly diagonally secure if for each $\varepsilon>0$ and $s \in \mathscr{P}$, there exists $s^{*} \in \mathscr{P}$ such that for all $(t, x) \in T \times X$, there exists a neighborhood $V_{x}$ of $x$ such that

$$
\sum_{i=1}^{N} u_{i}\left(t,\left(s_{i}^{*}\left(t_{i}\right), y_{-i}\right)\right)-\sum_{i=1}^{N} u_{i}(t, y)>\sum_{i=1}^{N} u_{i}\left(t,\left(s_{i}\left(t_{i}\right), x_{-i}\right)\right)-\sum_{i=1}^{N} u_{i}(t, x)-\varepsilon, \quad \text { for all } y \in V_{x} .
$$

The next condition provides an analogue of Condition 1 for uniform diagonal security.

Condition 2. For each $\varepsilon>0$ and $i$, there exists a $\left(\mathscr{B}\left(X_{i}\right), \mathscr{B}\left(X_{i}\right)\right)$-measurable map $\phi_{i}: X_{i} \rightarrow$ $X_{i}$ such that the following holds: for each $(t, x, y) \in T \times X \times X$, there exists a neighborhood $V_{x}$ of $x$ such that

$$
\sum_{i=1}^{N} u_{i}\left(t,\left(\phi_{i}\left(y_{i}\right), z_{-i}\right)\right)-\sum_{i=1}^{N} u_{i}(t, z)>\sum_{i=1}^{N} u_{i}\left(t,\left(y_{i}, x_{-i}\right)\right)-\sum_{i=1}^{N} u_{i}(t, x)-\varepsilon, \quad \text { for all } z \in V_{x} .
$$

Proposition 2. Suppose that the Bayesian game $\Gamma=\left(\left(T_{i}, \mathscr{T}_{i}\right), X_{i}, u_{i}, p\right)_{i=1}^{N}$ satisfies Condition 2. Then $\Gamma$ is uniformly diagonally secure.

Proof. Fix $\varepsilon>0$ and $s \in \mathscr{P}$, and for each $i$ let $\phi_{i}$ be given by Condition 2. Define $s^{*} \in \mathscr{P}$ as follows: for each $i, s_{i}^{*}\left(t_{i}\right):=\phi_{i}\left(s_{i}\left(t_{i}\right)\right)$. Given $(t, x) \in T \times X$, Condition 2 gives a neighborhood $V_{x}$ of $x$ such that

$$
\sum_{i=1}^{N} u_{i}\left(t,\left(\phi_{i}\left(s_{i}\left(t_{i}\right)\right), z_{-i}\right)\right)-\sum_{i=1}^{N} u_{i}(t, z)>\sum_{i=1}^{N} u_{i}\left(t,\left(s_{i}\left(t_{i}\right), x_{-i}\right)\right)-\sum_{i=1}^{N} u_{i}(t, x)-\varepsilon, \quad \text { for all } z \in V_{x} .
$$


Therefore,

$$
\sum_{i=1}^{N} u_{i}\left(t,\left(s_{i}^{*}\left(t_{i}\right), z_{-i}\right)\right)-\sum_{i=1}^{N} u_{i}(t, z)>\sum_{i=1}^{N} u_{i}\left(t,\left(s_{i}\left(t_{i}\right), x_{-i}\right)\right)-\sum_{i=1}^{N} u_{i}(t, x)-\varepsilon, \quad \text { for all } z \in V_{x},
$$

and so $\Gamma$ is uniformly diagonally secure.

The proof of the following lemma is relegated to Section A.1.

Lemma 4. Suppose that the Bayesian game $\left(\left(T_{i}, \mathscr{T}_{i}\right), X_{i}, u_{i}, p\right)_{i=1}^{N}$ is uniformly diagonally secure. If $p$ is absolutely continuous with respect to $p_{1} \otimes \cdots \otimes p_{N}$, then the game $G^{b}$ defined in (1) is diagonally transfer continuous.

We now present our second main existence result in terms of uniform diagonal security.

Theorem 2. Suppose that the Bayesian game $\Gamma=\left(\left(T_{i}, \mathscr{T}_{i}\right), X_{i}, u_{i}, p\right)_{i=1}^{N}$ is uniformly diagonally secure. If $p$ is absolutely continuous with respect to $p_{1} \otimes \cdots \otimes p_{N}$, then $G^{b}$ has a Nash equilibrium, i.e., a Bayes-Nash equilibrium of $\Gamma$.

Proof. Applying Lemma 2, it follows that $G^{b}$ is diagonally transfer continuous. As remarked in Prokopovych and Yannelis [58], the mapping $F: \mathscr{Y} \times \mathscr{Y} \rightarrow \mathbb{R}$ defined as

$$
F(v, \sigma)=\sum_{i=1}^{N} U_{i}\left(v_{i}, \sigma_{-i}\right)-\sum_{i=1}^{N} U_{i}(\sigma)
$$

satisfies the definition of 0-transfer lower semicontinuity in $\sigma$ (see [58] or Nessah and Tian [53]). Combining this observation with Proposition 2.1 and Remark 2.3 in Nessah and Tian [53] and applying Theorem 3.1 in their paper, we conclude that $G^{b}$ has a Nash equilibrium, i.e., a Bayes-Nash equilibrium of $\Gamma$.

Theorem 2 and Proposition 2 immediately yield the following corollary.

Corollary 2. Suppose that the Bayesian game $\Gamma=\left(\left(T_{i}, \mathscr{T}_{i}\right), X_{i}, u_{i}, p\right)_{i=1}^{N}$ satisfies Condition 2. If $p$ is absolutely continuous with respect to $p_{1} \otimes \cdots \otimes p_{N}$, then $\Gamma$ possesses a Bayes-Nash equilibrium.

\section{Discussion}

\subsection{Related literature}

In the seminal paper of Milgrom and Weber [50], it is assumed that the players' type spaces and action spaces are compact metric spaces and that a player's payoff is jointly continuous in type-action profiles. In Balder [6], the existence result of Milgrom and Weber [50] is extended to the case in which type spaces need only be measurable spaces and payoff functions are jointly measurable in type-action profiles and continuous in action profiles. In Balder [9], this result is generalized to the case in which players' strategy sets are completely regular Souslin spaces. In Balder [11], the existence result in Balder [6] is extended to the case of countably many players (cf. Balder [11], Theorem 3.4.1). In Balder and Rustichini [8], a further generalization to the case of uncountably many players is presented. In a recent paper, He and Yannelis [35] conduct an analysis similar to ours, based on the notion of disjoint payoff matching ( $c f$. Allison and Lepore [2]), which is extended to the case of incomplete information.

When the private information of a player is represented by a set $T_{i}$, a strategy is a function that maps $T_{i}$ into actions. As an alternative to the types representation of private 
information, one can begin with a measurable state space $(\Omega, \mathscr{F})$ and model the private information of a player $i$ as a sub-sigma field $\mathscr{A}_{i}$ of $\mathscr{F}$. In this framework, a strategy is an $\mathscr{A}_{i}$-measurable map from $\Omega$ into actions. Yannelis and Rustichini [79] prove the existence of Bayes-Nash equilibrium in the state-space setup assuming that the state space is a measurable space, payoffs are jointly measurable in state-action profiles, and continuous in action profiles. In a state space model with countably many states, He and Yannelis [34] provide existence results for discontinuous payoffs satisfying two variations of Monteiro and Page's [51] uniform payoff security.

In a model with infinitely many players, Balder [7] proves the existence of an equilibrium in a state-space framework in which payoffs are jointly measurable in state-action profiles, upper semicontinuous in own actions and continuous in aggregate profiles of transition probabilities ( $c f$. Balder [7], Proposition 2). For the class of affine games with indeterminate outcomes, Jackson et al. [38] prove the existence of an equilibrium in a communication extension of a game with incomplete information that exhibits certain discontinuities. In their setup, type spaces are compact metric and payoffs are continuous in type profiles. By restricting attention to elementary communication devices, Balder [12] extends the analysis in Jackson et al. [38] to the case in which type spaces are measurable spaces.

\subsection{Existence of equilibrium in distributional strategies}

Definition 13. Let $\Gamma=\left(\left(T_{i}, \mathscr{T}_{i}\right), X_{i}, u_{i}, p\right)_{i=1}^{N}$ be a Bayesian game. Adistributional strategy for player $i$ in $\Gamma$ is a probability measure $\mu_{i}$ on $\left(T_{i} \times X_{i}, \mathscr{T}_{i} \otimes \mathscr{B}\left(X_{i}\right)\right)$ such that

$$
\mu_{i}\left(A \times X_{i}\right)=p_{i}(A), \quad \text { for all } A \in \mathscr{T}_{i}
$$

Let $\mathscr{D}_{i}$ represent the set of distributional strategies for player $i$, and define $\mathscr{D}:=\times_{i=1}^{N} \mathscr{D}_{i}$. Given $\mu_{i} \in \mathscr{D}_{i}$, the map $t_{i} \in T_{i} \mapsto \mu_{i}\left(\cdot \mid t_{i}\right)$ will denote a corresponding version of the regular conditional probability measure on $X_{i}$.

Define the strategic-form game

$$
G^{d}:=\left(\mathscr{D}_{i}, \Phi_{i}\right)_{i=1}^{N}
$$

where $\Phi_{i}: \mathscr{D} \rightarrow \mathbb{R}$ by

$$
\Phi_{i}\left(\mu_{1}, \ldots, \mu_{N}\right):=\int_{T} \int_{X_{N}} \cdots \int_{X_{1}} u_{i}(t, x) \mu_{1}\left(d x_{1} \mid t_{1}\right) \cdots \mu_{N}\left(d x_{N} \mid t_{N}\right) p(d t) .
$$

Remark 2. As remarked in Milgrom and Weber [50], every behavioral strategy gives rise to a natural distributional strategy, and every distributional strategy $\mu_{i}$ corresponds to a class of behavioral strategies defined as regular conditional probabilities induced by $\mu_{i}$. Regular conditional probability measures exist in our framework as a consequence of, e.g., Theorem 10.2.2 in Dudley [25].

It is straightforward to see that a Nash equilibrium $\left(\sigma_{1}, \ldots, \sigma_{N}\right) \in \mathscr{Y}$ of the game $G^{b}$ defined in (1) induces a Nash equilibrium $\left(\mu_{1}, \ldots, \mu_{N}\right) \in \mathscr{D}$ of the game $G^{d}$ defined in (2), where for each $i, \mu_{i}$ is defined by

$$
\mu_{i}(S \times A):=\int_{S} \sigma_{i}\left(A \mid t_{i}\right) p_{i}\left(d t_{i}\right) .
$$

Consequently, $G^{d}$ has a Nash equilibrium if $G^{b}$ has a Nash equilibrium. Conversely, given a Nash equilibrium $\left(\mu_{1}, \ldots, \mu_{N}\right) \in \mathscr{D}$ of $G^{d}$, a corresponding vector of regular conditional probability measures, $\left(t_{1}, . ., t_{N}\right) \mapsto\left(\mu_{1}\left(\cdot \mid t_{1}\right), \ldots, \mu_{N}\left(\cdot \mid t_{N}\right)\right)$, viewed as a member of $\mathscr{Y}$, is a Nash equilibrium in $G^{b}$. 
While Theorem 1 already implies the existence of a Nash equilibrium in $G^{d}$ when each $\mathscr{Y}_{i}$ is endowed with the narrow topology, the existence of Nash equilibria in $G^{d}$ can be established directly if we endow the strategy sets $\mathscr{D}_{i}$ with an appropriate topology and show that our conditions on the primitives of a Bayesian game imply payoff security and upper semicontinuity of the sum $\sum_{i=1}^{N} \Phi_{i}$ in the game $G^{d}$ given our choice of a topology on $\mathscr{D}_{i}$. To sketch this alternative approach, let $\mathscr{L}_{i}$ be the set of all finite signed measures defined on the measurable space $\left(T_{i} \times X_{i}, \mathscr{T}_{i} \otimes \mathscr{B}\left(X_{i}\right)\right)$. Recall that $\mathscr{C}\left(T_{i}, X_{i}\right)$ denotes the space of integrably bounded Carathéodory integrands on $T_{i} \times X_{i}$.

Definition 14. The ws-topology (weak-strong topology) on $\mathscr{L}_{i}$ is the weakest topology for which all functionals in

$$
\left\{\psi_{f}: f \in \mathscr{C}\left(T_{i}, X_{i}\right)\right\}
$$

are continuous, where $\psi_{f}: \mathscr{L}_{i} \rightarrow \mathbb{R}$ is defined for each $f \in \mathscr{C}\left(T_{i}, X_{i}\right)$ as

$$
\psi_{f}(\mu):=\int_{T_{i} \times X_{i}} f\left(t_{i}, x_{i}\right) \mu\left(d t_{i}, d x_{i}\right) .^{9}
$$

We endow $\mathscr{L}_{i}$ with the ws-topology and view $\mathscr{D}_{i}$ as a subspace of $\mathscr{L}_{i}$ with the relative topology. The Cartesian product $\mathscr{D}$ is endowed with the corresponding product topology. Since $\mathscr{L}_{i}$ is a vector space for the usual addition and scalar multiplication of measures and since the ws-topology is the initial topology generated by a collection of linear functions on $\mathscr{L}_{i}$, it follows that $\mathscr{L}_{i}$ is a topological vector space with respect to the ws-topology (e.g., see Horvath [36, Chapter 2, Section 11]). If $\Gamma=\left(\left(T_{i}, \mathscr{T}_{i}\right), X_{i}, u_{i}, p\right)_{i=1}^{N}$ is a Bayesian game, then it can be shown that (i) $\mathscr{D}_{i}$ is a compact, convex subset of $\mathscr{L}_{i}$ and (ii) if $\Gamma$ is uniformly payoff secure and $p$ is absolutely continuous with respect to $p_{1} \otimes \cdots \otimes p_{N}$, then the game $G^{d}$ is payoff secure with respect to the ws-topology. To sketch the arguments for these observations, let $\hat{\mathscr{L}}_{i}$ be the space defined in Subsection 2.2 and, following Balder [6], define $\hat{\mathscr{N}}_{i}$ as the subspace of $\hat{\mathscr{L}}_{i}$ defined as

$$
\hat{\mathscr{N}}_{i}:=\left\{\mu \in \hat{\mathscr{L}}_{i}: \int_{T_{i}} \int_{X_{i}} f\left(t_{i}, x_{i}\right) \mu\left(d x_{i} \mid t_{i}\right) p_{i}\left(d t_{i}\right)=0, \text { for all } f \in \mathscr{C}\left(\mathscr{T}_{i}, X_{i}\right)\right\} .
$$

Then $\hat{\mathscr{N}}_{i}$ is a closed linear subspace of $\hat{\mathscr{L}}_{i}$ in the narrow topology and the quotient mapping $\pi_{i}: \hat{\mathscr{L}}_{i} \rightarrow \hat{\mathscr{L}}_{i} / \hat{\mathscr{N}}_{i}$ is defined as

$$
\pi_{i}(\mu)=\left\{\mu+v: v \in \hat{\mathscr{N}}_{i}\right\}
$$

The quotient space $\hat{\mathscr{L}}_{i} / \hat{\mathscr{N}}_{i}$, endowed with the narrow quotient topology is a Hausdorff, locally convex topological vector space (Balder [6, p. 268]). Since the quotient map $\pi_{i}: \hat{\mathscr{L}}_{i} \rightarrow \hat{\mathscr{L}}_{i} / \hat{\mathscr{N}}_{i}$ is continuous, it follows that $\pi_{i}\left(\mathscr{Y}_{i}\right)$ is compact in $\hat{\mathscr{L}}_{i} / \hat{\mathscr{N}}_{i}$ as a consequence of Lemma 1 . As we discussed above, every member of $\mathscr{D}_{i}$ corresponds to an equivalence class of regular conditional probabilities, i.e., a member of $\pi_{i}\left(\mathscr{Y}_{i}\right)$. Conversely, a member of $\pi_{i}\left(\mathscr{Y}_{i}\right)$ induces an element of $\mathscr{D}_{i}$ in the obvious way. Combining Theorem 2.2 in Balder [6], Theorem 2.1.3 in Castaing et al. [20] and the remark in Balder [10, p. 497], it follows that the spaces $\mathscr{D}_{i}$ and $\pi_{i}\left(\mathscr{Y}_{i}\right)$ are homeomorphic when $\mathscr{D}_{i}$ is endowed with the ws-topology and $\pi_{i}\left(\mathscr{Y}_{i}\right)$ is endowed with the (relative) quotient narrow topology. Consequently, $\mathscr{D}$ and $\times_{i=1}^{N} \pi_{i}\left(\mathscr{Y}_{i}\right)$ are homeomorphic with respect to their associated product topologies. Note that $\mathscr{D}_{i}$ is obviously convex and, denoting the homeomorphism of $\mathscr{D}_{i}$ onto $\pi_{i}\left(Y_{i}\right)$ as $h_{i}$, it follows that $\mathscr{D}_{i}=h_{i}^{-1}\left(\pi_{i}\left(Y_{i}\right)\right)$ is compact since $\pi_{i}\left(Y_{i}\right)$ is compact, so (i) is proved. To prove (ii), we must show that $\left(\mathscr{D}_{i}, \Phi_{i}\right)_{i=1}^{N}$ is payoff secure with respect to the ws-topology and this can be shown using the observations that the spaces $\mathscr{D}_{i}$ and $\pi_{i}\left(\mathscr{Y}_{i}\right)$ are homeomorphic

\footnotetext{
${ }^{9}$ The ws-topology on a set of finite, nonnegative measures was introduced by Schäl [68].
} 
and that the game $\left(\mathscr{Y}_{i}, U_{i}\right)_{i=1}^{N}$ is payoff secure when each $\mathscr{Y}_{i}$ is endowed with the narrow topology (Lemma 2). Using an argument analogous to the proof of Lemma 3, it follows that the map $\sum_{i=1}^{N} \Phi_{i}(\cdot): \mathscr{D} \rightarrow \mathbb{R}$ is upper semicontinuous with respect to the ws-topology if $\sum_{i=1}^{N} u_{i}(t, \cdot): X \rightarrow \mathbb{R}$ is upper semicontinuous and we obtain the following complement to Theorem 1.

Theorem 3. Suppose that the Bayesian game $\Gamma=\left(\left(T_{i}, \mathscr{T}_{i}\right), X_{i}, u_{i}, p\right)_{i=1}^{N}$ is uniformly payoff secure and that for each $t \in T$, the map $\sum_{i=1}^{N} u_{i}(t, \cdot): X \rightarrow \mathbb{R}$ is upper semicontinuous. If $p$ is absolutely continuous with respect to $p_{1} \otimes \cdots \otimes p_{N}$ and each $\mathscr{D}_{i}$ is endowed with the (relative) ws-topology, then $G^{d}$ has a Nash equilibrium, i.e., a Bayes-Nash equilibrium of $\Gamma$.

\section{Applications}

We illustrate our existence results in several discontinuous economic games. It is the presence of these discontinuities that precludes the application of the existence results in Milgrom and Weber [50] and Balder [6]. ${ }^{10}$

\subsection{Equilibrium existence in common value auctions}

In this section we establish existence of equilibrium in a (single unit) common values auction setting. Our model encompasses, for example, all pay auctions and the war of attrition. ${ }^{11,12}$ We establish existence of equilibrium in behavioral (or distributional) strategies (as opposed to pure strategies), which is all one can hope to obtain given the generality of the games considered. In fact, not all auctions in our setting have pure strategy equilibria.

Recent work on the existence of equilibrium in auctions can be found in Krishna and Morgan [40], Lebrun [41], Reny [60], Lizzeri and Persico [45], Maskin and Riley [47], Athey [5], Reny and Zamir [62], Jackson and Swinkels [39], Monteiro and Moreira [52], Araujo et al. [3], and Araujo and de Castro [4]. Of these papers, Krishna and Morgan [40], Lebrun [41], Reny [60], Lizzeri and Persico [45], Maskin and Riley [47], Athey [5], Reny and Zamir [62], Araujo et al. [3], and Araujo and de Castro [4] confine attention to either independent or affiliated types ( $c f$. Milgrom and Weber [49]), while we do not impose any constraints (beyond the absolutely continuous information assumption ( $c f$. Assumption A below)) on the correlation of the players' types. While affiliation of types has proven useful in the study of auction games, de Castro [21] pinpoints its limitations and emphasizes the importance of relaxing the affiliation assumption. Jackson and Swinkels [39] and Monteiro and Moreira [52] allow for nonaffiliated types, but they confine attention (unlike the analysis in this section) to private values. ${ }^{13}$

There are $N$ bidders competing for an object. After learning her type, player $i$ submits a sealed bid $b_{i}$ from a closed and bounded interval $B_{i}$ in $\mathbb{R}_{+}$. Each $B_{i}$ is endowed with the usual relative Euclidean metric, and the Cartesian product $B:=\times_{i=1}^{N} B_{i}$ is equipped with the corresponding supremum metric. Let $T_{1}, \ldots, T_{N}$ be the type spaces (each $T_{i}$ is an arbitrary, nonempty type space with associated $\sigma$-algebra $\mathscr{T}_{i}$ ). If player $i$ wins the object when Nature chooses a type profile $t=\left(t_{1}, \ldots, t_{N}\right) \in T$ and when the profile of bids chosen by the players is

\footnotetext{
${ }^{10}$ Athey [5], McAdams [48], and Reny [61] have identified conditions, including continuity assumptions that we do not make here, under which pure-strategy monotone equilibria exist.

${ }^{11}$ The situations covered here go beyond the strict auction setting. In fact, all pay auctions are widely used to model contests, including technological competition and R\&D races, political contests, rent-seeking and lobbying activities, job promotion tournaments, and competition for a monopoly position; and the war of attrition has been used to model conflict among animals and survival among firms.

${ }^{12}$ We also cover oligopolistic competition à la Bertrand with symmetric cost functions. See Remark 6 .

${ }^{13}$ As illustrated in Jackson [37], the existence of equilibrium in a general auction setting with both common and private components to bidders' valuations is a delicate matter.
} 
$b=\left(b_{1}, \ldots, b_{N}\right) \in \times_{i=1}^{N} B_{i}$, then player $i$ 's payoff is given by $f_{i}(t, b)+h_{i}(t, b)$. All other bidders $j \neq i$ receive a payoff of $g_{j}(t, b)+h_{j}(t, b)$. The highest bidder wins the object and ties are broken via an equal probability rule. The common prior over type profiles in $T$ is represented by a probability measure $p$ on $\left(T, \otimes_{i} \mathscr{T}_{i}\right)$.

Bidder $i$ 's expected payoff at $t=\left(t_{1}, \ldots, t_{N}\right) \in T$ and $b=\left(b_{1}, \ldots, b_{N}\right) \in B$ is given by

$$
u_{i}(t, b):= \begin{cases}g_{i}(t, b)+h_{i}(t, b) & \text { if } b_{i}<\max _{j} b_{j}, \\ \frac{f_{i}(t, b)}{\#\left\{j: b_{j}=\max _{\iota} b_{l}\right\}}+\left(1-\frac{1}{\#\left\{j: b_{j}=\max _{\iota} b_{l}\right\}}\right) g_{i}(t, b)+h_{i}(t, b) & \text { if } b_{i}=\max _{j} b_{j} .\end{cases}
$$

Here, for each $i, f_{i}: T \times B \rightarrow \mathbb{R}, g_{i}: T \times B \rightarrow \mathbb{R}$, and $h_{i}: T \times B \rightarrow \mathbb{R}$ are assumed bounded and $\left(\left[\otimes_{j=1}^{N} \mathscr{T}_{j}\right] \otimes\left[\otimes_{j=1}^{N} \mathscr{B}\left(B_{j}\right)\right], \mathscr{B}(\mathbb{R})\right)$-measurable maps.

The associated Bayesian game is

$$
\Gamma:=\left(\left(T_{i}, \mathscr{T}_{i}\right), B_{i}, u_{i}, p\right)_{i=1}^{N}
$$

We make the following assumptions:

Assumption A. $p$ is absolutely continuous with respect to $p_{1} \otimes \cdots \otimes p_{N}$.

Assumption B. $B_{1}=\cdots=B_{N}=[\underline{b}, \bar{b}]$.

Assumption C. For each $i, f_{i}, g_{i}$, and $h_{i}$ satisfy the following:

(i) For each $i$, the families $\left\{f_{i}(t, \cdot): t \in T\right\},\left\{g_{i}(t, \cdot): t \in T\right\}$, and $\left\{h_{i}(t, \cdot): t \in T\right\}$ are equicontinuous on $B$.

(ii) For each $i$ and $\left(t_{i}, b_{i}\right) \in T_{i} \times B_{i}$, the following holds: if $f_{i}\left(\left(t_{i}, t_{-i}\right),\left(b_{i}, b_{-i}\right)\right)<g_{i}\left(\left(t_{i}, t_{-i}\right),\left(b_{i}, b_{-i}\right)\right)$ for some $\left(t_{-i}, b_{-i}\right) \in T_{-i} \times B_{-i}$, then $f_{i}\left(\left(t_{i}, t_{-i}^{\prime}\right),\left(b_{i}, b_{-i}^{\prime}\right)\right)<g_{i}\left(\left(t_{i}, t_{-i}^{\prime}\right),\left(b_{i}, b_{-i}^{\prime}\right)\right)$ for every $\left(t_{-i}^{\prime}, b_{-i}^{\prime}\right) \in T_{-i} \times B_{-i}$.

Remark 3. Because $B$ is compact, it follows from Assumption C(i) that the families $\left\{f_{i}(t, \cdot)\right.$ : $t \in T\},\left\{g_{i}(t, \cdot): t \in T\right\}$, and $\left\{h_{i}(t, \cdot): t \in T\right\}$ are uniformly equicontinuous on $B$.

Remark 4. Assumption C(ii) is innocuous in the cases of all pay auctions and the war of attrition, since for these game forms it is commonly assumed that $f_{i} \geq g_{i}$ for each $i$. Without Assumption C(ii), particular instances of the game defined in (4) can be found that violate uniform payoff security. Thus, Assumption C(ii) is needed to apply the abstract existence results developed in Section 3.

Remark 5. Assumption C(i) is used in the proof of Corollary 3 to establish uniform payoff security of the Bayesian game $\Gamma$. The order of quantifiers in the definition of uniform payoff security (Definition 9), together with the argument used in the proof of Corollary 3 to prove uniform payoff security of $\Gamma$, suggests that a weakening of Assumption C(i) would likely be enough to prove Corollary 3 , at the cost of a more involved construction of the strategy $s_{i}^{*}\left(t_{i}\right)$. To keep the illustration of our general existence results simple, we do not pursue this exercise here.

Assumption D (Common values). $f_{1}=\cdots=f_{N}=: f$ and $g_{1}=\cdots=g_{N}=: g .{ }^{14}$

Corollary 3 (to Theorem 1). Under Assumptions A-D, the auction game $\Gamma$ defined in (4) possesses a Bayes-Nash equilibrium.

\footnotetext{
${ }^{14}$ In the common values setting, the maps $h_{i}$ allow for a certain form of asymmetry across players.
} 
The proof of Corollary 3 is presented in Subsection A.2.1 of the Appendix.

Remark 6. An existence result analogous to Corollary 3 can be derived (under Assumptions A-D) for the following modification of the game in (4):

$$
\Gamma^{*}:=\left(\left(T_{i}, \mathscr{T}_{i}\right), B_{i}, u_{i}^{*}, p\right)_{i=1}^{N},
$$

where

$$
u_{i}^{*}(t, b):= \begin{cases}g_{i}(t, b)+h_{i}(t, b) & \text { if } b_{i}>\min _{j} b_{j}, \\ \frac{f_{i}(t, b)}{\#\left\{j: b_{j}=\min _{\iota} b_{\iota}\right\}}+\left(1-\frac{1}{\#\left\{j: b_{j}=\min _{\iota} b_{\iota}\right\}}\right) g_{i}(t, b)+h_{i}(t, b) & \text { if } b_{i}=\min _{j} b_{j} .\end{cases}
$$

Under Assumptions A-D, the game defined in (5) can be viewed as a game of Bertrand competition with symmetric costs. To see this, it suffices to set each $h_{i}$ equal to zero and define $f_{i}(t, b)$ as the profit of a monopolist $i$ at price $b_{i}$ given a type profile $t \in T .{ }^{15}$ Observe that implicit in this interpretation of (5) is the assumption that individual cost functions are identical across firms.

\subsection{Equilibrium existence in Cournot games}

The role of incomplete information in Cournot oligopolies (and in particular the value of information and the incentives for firms to share information) has been studied extensively (see, e.g., Novshek and Sonnenschein [56], Clarke [22], Vives [75, 76], Gal-Or [32, 33], Sakai [66, 67], Shapiro [69], Raith [59], and Einy et al. [28, 29, 30]). The relevant literature focuses on pure-strategy equilibria and circumvents the issue of equilibrium existence by making strong assumptions. For example, Novshek and Sonnenschein [56], Clarke [22], Vives [76, 75], Gal-Or [32, 33], Sakai [66, 67], Shapiro [69], and Raith [59] confine attention to either linear demand or linear costs, Einy et al. [28] posit the existence of an equilibrium and investigate its properties, and Einy et al. [29] assume that firms are symmetrically informed. In Einy et al. [30] it is shown that when firms have incomplete information about market demand and cost functions, a Cournot equilibrium in pure strategies need not exist, even in simple cases with linear demand and cost functions. The existence of equilibrium in behavioral (or distributional) strategies easily follows from standard arguments if market demand and cost functions are assumed continuous, but remains an open question in the presence of discontinuities. In this section we prove an existence result for Cournot oligopolies with incomplete information and cost discontinuities. By allowing for cost discontinuities, we cover the case of nonsunk fixed costs (cf. Daughety [26], p. 2) as well as other economic phenomena leading to these kinds of discontinuities, including inflexibility in hiring decisions as a result of collective bargaining agreements, imposition of pollution abatement taxes for production beyond a certain scale, lumpiness in production, and congestion effects ( $c f$. Brems [16], Friedman [31], and Baye and Morgan [14]).

Consider a market for a single homogeneous good in which $N$ firms compete in quantities. Let $T_{1}, \ldots, T_{N}$ be the firms' type spaces (each $T_{i}$ is an arbitrary, nonempty type space with associated $\sigma$-algebra $\left.\mathscr{T}_{i}\right)$. Given a type profile $t=\left(t_{1}, \ldots, t_{N}\right) \in T$, where $t_{i}$ represents firm $i$ 's type, the market's inverse demand function is given by $p(t, \cdot)$. Thus, $p(t, q)$ represents the price that clears the market in state $t$ when aggregate output is $q$. Each firm $i \in\{1, \ldots, N\}$ faces a cost function $c_{i}\left(t, q_{i}\right)$ defined on type profiles $t$ and individual output levels $q_{i}$ selected from a compact subset $X_{i}$ of $\mathbb{R}_{+}$. The common prior over type profiles in $T$ is denoted $\eta$ (a probability measure over $\left(T, \otimes_{i} \mathscr{T}_{i}\right)$ ), with corresponding marginal probability measures $\eta_{1}, \ldots, \eta_{N}$

\footnotetext{
${ }^{15}$ If $f_{i}$ represents long run profit, then $f_{i} \geq 0$, and in this case one defines $g_{i}:=0$.
} 
This model can be formally described as a Bayesian game

$$
\Gamma:=\left(\left(T_{i}, \mathscr{T}_{i}\right), X_{i}, u_{i}, \eta\right)_{i=1}^{N}
$$

where, for each $i$,

$$
u_{i}\left(t,\left(q_{1}, \ldots, q_{N}\right)\right):=q_{i} p\left(t, \sum_{j=1}^{N} q_{j}\right)-c_{i}\left(t, q_{i}\right),
$$

where $p: T \times \mathbb{R}_{+} \rightarrow \mathbb{R}_{+}$and $c_{i}: T \times \mathbb{R}_{+} \rightarrow \mathbb{R}_{+}$.

We make the following assumptions:

Assumption E. $\eta$ is absolutely continuous with respect to $\eta_{1} \otimes \cdots \otimes \eta_{N}$.

Assumption F. The maps $p: T \times \mathbb{R}_{+} \rightarrow \mathbb{R}_{+}$and $c_{i}: T \times \mathbb{R}_{+} \rightarrow \mathbb{R}_{+}$are $\left(\left[\otimes_{i=1}^{N} \mathscr{T}_{i}\right] \otimes \mathscr{B}\left(\mathbb{R}_{+}\right), \mathscr{B}(\mathbb{R})\right)$ measurable and bounded.

Assumption G. The map $\left.p(t, \cdot)\right|_{\left\{\sum_{i=1}^{N} q_{i}:\left(q_{1}, \ldots, q_{N}\right) \in X\right\}}$ is continuous for each $t \in T$.

Assumption H. For each $t \in T$, the map $\left[q \mapsto \sum_{i=1}^{N} c_{i}\left(t, q_{i}\right)\right]: X \rightarrow \mathbb{R}$ is lower semicontinuous.

Corollary 4 (to Theorem 1). Under Assumptions E-H, the Cournot game $\Gamma$ defined in (6) possesses a Bayes-Nash equilibrium.

The proof of Corollary 4 is relegated to Subsection A.2.2 of the Appendix.

Remark 7. The following two conditions weaken Assumptions G-H combined and, together with Assumption E, are sufficient for the conclusion of Corollary 4: (i) the map $\left.p(t, \cdot)\right|_{\left\{\sum_{i=1}^{N} q_{i}:\left(q_{1}, \ldots, q_{N}\right) \in X\right\}}$ is lower semicontinuous for each $t \in T$; and (ii) the map

$$
\left[q \mapsto\left(\sum_{i=1}^{N} q_{i}\right) p\left(t, \sum_{i=1}^{N} q_{i}\right)-\left(\sum_{i=1}^{N} c_{i}\left(t, q_{i}\right)\right)\right]: X \rightarrow \mathbb{R}
$$

is upper semicontinuous for each $t \in T$.

\subsection{Equilibrium existence in Bertrand-Edgeworth games}

Economists have long recognized the importance of studying oligopoly games in which both price and quantity (rather than just price or quantity) are decision variables. This idea goes back to Edgeworth [27], who pointed out that firms may be unable or unwilling to supply all the forthcoming demand at the set prices, and Shubik [70], who advocated for "price-quantity" games in which sellers simultaneously post prices and quantities. On the other hand, there is a large literature on oligopoly theory with incomplete information, essentially in the form of cost and/or demand uncertainty, that studies the incentives of firms to share information, the welfare consequences of strategic information revelation, limit pricing, and information manipulation ( $c f$. Vives [77] and references therein). While extant work on Bertrand-Edgework competition with incomplete information (see, e.g., Staiger and Wolak [72], Reynolds and Wilson [63], and Lepore [42, 43]) restricts attention to games in which firms first choose production capacities and then compete in prices, the case of simultaneous selection of price-quantity pairs is not covered. In this section, we establish existence of equilibrium in a strategic-form Bertrand-Edgeworth game with demand and/or cost uncertainty and cost discontinuities. We cover the case of production in advance (as opposed to production to order) and extend Theorem 1 in Dasgupta and Maskin [24] and Theorem 1 in Maskin [46], which do not consider incomplete information or cost 
discontinuities (e.g., the case of nonsunk fixed costs and a variety of economic phenomena leading to this kind of discontinuities, as documented in Baye and Morgan [14]).

To simplify the exposition, we focus on the case of a duopoly, but the analysis extends to the $N$-firm case. There are two producers of a homogeneous good. Let $T_{1}$ and $T_{2}$ be the firms' type spaces (each $T_{i}$ is an arbitrary, nonempty type space with associated $\sigma$-algebra $\left.\mathscr{T}_{i}\right)$. Given a type profile $t=\left(t_{1}, t_{2}\right) \in T$, where $t_{i}$ represents firm $i$ 's type, the market demand function is given by $D(t, \cdot)$. Thus, $D(t, p)$ represents aggregate demand in state $t$ when the good is priced at $p$. Each firm $i$ faces a cost function $c_{i}\left(t, q_{i}\right)$ defined on type profiles $t$ and individual output levels $q_{i}$ chosen from a compact subset $Y_{i}$ of $\mathbb{R}_{+}$. Each $Y_{i}$ is endowed with the relativization of the usual Euclidean metric on $\mathbb{R}_{+}$. The common prior over type profiles in $T$ is denoted by $\eta$ (a probability measure over $\left(T, \otimes_{i} \mathscr{T}_{i}\right)$ ), with corresponding marginal probability measures $\eta_{1}$ and $\eta_{2}$.

Each firm $i$ chooses a price $p_{i}$ from a closed and bounded interval $X_{i}$ of $\mathbb{R}_{+}$and a level of supply $q_{i} \in Y_{i}$. Each $X_{i}$ is endowed with the relative Euclidean metric, and the Cartesian products $X_{i} \times Y_{i}$ and $X_{1} \times Y_{1} \times X_{2} \times Y_{2}$ are equipped with the corresponding supremum metric. Given a type profile $t \in T$ and an action profile $\left(p_{1}, q_{1}, p_{2}, q_{2}\right) \in X_{1} \times Y_{1} \times X_{2} \times Y_{2}$, the demand facing firm $i$ is given by

$$
D_{i}\left(t, p_{1}, q_{1}, p_{2}, q_{2}\right):= \begin{cases}D\left(t, p_{i}\right) & \text { if } p_{i}<p_{-i}, \\ G_{i}\left(t, p, q_{1}, q_{2}\right) & \text { if } p_{1}=p_{2}=p \\ H_{i}\left(t, p_{1}, p_{2}, q_{-i}\right) & \text { if } p_{i}>p_{-i} .\end{cases}
$$

We make the following assumptions:

Assumption I. $\eta$ is absolutely continuous with respect to $\eta_{1} \otimes \eta_{2}$.

Assumption J. $\min X_{1}=\min X_{2}=: 0$.

Assumption K. For each $i, c_{i}: T \times Y_{i} \rightarrow \mathbb{R}_{+}$is a bounded and $\left(\left[\otimes_{i=1}^{N} \mathscr{T}_{i}\right] \otimes \mathscr{B}\left(Y_{i}\right), \mathscr{B}(\mathbb{R})\right)$ measurable map such that for each $t \in T$, the map $c_{1}(t, \cdot)+c_{2}(t, \cdot)$ is lower semicontinuous on $Y_{1} \times Y_{2}$.

Assumption L. The map $D: T \times \mathbb{R}_{+} \rightarrow \mathbb{R}_{+}$is a bounded and $\left(\left[\otimes_{i=1}^{N} \mathscr{T}_{i}\right] \otimes \mathscr{B}\left(\mathbb{R}_{+}\right), \mathscr{B}(\mathbb{R})\right)$-measurable map such that the family of maps $\{D(t, \cdot): t \in T\}$ is equicontinuous on $\mathbb{R}_{+}$.

Assumption M. For each $i, G_{i}: T \times X_{i} \times Y_{1} \times Y_{2} \rightarrow \mathbb{R}_{+}$is $\left(\left[\otimes_{i=1}^{N} \mathscr{T}_{i}\right] \otimes \mathscr{B}\left(X_{i}\right) \otimes \mathscr{B}\left(Y_{1}\right) \otimes \mathscr{B}\left(Y_{2}\right), \mathscr{B}(\mathbb{R})\right)$ measurable and bounded and satisfies the following conditions: $G_{i}>0$ if $q_{i}>0 ; G_{i} \geq G_{-i}$ if $q_{i} \geq q_{-i} ;$ and $G_{1}\left(t, p, q_{1}, q_{2}\right)+G_{2}\left(t, p, q_{1}, q_{2}\right)=D(t, p)$.

Assumption N. For each $i, H_{i}: T \times X_{1} \times X_{2} \times Y_{-i} \rightarrow \mathbb{R}_{+}$is $\left(\left[\otimes_{i=1}^{N} \mathscr{T}_{i}\right] \otimes \mathscr{B}\left(X_{1}\right) \otimes \mathscr{B}\left(X_{2}\right) \otimes \mathscr{B}\left(Y_{-i}\right), \mathscr{B}(\mathbb{R})\right)$ measurable and bounded and satisfies the following conditions: $\left\{H_{i}(t, \cdot): t \in T\right\}$ is equicontinuous on $X_{1} \times X_{2} \times Y_{-i} ; H_{i}\left(t, p_{1}, p_{2}, q_{-i}\right) \leq D\left(t, p_{i}\right)$ for each $\left(t, p_{1}, p_{2}, q_{-i}\right)$; and $H_{i}\left(t, p, p, q_{-i}\right)=$ $\max \left\{D(t, p)-q_{-i}, 0\right\}$ for each $\left(t, p, q_{-i}\right)$.

Assumption O. For each $i$ and $\left(t, p, q_{1}, q_{2}\right) \in T \times\left(X_{1} \cap X_{2}\right) \times Y_{1} \times Y_{2}$,

$\min \left\{q_{i}, D(t, p)\right\}+\min \left\{q_{-i}, \max \left\{D(t, p)-q_{i}, 0\right\}\right\} \leq \min \left\{q_{i}, G_{i}\left(t, p, q_{1}, q_{2}\right)\right\}+\min \left\{q_{-i}, G_{-i}\left(t, p, q_{1}, q_{2}\right)\right\}$.

Remark 8. For each $i$ and $\left(t, p, q_{1}, q_{2}\right) \in T \times\left(X_{1} \cap X_{2}\right) \times Y_{1} \times Y_{2}$ we have $\min \left\{q_{i}, D(t, p)\right\} \geq$ $\min \left\{q_{i}, G_{i}\left(t, p, q_{1}, q_{2}\right)\right\}$. Therefore, Assumption O implies

$$
\min \left\{q_{-i}, G_{-i}\left(t, p, q_{1}, q_{2}\right)\right\} \geq \min \left\{q_{-i}, \max \left\{D(t, p)-q_{i}, 0\right\}\right\} .
$$


As an example, one may define, for a continuous and strictly increasing $\operatorname{map} \zeta:[0,1] \rightarrow$ $[0,1]$ with $\zeta(0)=0, \zeta\left(\frac{1}{2}\right)=\frac{1}{2}, \zeta(1)=1$, and, for each $\left(t, p, q_{1}, q_{2}\right)$ with $q_{1}+q_{2}=D(t, p)$,

$$
\begin{aligned}
& D(t, p)-q_{2}=\zeta\left(\frac{q_{1}}{q_{1}+q_{2}}\right) D(t, p) \\
& \begin{cases}\zeta\left(\frac{q_{1}}{q_{1}+q_{2}}\right) D(t, p) & \text { if } D(t, p) \geq q_{1}+q_{2}>0, \zeta\left(\frac{q_{1}}{q_{1}+q_{2}}\right) D(t, p) \geq q_{1} \\
& \text { and }\left(1-\zeta\left(\frac{q_{1}}{q_{1}+q_{2}}\right)\right) D(t, p) \geq q_{2}, \\
q_{1} & \text { if } D(t, p) \geq q_{1}+q_{2}>0, \zeta\left(\frac{q_{1}}{q_{1}+q_{2}}\right) D(t, p)<q_{1}\end{cases} \\
& G_{1}\left(t, p, q_{1}, q_{2}\right):= \begin{cases} & \text { and }\left(1-\zeta\left(\frac{q_{1}}{q_{1}+q_{2}}\right)\right) D(t, p) \geq q_{2}, \\
D(t, p)-q_{2} & \text { if } D(t, p) \geq q_{1}+q_{2}>0, \zeta\left(\frac{q_{1}}{q_{1}+q_{2}}\right) D(t, p) \geq q_{1}, \\
& \text { and }\left(1-\zeta\left(\frac{q_{1}}{q_{1}+q_{2}}\right)\right) D(t, p)<q_{2}, \\
\zeta\left(\frac{q_{1}}{q_{1}+q_{2}}\right) D(t, p) & \text { if } q_{1}+q_{2}>0, \zeta\left(\frac{q_{1}}{q_{1}+q_{2}}\right) D(t, p) \leq q_{1},\end{cases} \\
& \zeta\left(\frac{q_{1}}{q_{1}+q_{2}}\right) D(t, p) \quad \text { if } q_{1}+q_{2}>0, \zeta\left(\frac{q_{1}}{q_{1}+q_{2}}\right) D(t, p) \leq q_{1}, \\
& \text { and }\left(1-\zeta\left(\frac{q_{1}}{q_{1}+q_{2}}\right)\right) D(t, p) \leq q_{2} \text {, } \\
& D(t, p)-q_{2} \quad \text { if } D(t, p)<q_{1}+q_{2}>0 \text { and }\left(1-\zeta\left(\frac{q_{1}}{q_{1}+q_{2}}\right)\right) D(t, p)>q_{2} \text {, }
\end{aligned}
$$

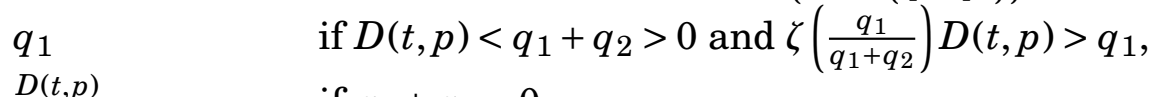

$$
\begin{aligned}
& \text { if } q_{1}+q_{2}=0 \text {, } \\
& \begin{cases}\left(1-\zeta\left(\frac{q_{1}}{q_{1}+q_{2}}\right)\right) D(t, p) & \text { if } D(t, p) \geq q_{1}+q_{2}>0, \zeta\left(\frac{q_{1}}{q_{1}+q_{2}}\right) D(t, p) \geq q_{1}, \\
& \text { and }\left(1-\zeta\left(\frac{q_{1}}{q_{1}+q_{2}}\right)\right) D(t, p) \geq q_{2}, \\
D(t, p)-q_{1} & \text { if } D(t, p) \geq q_{1}+q_{2}>0, \zeta\left(\frac{q_{1}}{q_{1}+q_{2}}\right) D(t, p)<q_{1},\end{cases} \\
& \text { and }\left(1-\zeta\left(\frac{q_{1}}{q_{1}+q_{2}}\right)\right) D(t, p) \geq q_{2} \text {, } \\
& \text { if } D(t, p) \geq q_{1}+q_{2}>0, \zeta\left(\frac{q_{1}}{q_{1}+q_{2}}\right) D(t, p) \geq q_{1} \text {, } \\
& \text { and }\left(1-\zeta\left(\frac{q_{1}}{q_{1}+q_{2}}\right)\right) D(t, p)<q_{2} \text {, } \\
& \left(1-\zeta\left(\frac{q_{1}}{q_{1}+q_{2}}\right)\right) D(t, p) \quad \text { if } q_{1}+q_{2}>0, \zeta\left(\frac{q_{1}}{q_{1}+q_{2}}\right) D(t, p) \leq q_{1} \text {, } \\
& \text { and }\left(1-\zeta\left(\frac{q_{1}}{q_{1}+q_{2}}\right)\right) D(t, p) \leq q_{2} \text {, } \\
& q_{2} \\
& D(t, p)-q_{1} \quad \text { if } D(t, p)<q_{1}+q_{2}>0 \text { and } \zeta\left(\frac{q_{1}}{q_{1}+q_{2}}\right) D(t, p)>q_{1} \text {, } \\
& \frac{D(t, p)}{2} \quad \text { if } q_{1}+q_{2}=0 \text {, }
\end{aligned}
$$

and, for each $i$,

$$
H_{i}\left(t, p_{1}, p_{2}, q_{-i}\right):=\max \left\{0, \frac{D\left(t, p_{i}\right)\left(D\left(t, p_{-i}\right)-q_{-i}\right)}{D\left(t, p_{-i}\right)}\right\} .
$$

These functions are consistent with Assumptions M-O. This example includes, as a particular case, the model used in Section 2.2 of Dasgupta and Maskin [24] (set $\zeta$ equal to the identity function).

The model can be formally described as a Bayesian game

$$
\Gamma:=\left(\left(T_{i}, \mathscr{T}_{i}\right), X_{i} \times Y_{i}, u_{i}, \eta\right)_{i=1}^{2},
$$

where, for each $i$,

$$
u_{i}\left(t, p_{1}, q_{1}, p_{2}, q_{2}\right):=p_{i} \min \left\{q_{i}, D_{i}\left(t, p_{1}, q_{1}, p_{2}, q_{2}\right)\right\}-c_{i}\left(t, q_{i}\right) .
$$


Remark 9. According to (7), the firm that quotes the lower price attracts the entire market demand. When the two firms quote the same price, they split the market in a way that depends on the chosen supplies. In this case Assumption M stipulates that each firm's market share is a monotonic function of its supply and that the firm's market share is positive whenever its supply is positive. As per (7) and Assumption N, the firm that quotes the higher price gets less than full demand, and if the higher price is only slightly above the price quoted by the competitor, the firm's market share is approximately the residual demand as determined by the competitor's capacity (i.e., full market demand minus the competitor's supply). Assumption N subsumes Levitan and Shubik's [44] parallel rationing rule and Edgeworth's [27] proportional rationing rule. Assumption $\mathrm{O}$ says that whenever the firms quote the same price $p$ and choose capacities $q_{1}$ and $q_{2}$, if $G_{1}\left(t, p, q_{1}, q_{2}\right)$ does not constrain the capacity for firm $1, q_{1}$, i.e., if $q_{1} \leq G_{1}\left(t, p, q_{1}, q_{2}\right)$, so that 1 can sell $q_{1}$, then $G_{2}\left(t, p, q_{1}, q_{2}\right)$ does not constrain the capacity for firm 2 below the residual demand $D(t, p)-q_{1}$. This assumption ensures that payoff discontinuities entail a shift in demand from one firm to the other. ${ }^{16,17,18}$

Remark 10. Without Assumption $O$ the sum of the players' payoffs need not be upper semicontinuous. To illustrate this fact, define

$$
G_{i}\left(t, p, q_{1}, q_{2}\right):=\frac{D(t, p)}{2}>0
$$

and

$$
H_{i}\left(t, p_{1}, p_{2}, q_{-i}\right):=\max \left\{0, \frac{D\left(t, p_{i}\right)\left(D\left(t, p_{-i}\right)-q_{-i}\right)}{D\left(t, p_{-i}\right)}\right\},
$$

and suppose that $c_{i} \equiv 0$ for each $i$. Choose $\left(t, p, q_{1}, q_{2}\right) \in T \times\left(X_{1} \cap X_{2}\right) \times Y_{1} \times Y_{2}$ with $p>0$ for which $q_{1}<\frac{D(t, p)}{2}, q_{2}>\frac{D(t, p)}{2}$, and $q_{1}+q_{2}>D(t, p)$. Then

$\min \left\{q_{1}, D(t, p)\right\}+\min \left\{q_{2}, \max \left\{D(t, p)-q_{1}, 0\right\}\right\}=D(t, p)$

$$
\begin{aligned}
& >q_{1}+\frac{D(t, p)}{2} \\
& =\min \left\{q_{1}, G_{1}\left(t, p, q_{1}, q_{2}\right)\right\}+\min \left\{q_{2}, G_{2}\left(t, p, q_{1}, q_{2}\right)\right\},
\end{aligned}
$$

implying that Assumption $\mathrm{O}$ is violated. If $\left(p_{1}^{n}, p_{2}^{n}\right)$ is a sequence converging to $(p, p)$ with $p_{1}^{n}<p_{2}^{n}$ for each $n$, then by continuity of $D(t, \cdot)$ we have $D\left(t, p_{1}^{n}\right) \rightarrow D(t, p)$ and

$$
H_{2}\left(t, p_{1}^{n}, p_{2}^{n}, q_{1}\right) \rightarrow \max \left\{0, \frac{D(t, p)\left(D(t, p)-q_{1}\right)}{D(t, p)}\right\}=\max \left\{0, D(t, p)-q_{1}\right\}=D(t, p)-q_{1},
$$

so that

$u_{1}\left(t, p_{1}^{n}, q_{1}, p_{2}^{n}, q_{2}\right)+u_{2}\left(t, p_{1}^{n}, q_{1}, p_{2}^{n}, q_{2}\right)=p_{1}^{n} \min \left\{q_{1}, D\left(t, p_{1}^{n}\right)\right\}+p_{2}^{n} \min \left\{q_{2}, H_{2}\left(t, p_{1}^{n}, p_{2}^{n}, q_{1}\right)\right\} \rightarrow p D(t, p)$.

However,

$$
\begin{aligned}
u_{1}\left(t, p, q_{1}, p, q_{2}\right)+u_{2}\left(t, p, q_{1}, p, q_{2}\right) & =p \min \left\{q_{1}, G_{1}\left(t, p, q_{1}, q_{2}\right)\right\}+p \min \left\{q_{2}, G_{2}\left(t, p, q_{1}, q_{2}\right)\right\} \\
& =p\left(q_{1}+\frac{D(t, p)}{2}\right)
\end{aligned}
$$

implying that the sum of the players' payoffs is not upper semicontinuous at $\left(p, p, q_{1}, q_{2}\right)$.

\footnotetext{
${ }^{16}$ As per Remark 8, Assumption O implies that a firm's profit when both firms quote the same price cannot be less than the profit the firm would obtain if it were undercut by the competitor.

${ }^{17}$ Assumption O cannot be dispensed with. See Remark 10.

${ }^{18}$ As per the discussion in Remark 5, here a weakening of Assumptions $\mathrm{L}$ and $\mathrm{N}$ is likely to suffice for the conclusion of Corollary 5 .
} 
Corollary 5 (to Theorem 1). Under Assumptions I-O, the game $\Gamma$ defined in (8) possesses a Bayes-Nash equilibrium.

The proof of Corollary 5 is provided in Subsection A.2.3 of the Appendix.

\subsection{Equilibrium existence in imperfectly discriminating contests}

Contests and rent seeking games in the presence of complete information have numerous applications in economics and political science ( $c f$. Tullock [74]). In a perfectly discriminating contest, the prize is awarded to a player who exerts the greatest effort (or expends the largest amount of resources or makes the largest political contribution). In an imperfectly discriminating contest, the agent who expends the greatest effort has the highest probability of winning but this probability may be less than one. For examples and analyses of imperfectly discriminating contests with complete information, see, e.g., Blavatskyy [15], Szymanski [73], Nitzan [54, 55], Nti [57], and Rosen [65]. For a model of an imperfectly discriminating contest with incomplete information and continuous payoffs, see Wasser [78], which proves the existence of a monotone pure strategy equilibrium using the results of Athey [5].

If there is a positive probability that the prize is not a awarded to any player, the sum of payoffs in the game theoretic formulation need not be upper semicontinuous. This is exactly the situation in a rent seeking game in Prokopovych and Yannelis [58] formulated as an imperfectly discriminating contest. In this model, two players compete for a political favor but the favor may be witheld by the grantor unless both players make positive contributions.

We will consider an incomplete information generalization of this example with interdependent valuations in which the common value of the prize depends on the players' private information: if $t=\left(t_{1}, t_{2}\right)$ is the information of player $i$, then the prize has value $v(t)$. More formally, let $v: T_{1} \times T_{2} \rightarrow \mathbb{R}_{+}$be a bounded, measurable function such that, for some $\bar{v}>0$, $\bar{v} \leq v\left(t_{1}, t_{2}\right)$ for all $\left(t_{1}, t_{2}\right) \in T$.

To formally describe the contest success function, let $\pi_{i}:\left(0, \alpha_{1}\right] \times\left(0, \alpha_{2}\right] \rightarrow \mathbb{R}_{++}, i \in\{1,2\}$, be continuous functions satisfying the following:

(i) $\pi_{1}\left(x_{1}, x_{2}\right)+\pi_{2}\left(x_{1}, x_{2}\right)=1$.

(ii) For each $i, x_{-i} \mapsto \pi_{i}\left(x_{i}, x_{-i}\right)$ is strictly decreasing for each $x_{i}$ and $x_{i} \mapsto \pi_{i}\left(x_{i}, x_{-i}\right)$ is strictly increasing for each $x_{-i}$.

(iii) For each $i, \pi_{i}\left(\alpha_{1}, \alpha_{2}\right)<1$.

(iv) For each $x_{i} \in\left(0, \alpha_{i}\right], \lim _{y_{-i} \rightarrow 0+} \pi_{i}\left(x_{i}, y_{-i}\right)=1$.

To complete the definition of each $\pi_{i}$, suppose that $0<\lambda \leq \mu<1$ and $1+\lambda-2 \mu \geq 0$, and define

$$
\begin{aligned}
\pi_{i}\left(x_{i}, 0\right)=\mu & \text { if } x_{i}>0, \\
\pi_{i}\left(0, x_{-i}\right)=0 & \text { if } x_{-i}>0
\end{aligned}
$$

and

$$
\pi_{1}(0,0)=\lambda=\pi_{2}(0,0) .
$$

The payoff to player $i$ is defined as

$$
u_{i}\left(t, x_{1}, x_{2}\right):=\pi_{i}\left(x_{1}, x_{2}\right) v(t)-x_{i} .
$$

The associated Bayesian game is

$$
\Gamma:=\left(\left(T_{i}, \mathscr{T}_{i}\right), X_{i}, u_{i}, p\right)_{i=1}^{2},
$$


where $X_{i}:=\left[0, \alpha_{i}\right]$ and $u_{i}$ is given by (9) for each $i$ and where $p$ is assumed absolutely continuous with respect to the product of its marginals $p_{1} \otimes p_{2}$.

Note that the sum of payoffs is given by

$$
u_{1}\left(t, x_{1}, x_{2}\right)+u_{1}\left(t, x_{1}, x_{2}\right)= \begin{cases}v(t)-x_{1}-x_{2} & \text { if } x_{i} \in\left(0, \alpha_{i}\right] \text { for each } i, \\ \mu v(t)-x_{i} & \text { if } x_{i} \in\left(0, \alpha_{i}\right] \text { and } x_{-i}=0, \\ 2 \lambda v(t) & \text { if }\left(x_{1}, x_{2}\right)=(0,0),\end{cases}
$$

and therefore this sum is not upper semicontinuous on $X_{1} \times X_{2}$ for fixed $t$.

Our nonsymmetric incomplete information model includes as a special case the symmetric example with complete information of Prokopovych and Yannelis [58] in which each $\alpha_{i}=2$,

$$
\pi_{i}\left(x_{i}, x_{-i}\right)=\frac{x_{i}^{3}}{x_{1}^{3}+x_{2}^{3}} \quad \text { if } x_{i} \in(0,2] \text { for each } i,
$$

$v(\cdot) \equiv 2, \mu=\frac{1}{2}$, and $\lambda=\frac{1}{4}$. They show that, for these parameters, the game does not have a pure strategy equilibrium. However, the game does have a mixed strategy equilibrium; indeed, as shown in [58], it satisfies uniform diagonal security. Thanks to Theorem 2 we can extend their observations to the incomplete information framework.

Corollary 6 (to Theorem 2). The game $\Gamma$ defined in (10) possesses a Bayes-Nash equilibrium.

The proof of Corollary 6 is relegated to Subsection A.2.4 of the Appendix.

\section{A Appendix}

\section{A.1 Proofs of Lemma 2, Lemma 3, and Lemma 4}

\section{A.1.1 Preliminary lemmas}

Lemma 5. Suppose that the Bayesian game $\left(\left(T_{i}, \mathscr{T}_{i}\right), X_{i}, u_{i}, p\right)_{i=1}^{N}$ is uniformly payoff secure. If $p$ is absolutely continuous with respect to $p_{1} \otimes \cdots \otimes p_{N}$, then for each $i, \varepsilon>0$, and $s_{i} \in \mathscr{P}_{i}$, there exists $s_{i}^{*} \in \mathscr{P}_{i}$ such that for every $\sigma_{-i} \in \mathscr{Y}_{-i}$, there exists a neighborhood $V_{\sigma_{-i}}$ of $\sigma_{-i}$ such that

$$
U_{i}\left(s_{i}^{*}, v_{-i}\right)>U_{i}\left(s_{i}, \sigma_{-i}\right)-\varepsilon, \text { for all } v_{-i} \in V_{\sigma_{-i}} .
$$

Proof. Fix $i, \varepsilon>0$, and $s_{i} \in \mathscr{P}_{i}$. Let $f$ be a density of $p$ with respect to $p_{1} \otimes \cdots \otimes p_{N}$. To lighten the notation, let $P:=\otimes_{i=1}^{N} p_{i}$. Let $\mathscr{T}^{*}(P)$ denote the $P$-completion of $\mathscr{T}$ and let $P^{*}$ denote the unique extension of $P$ to $\mathscr{T}^{*}(P)$. Let

$$
\mathscr{T}^{*}:=\bigcap_{P \in \Delta(T, \mathscr{T})} \mathscr{T}^{*}(P)
$$

denote the universal completion of $\mathscr{T}$. Note that $\mathscr{T} \subseteq \mathscr{T}^{*} \subseteq \mathscr{T}^{*}(P)$ and, abusing notation slightly, we will use $P^{*}$ for the restriction of $P^{*}$ to $\mathscr{T}^{*}$. Note that if $h: T \rightarrow \mathbb{R}$ is a bounded, $(\mathscr{T}, \mathscr{B}(\mathbb{R}))$-measurable map, then $h$ is a bounded $\left(\mathscr{T}^{*}, \mathscr{B}(\mathbb{R})\right)$-measurable map and

$$
\int_{T} h(t) P(d t)=\int_{T} h(t) P^{*}(d t) .
$$

The proof proceeds in four steps.

Step 1. Uniform payoff security gives $s_{i}^{*} \in \mathscr{P}_{i}$ such that for every $\left(t, x_{-i}\right) \in T \times X_{-i}$, there is a neighborhood $V_{x_{-i}}$ of $x_{-i}$ such that

$$
u_{i}\left(t,\left(s_{i}^{*}\left(t_{i}\right), y_{-i}\right)\right)>u_{i}\left(t,\left(s_{i}\left(t_{i}\right), x_{-i}\right)\right)-\frac{\varepsilon}{2}, \quad \text { for all } y_{-i} \in V_{x_{-i}} .
$$


Therefore, for every $\left(t, x_{-i}\right) \in T \times X_{-i}$ there is a neighborhood $V_{x_{-i}}$ of $x_{-i}$ such that

$$
u_{i}\left(t,\left(s_{i}^{*}\left(t_{i}\right), y_{-i}\right)\right) f(t) \geq\left(u_{i}\left(t,\left(s_{i}\left(t_{i}\right), x_{-i}\right)\right)-\frac{\varepsilon}{2}\right) f(t), \quad \text { for all } y_{-i} \in V_{x_{-i}} .
$$

Define $\xi: T \times X \rightarrow \mathbb{R}$ by

$$
\xi(t, x):=\sup _{n \in \mathbb{N}} \inf _{y \in N_{\frac{1}{n}}(x)} u_{i}\left(t,\left(s_{i}^{*}\left(t_{i}\right), y_{-i}\right)\right) f(t) .
$$

By the Theorem in Carbonell-Nicolau [18], $\xi$ is a $\left(\mathscr{T}^{*} \otimes \mathscr{B}(X), \mathscr{B}(\mathbb{R})\right)$-measurable map.

Step 2. Let $\mathscr{R}$ (resp. $\mathscr{R}^{*}$ ) denote the set of transition probabilities with respect to $(T, \mathscr{T}$ ) (resp. $\left(T, \mathscr{T}^{*}\right)$ ) and $(X, \mathscr{B}(X))$. Then $\mathscr{R} \subseteq \mathscr{R}^{*}$ since $\mathscr{T} \subseteq \mathscr{T}^{*}$. If $\mathscr{R}$ is endowed with the relative topology inherited from the $P^{*}$-narrow topology on $\mathscr{R}^{*}$, then the inclusion map is continuous. We will show that the inclusion map is continuous when $\mathscr{R}$ is endowed with the $P$-narrow topology. This fact will be used in Step 3 below.

Suppose that $\left(\rho^{\alpha}\right)$ is a net in $\mathscr{R}$ and suppose that $\rho^{\alpha} \rightarrow \rho$ in the $P$-narrow topology on $\mathscr{R}$. We must show that $\rho^{\alpha} \rightarrow \rho$ in $\mathscr{R}^{*}$ when $\mathscr{R}^{*}$ is endowed with the $P^{*}$-narrow topology.

Suppose that $A \in \mathscr{T}^{*}$ and $g: X \rightarrow \mathbb{R}$ is bounded and continuous. Applying Theorem 2.2 in Balder [6], we need to show that

$$
\int_{T} \int_{X} \chi_{A}(t) g(x) \rho^{\alpha}(d x \mid t) P^{*}(d t) \rightarrow \int_{T} \int_{X} \chi_{A}(t) g(x) \rho(d x \mid t) P^{*}(d t) .
$$

Since $A \in \mathscr{T}^{*}$ and $\mathscr{T}^{*} \subseteq \mathscr{T}^{*}(P)$, there exists $B \in \mathscr{T}$ such that $B \subseteq A, P(B)=P^{*}(A)$, and $P^{*}(A \backslash B)=0$. Observing that

$$
t \mapsto \chi_{B}(t) \int_{X} g(x) \rho^{\alpha}(d x \mid t) \quad \text { and } \quad t \mapsto \chi_{B}(t) \int_{X} g(x) \rho(d x \mid t)
$$

are bounded, $(\mathscr{T}, \mathscr{B}(\mathbb{R}))$-measurable maps, we conclude that

$$
\begin{aligned}
\int_{T} \chi_{A}(t)\left[\int_{X} g(x) \rho^{\alpha}(d x \mid t)\right] P^{*}(d t) & =\int_{T} \chi_{B}(t)\left[\int_{X} g(x) \rho^{\alpha}(d x \mid t)\right] P^{*}(d t) \\
& =\int_{T} \chi_{B}(t)\left[\int_{X} g(x) \rho^{\alpha}(d x \mid t)\right] P(d t)
\end{aligned}
$$

and

$$
\begin{aligned}
\int_{T} \chi_{A}(t)\left[\int_{X} g(x) \rho(d x \mid t)\right] P^{*}(d t) & =\int_{T} \chi_{B}(t)\left[\int_{X} g(x) \rho(d x \mid t)\right] P^{*}(d t) \\
& =\int_{T} \chi_{B}(t)\left[\int_{X} g(x) \rho(d x \mid t)\right] P(d t) .
\end{aligned}
$$

Recalling that $\rho^{\alpha} \rightarrow \rho$ in the $P$-narrow topology on $\mathscr{R}$, it follows that

$$
\int_{T} \chi_{B}(t)\left[\int_{X} g(x) \rho^{\alpha}(d x \mid t)\right] P(d t) \rightarrow \int_{T} \chi_{B}(t)\left[\int_{X} g(x) \rho(d x \mid t)\right] P(d t),
$$

and we obtain the desired conclusion.

Step 3. Now choose $\sigma=\left(\sigma_{1}, . ., \sigma_{N}\right) \in \mathscr{Y}$, define $Q(\sigma) \in \mathscr{R}$ as

$$
Q(\sigma)(\cdot \mid t):=\sigma_{1}\left(\cdot \mid t_{1}\right) \otimes \cdots \otimes \sigma_{N}\left(\cdot \mid t_{N}\right), \quad \text { for every } t \in T,
$$

and note that $Q(\sigma) \in \mathscr{R}^{*}$. Since $\xi$ is $\left(\mathscr{T}^{*} \otimes \mathscr{B}(X), \mathscr{B}(\mathbb{R})\right)$-measurable (Step 1$)$ and since the map $x \mapsto \xi(t, x)$ defined on $X$ is lower semicontinuous for each $t \in T$, we can apply Theorem 
2.2(a) in Balder [6] and deduce the existence of a $P^{*}$-narrow open set $W$ in $\mathscr{R}^{*}$ containing $Q(\sigma)$ such that

$$
\int_{T} \int_{X} \xi(t, x) \tau(d x \mid t) P^{*}(d t)>\int_{T} \int_{X} \xi(t, x)\left[\sigma_{1}\left(d x_{1} \mid t_{1}\right) \otimes \cdots \otimes \sigma\left(d x_{N} \mid t_{N}\right)\right] P^{*}(d t)-\frac{\varepsilon}{2}
$$

for all $\tau \in W$. Applying the result of Step 2, there exists a $P$-narrow open set $V$ in $\mathscr{R}$ such $Q(\sigma) \in V$ and

$$
\int_{T} \int_{X} \xi(t, x) v(d x \mid t) P^{*}(d t)>\int_{T} \int_{X} \xi(t, x)\left[\sigma_{1}\left(d x_{1} \mid t_{1}\right) \otimes \cdots \otimes \sigma\left(d x_{N} \mid t_{N}\right)\right] P^{*}(d t)-\frac{\varepsilon}{2}
$$

for all $v \in V$.

Applying Theorem 2.5 in Balder [6], it follows that the map $v \in \mathscr{Y} \mapsto Q(v) \in \mathscr{R}$ is continuous when $\mathscr{Y}$ is endowed with the product topology generated by the $p_{i}$-narrow topology on each factor $\mathscr{Y}_{i}$. Therefore, there exists an open set $V_{\sigma}$ (i.e., open with respect to this product topology) containing $\sigma$ such that

$$
\begin{aligned}
\int_{T} \int_{X} \xi(t, x)\left[v_{1}\left(d x_{1} \mid t_{1}\right) \otimes \cdots \otimes v_{N}\left(d x_{N} \mid t_{N}\right)\right] P^{*}(d t) & \\
& >\int_{T} \int_{X} \xi(t, x)\left[\sigma_{1}\left(d x_{1} \mid t_{1}\right) \otimes \cdots \otimes \sigma_{N}\left(d x_{N} \mid t_{N}\right)\right] P^{*}(d t)-\frac{\varepsilon}{2}
\end{aligned}
$$

for all $\left(v_{1}, . ., v_{N}\right) \in V_{\sigma}$.

Step 4. Recall that for each $\left(t, x_{-i}\right) \in T \times X_{-i}$, there is a neighborhood $V_{x_{-i}}$ of $x_{-i}$ such that (12) holds. Consequently, $(t, x) \in T \times X$ implies that

$$
u_{i}\left(t,\left(s_{i}^{*}\left(t_{i}\right), x_{-i}\right)\right) f(t) \geq \xi(t, x) \geq\left(u_{i}\left(t,\left(s_{i}\left(t_{i}\right), x_{-i}\right)\right)-\frac{\varepsilon}{2}\right) f(t) .
$$

This, together with the conclusion in Step 3, implies that for every $\left(v_{1}, . ., v_{N}\right) \in V_{\sigma}$,

$$
\begin{aligned}
U_{i}\left(s_{i}^{*}, v_{-i}\right) & =\int_{T} \int_{X}\left[u_{i}\left(t,\left(s_{i}^{*}\left(t_{i}\right), x_{-i}\right)\right) f(t)\right]\left[v_{1}\left(d x_{1} \mid t_{1}\right) \otimes \cdots \otimes v_{N}\left(d x_{N} \mid t_{N}\right)\right] P(d t) \\
& =\int_{T} \int_{X}\left[u_{i}\left(t,\left(s_{i}^{*}\left(t_{i}\right), x_{-i}\right)\right) f(t)\right]\left[v_{1}\left(d x_{1} \mid t_{1}\right) \otimes \cdots \otimes v_{N}\left(d x_{N} \mid t_{N}\right)\right] P^{*}(d t) \\
& \geq \int_{T} \int_{X} \xi(t, x)\left[v_{1}\left(d x_{1} \mid t_{1}\right) \otimes \cdots \otimes v_{N}\left(d x_{N} \mid t_{N}\right)\right] P^{*}(d t) \\
& >\int_{T} \int_{X} \xi(t, x)\left[\sigma_{1}\left(d x_{1} \mid t_{1}\right) \otimes \cdots \otimes \sigma_{N}\left(d x_{N} \mid t_{N}\right)\right] P^{*}(d t)-\frac{\varepsilon}{2} \\
& \geq \int_{T} \int_{X}\left[u_{i}\left(t,\left(s_{i}\left(t_{i}\right), x_{-i}\right)\right) f(t)\right]\left[\sigma_{1}\left(d x_{1} \mid t_{1}\right) \otimes \cdots \otimes \sigma_{N}\left(d x_{N} \mid t_{N}\right)\right] P^{*}(d t)-\varepsilon \\
& =\int_{T} \int_{X}\left[u_{i}\left(t,\left(s_{i}\left(t_{i}\right), x_{-i}\right)\right) f(t)\right]\left[\sigma_{1}\left(d x_{1} \mid t_{1}\right) \otimes \cdots \otimes \sigma_{N}\left(d x_{N} \mid t_{N}\right)\right] P(d t)-\varepsilon \\
& =U_{i}\left(s_{i}, \sigma_{-i}\right)-\varepsilon .
\end{aligned}
$$

This establishes (11).

Lemma 6. Suppose that the Bayesian game $\left(\left(T_{i}, \mathscr{T}_{i}\right), X_{i}, u_{i}, p\right)_{i=1}^{N}$ is uniformly diagonally secure. If $p$ is absolutely continuous with respect to $p_{1} \otimes \cdots \otimes p_{N}$, then for each $\varepsilon>0$ and $s \in \mathscr{P}$, there exists $s^{*} \in \mathscr{P}$ such that for every $\sigma \in \mathscr{Y}$, there exists a neighborhood $V_{\sigma}$ of $\sigma$ such that

$$
\sum_{i=1}^{N} U_{i}\left(s_{i}^{*}, v_{-i}\right)-\sum_{i=1}^{N} U_{i}(v)>\sum_{i=1}^{N} U_{i}\left(s_{i}, \sigma_{-i}\right)-\sum_{i=1}^{N} U_{i}(\sigma)-\varepsilon, \quad \text { for all } v \in V_{\sigma} .
$$


Proof. Fix $\varepsilon>0$ and $s \in \mathscr{P}$. Let $f$ be a density of $p$ with respect to $p_{1} \otimes \cdots \otimes p_{N}$. Uniform diagonal security gives $s^{*} \in \mathscr{P}$ such that for all $(t, x) \in T \times X$, there exists a neighborhood $V_{x}$ of $x$ such that

$$
\sum_{i=1}^{N} u_{i}\left(t,\left(s_{i}^{*}\left(t_{i}\right), y_{-i}\right)\right)-\sum_{i=1}^{N} u_{i}(t, y)>\sum_{i=1}^{N} u_{i}\left(t,\left(s_{i}\left(t_{i}\right), x_{-i}\right)\right)-\sum_{i=1}^{N} u_{i}(t, x)-\frac{\varepsilon}{2}, \quad \text { for all } y \in V_{x} .
$$

Therefore, for all $(t, x) \in T \times X$ there exists a neighborhood $V_{x}$ of $x$ such that

$$
\left(\sum_{i=1}^{N} u_{i}\left(t,\left(s_{i}^{*}\left(t_{i}\right), y_{-i}\right)\right)-\sum_{i=1}^{N} u_{i}(t, y)\right) f(t) \geq\left(\sum_{i=1}^{N} u_{i}\left(t,\left(s_{i}\left(t_{i}\right), x_{-i}\right)\right)-\sum_{i=1}^{N} u_{i}(t, x)-\frac{\varepsilon}{2}\right) f(t)
$$

for all $y \in V_{x}$.

Define $\xi: T \times X \rightarrow \mathbb{R}$ by

$$
\xi(t, x):=\sup _{n \in \mathbb{N}} \inf _{y \in N_{\frac{1}{n}}(x)}\left[\left(\sum_{i=1}^{N} u_{i}\left(t,\left(s_{i}^{*}\left(t_{i}\right), y_{-i}\right)\right)-\sum_{i=1}^{N} u_{i}(t, y)\right) f(t)\right] .
$$

By the Theorem in Carbonell-Nicolau [18], $\xi$ is a $\left(\mathscr{T}^{*} \otimes \mathscr{B}(X), \mathscr{B}(\mathbb{R})\right)$-measurable map where $\mathscr{T}^{*}$ denotes the universal completion of $\mathscr{T}$.

Define two functions $H: T \times X \rightarrow \mathbb{R}$ and $H^{*}: T \times X \rightarrow \mathbb{R}$ by

$$
H^{*}(t, x):=\sum_{i=1}^{N} u_{i}\left(t,\left(s_{i}^{*}\left(t_{i}\right), x_{-i}\right)\right)-\sum_{i=1}^{N} u_{i}(t, x)
$$

and

$$
H(t, x):=\sum_{i=1}^{N} u_{i}\left(t,\left(s_{i}\left(t_{i}\right), x_{-i}\right)\right)-\sum_{i=1}^{N} u_{i}(t, x)
$$

The proof is now completed as a verbatim transcription of the proof of Lemma 5 above with $u_{i}\left(t,\left(s_{i}^{*}\left(t_{i}\right), x_{-i}\right)\right)$ replaced by $H^{*}(t, x)$ and $u_{i}\left(t,\left(s_{i}\left(t_{i}\right), x_{-i}\right)\right)$ replaced by $H(t, x)$ for all $(t, x) \in T \times X$.

\section{A.1.2 Proof of Lemma 2}

Lemma 2. Suppose that the Bayesian game $\left(\left(T_{i}, \mathscr{T}_{i}\right), X_{i}, u_{i}, p\right)_{i=1}^{N}$ is uniformly payoff secure. If $p$ is absolutely continuous with respect to $p_{1} \otimes \cdots \otimes p_{N}$, then the game $G^{b}$ defined in (1) is payoff secure.

Proof. Fix $\sigma=\left(\sigma_{1}, \ldots, \sigma_{N}\right) \in \mathscr{Y}, i$, and $\varepsilon>0$. Let $f$ be a density of $p$ with respect to $P=$ $p_{1} \otimes \cdots \otimes p_{N}$. We must show that there exist $\sigma_{i}^{*} \in \mathscr{Y}_{i}$ and a neighborhood $V_{\sigma_{-i}}$ of $\sigma_{-i}$ such that

$$
U_{i}\left(\sigma_{i}^{*}, v_{-i}\right)>U_{i}(\sigma)-\varepsilon, \text { for every } v_{-i} \in V_{\sigma_{-i}} .
$$

We begin by showing that there exists $s_{i} \in \mathscr{P}_{i}$ such that

$$
U_{i}\left(s_{i}, \sigma_{-i}\right) \geq U_{i}(\sigma)-\frac{\varepsilon}{2} .
$$

Let $\mathscr{T}_{i}^{*}\left(p_{i}\right)$ denote the $p_{i}$-completion of $\mathscr{T}_{i}$ and define $H_{i}: T_{i} \times X_{i} \rightarrow \mathbb{R}$ by

$$
H_{i}\left(t_{i}, x_{i}\right):=\int_{T_{-i}} \int_{X_{-i}}\left[u_{i}(t, x) f(t)\right]\left[\underset{j \neq i}{\otimes} \sigma_{j}\left(d x_{j} \mid t_{j}\right)\right] P_{-i}\left(d t_{-i}\right) .
$$

The map $H_{i}$ is $\left(\mathscr{T}_{i} \otimes \mathscr{B}\left(X_{i}\right), \mathscr{B}(\mathbb{R})\right)$-measurable implying that $H_{i}$ is $\left(\mathscr{T}_{i}^{*}\left(p_{i}\right) \otimes \mathscr{B}\left(X_{i}\right), \mathscr{B}(\mathbb{R})\right)$ measurable. Since $\mathscr{T}_{i}^{*}\left(p_{i}\right)$ coincides with its universal completion, it follows from Theorem 
3.1 and Example 2.3 of Rieder [64] that for each $\delta>0$ there is a $\left(\mathscr{T}_{i}^{*}\left(p_{i}\right), \mathscr{B}\left(X_{i}\right)\right)$-measurable $\delta$-maximizer of $H_{i}$, i.e., for every $\delta>0$ there exists a $\left(\mathscr{T}_{i}^{*}\left(p_{i}\right), \mathscr{B}\left(X_{i}\right)\right)$-measurable $s_{i}^{\delta}: T_{i} \rightarrow X_{i}$ such that for every $t_{i} \in T_{i}$,

$$
H_{i}\left(t_{i}, s_{i}^{\delta}\left(t_{i}\right)\right) \geq \sup _{x_{i} \in X_{i}} H_{i}\left(t_{i}, x_{i}\right)-\delta
$$

Applying Theorem 10.35 in Aliprantis and Border [1] there exists a $\left(\mathscr{T}_{i}, \mathscr{B}\left(X_{i}\right)\right)$-measurable map $s_{i}$ and a set $A \in \mathscr{T}_{i}$ such that $p_{i}(A)=0$ and $s_{i}\left(t_{i}\right)=s_{i}^{\frac{\varepsilon}{2}}\left(t_{i}\right)$ for all $t_{i} \in T_{i} \backslash A$. Consequently, we have

$$
\begin{aligned}
U_{i}\left(s_{i}, \sigma_{-i}\right) & =\int_{T_{i}} H_{i}\left(t_{i}, s_{i}\left(t_{i}\right)\right) p_{i}\left(d t_{i}\right) \\
& \geq \int_{T} \int_{X} u_{i}(t, x) f(t) \sigma_{1}\left(d x_{1} \mid t_{1}\right) \cdots \sigma_{N}\left(d x_{N} \mid t_{N}\right) p_{1}\left(d t_{1}\right) \cdots p_{N}\left(d t_{N}\right)-\frac{\varepsilon}{2} \\
& =U_{i}(\sigma)-\frac{\varepsilon}{2} .
\end{aligned}
$$

By Lemma 5, there exist $s_{i}^{*} \in \mathscr{P}_{i}$ and a neighborhood $V_{\sigma_{-i}}$ of $\sigma_{-i}$ such that $U_{i}\left(s_{i}^{*}, v_{-i}\right)>$ $U_{i}\left(s_{i}, \sigma_{-i}\right)-\frac{\varepsilon}{2}$ for all $v_{-i} \in V_{\sigma_{-i}}$. This, together with (14), gives (13).

\section{A.1.3 Proof of Lemma 3}

Lemma 3. Given a Bayesian game $\left(\left(T_{i}, \mathscr{T}_{i}\right), X_{i}, u_{i}, p\right)_{i=1}^{N}$, suppose that for each $t \in T$, the map $\sum_{i=1}^{N} u_{i}(t, \cdot): X \rightarrow \mathbb{R}$ is upper semicontinuous. Suppose further that $p$ is absolutely continuous with respect to $p_{1} \otimes \cdots \otimes p_{N}$. Then the map $\sum_{i=1}^{N} U_{i}(\cdot): \mathscr{Y} \rightarrow \mathbb{R}$ is upper semicontinuous.

Proof. Fix $\sigma \in \mathscr{Y}$ and $\epsilon>0$. Let $f$ be a density of $p$ with respect to $P:=p_{1} \otimes \cdots \otimes p_{N}$. We need to show that there is a neighborhood $V_{\sigma}$ of $\sigma$ such that

$$
\sum_{i=1}^{N} U_{i}(v)<\sum_{i=1}^{N} U_{i}(\sigma)+\epsilon, \quad \text { for all } v \in V_{\sigma} .
$$

Let $F(t, x):=\sum_{i=1}^{N}\left[u_{i}(t, x) f(t)\right]$. As in the proof of Lemma 5 , define $Q(\sigma) \in \mathscr{R}$ as

$$
Q(\sigma)(\cdot \mid t):=\sigma_{1}\left(\cdot \mid t_{1}\right) \otimes \cdots \otimes \sigma_{N}\left(\cdot \mid t_{N}\right), \quad \text { for each } t \in T .
$$

(Recall that $\mathscr{R}$ denotes the set of transition probabilities with respect to $(T, \mathscr{T})$ and $(X, \mathscr{B}(X))$.) Applying Theorem 2.2(a) in Balder [6], it follows that there exists a $P$-narrow open set $W \subseteq \mathscr{R}$ containing $Q(\sigma)$ such that

$$
\int_{T} \int_{X} F(t, x) \tau(d x \mid t) P(d t)<\int_{T} \int_{X} F(t, x)\left[\sigma_{1}\left(d x_{1} \mid t_{1}\right) \otimes \cdots \otimes \sigma\left(d x_{N} \mid t_{N}\right)\right] P(d t)+\epsilon
$$

for all $\tau \in W$. By Theorem 2.5 in Balder [6], the map $v \in \mathscr{Y} \mapsto Q(v) \in \mathscr{R}$ is continuous when $\mathscr{Y}$ is endowed with the product topology generated by the $p_{i}$-narrow topology on each factor $\mathscr{Y}_{i}$. Therefore, there exists an open set $V_{\sigma} \subseteq \mathscr{Y}$ (i.e., open with respect to this product topology) containing $\sigma$ such that

$$
\begin{aligned}
\int_{T} \int_{X} F(t, x)\left[v _ { 1 } ( d x _ { 1 } | t _ { 1 } ) \otimes \cdots \otimes v _ { N } \left(d x_{N} \mid\right.\right. & \left.\left.t_{N}\right)\right] P(d t) \\
& <\int_{T} \int_{X} F(t, x)\left[\sigma_{1}\left(d x_{1} \mid t_{1}\right) \otimes \cdots \otimes \sigma_{N}\left(d x_{N} \mid t_{N}\right)\right] P(d t)+\varepsilon
\end{aligned}
$$

for all $\left(v_{1}, . ., v_{N}\right) \in V_{\sigma}$. This implies (15). 


\section{A.1.4 Proof of Lemma 4}

Lemma 4. Suppose that the Bayesian game $\left(\left(T_{i}, \mathscr{T}_{i}\right), X_{i}, u_{i}, p\right)_{i=1}^{N}$ is uniformly diagonally secure. If $p$ is absolutely continuous with respect to $p_{1} \otimes \cdots \otimes p_{N}$, then the game $G^{b}$ defined in (1) is diagonally transfer continuous.

Proof. Let $f$ be a density of $p$ with respect to $P:=p_{1} \otimes \cdots \otimes p_{N}$. Fix $\sigma=\left(\sigma_{1}, \ldots, \sigma_{N}\right) \in \mathscr{Y}$ and suppose that $\sigma$ is not a Nash equilibrium of $G^{b}$. Then there exists $v \in \mathscr{Y}$ such that

$$
\sum_{i=1}^{N} U_{i}\left(v_{i}, \sigma_{-i}\right)-\sum_{i=1}^{N} U_{i}(\sigma)>0 .
$$

We first show that there exists $s \in \mathscr{P}$ such that

$$
\sum_{i=1}^{N} U_{i}\left(s_{i}, \sigma_{-i}\right)-\sum_{i=1}^{N} U_{i}(\sigma)>0 .
$$

Choose

$$
\varepsilon \in\left(0, \sum_{i=1}^{N} U_{i}\left(v_{i}, \sigma_{-i}\right)-\sum_{i=1}^{N} U_{i}(\sigma)\right)
$$

Fix $i$ and define $H_{i}: T_{i} \times X_{i} \rightarrow \mathbb{R}$ by

$$
H_{i}\left(t_{i}, x_{i}\right):=\int_{T_{-i}} \int_{X_{-i}}\left[u_{i}(t, x) f(t)\right]\left[\underset{j \neq i}{\otimes} \sigma_{j}\left(d x_{j} \mid t_{j}\right)\right] P_{-i}\left(d t_{-i}\right) .
$$

Applying Applying Theorem 10.35 in Aliprantis and Border [1] as in the proof of Lemma 2, there exists a $\left(\mathscr{T}_{i}, \mathscr{B}\left(X_{i}\right)\right)$-measurable map $s_{i}$ and a set $A \in \mathscr{T}_{i}$ such that $p_{i}(A)=0$ and

$$
H_{i}\left(t_{i}, s_{i}\left(t_{i}\right)\right) \geq \sup _{x_{i} \in X_{i}} H_{i}\left(t_{i}, x_{i}\right)-\frac{\varepsilon}{N}, \quad \text { for all } t_{i} \in T_{i} \backslash A .
$$

Consequently,

$$
\begin{aligned}
U_{i}\left(s_{i}, \sigma_{-i}\right) & =\int_{T_{i}} H_{i}\left(t_{i}, s_{i}\left(t_{i}\right)\right) p_{1}\left(d t_{1}\right) \\
& \geq \int_{T_{i}} \int_{X_{i}}\left[\int_{T_{-i}} \int_{X_{-i}}\left[u_{i}(t, x) f(t)\right]\left[\underset{j \neq i}{\otimes} \sigma_{j}\left(d x_{j} \mid t_{j}\right)\right] P_{-i}\left(d t_{-i}\right)\right] v_{i}\left(d x_{i} \mid t_{i}\right) p_{i}\left(d t_{i}\right)-\frac{\varepsilon}{N} \\
& =U_{i}\left(v_{i}, \sigma_{-i}\right)-\frac{\varepsilon}{N}
\end{aligned}
$$

Therefore, a finite number of iterations of the above argument gives $s \in \mathscr{P}$ such that

$$
\sum_{i=1}^{N} U_{i}\left(s_{i}, \sigma_{-i}\right)-\sum_{i=1}^{N} U_{i}(\sigma) \geq \sum_{i=1}^{N} U_{i}\left(v_{i}, \sigma_{-i}\right)-\sum_{i=1}^{N} U_{i}(\sigma)-\varepsilon>0
$$

establishing the inequality (16).

Next, choose

$$
\alpha \in\left(0, \sum_{i=1}^{N} U_{i}\left(s_{i}, \sigma_{-i}\right)-\sum_{i=1}^{N} U_{i}(\sigma)\right) .
$$

By Lemma 6 , there exist $s^{*} \in \mathscr{P}$ and a neighborhood $V_{\sigma}$ of $\sigma$ such that

$$
\sum_{i=1}^{N} U_{i}\left(s_{i}^{*}, v_{-i}\right)-\sum_{i=1}^{N} U_{i}(v)>\sum_{i=1}^{N} U_{i}\left(s_{i}, \sigma_{-i}\right)-\sum_{i=1}^{N} U_{i}(\sigma)-\alpha, \quad \text { for all } v \in V_{\sigma},
$$

Summarizing, there exists a neighborhood $V_{\sigma}$ of $\sigma$ such that

$$
\sum_{i=1}^{N} U_{i}\left(s_{i}^{*}, v_{-i}\right)-\sum_{i=1}^{N} U_{i}(v)>0, \quad \text { for all } v \in V_{\sigma} .
$$

and the proof is complete. 


\section{A.2 Proofs of Corollary 3, Corollary 4, Corollary 5, and Corollary 6}

\section{A.2.1 Proof of Corollary 3}

Corollary 3 (to Theorem 1). Under Assumptions A-D, the auction game $\Gamma$ defined in (4) possesses a Bayes-Nash equilibrium.

Proof. By virtue of Theorem 1, it suffices to show that $\Gamma$ is uniformly payoff secure and the $\operatorname{map} \sum_{i=1}^{N} u_{i}(t, \cdot): X \rightarrow \mathbb{R}$ is upper semicontinuous for each $t \in T$. Because

$$
\sum_{i=1}^{N} u_{i}(t, b)=f(t, b)+(N-1) g(t, b)+\sum_{i=1}^{N} h_{i}(t, b)
$$

the upper semicontinuity of $\sum_{i=1}^{N} u_{i}(t, \cdot)$ follows from Assumption C(i).

To see that $\Gamma$ is uniformly payoff secure, fix $i, \varepsilon>0$, and $s_{i} \in \mathscr{P}_{i}$. By Assumption C(i) and Remark 3, there exists $\delta>0$ such that for all $b \in B$,

$$
\begin{array}{r}
\left|f_{i}(t, b)-f_{i}\left(t, b^{\prime}\right)\right|<\frac{\varepsilon}{4},\left|g_{i}(t, b)-g_{i}\left(t, b^{\prime}\right)\right|<\frac{\varepsilon}{4}, \text { and }\left|h_{i}(t, b)-h_{i}\left(t, b^{\prime}\right)\right|<\frac{\varepsilon}{4}, \\
\text { for all }\left(b^{\prime}, t\right) \in N_{\delta}(b) \times T .
\end{array}
$$

Now define $s_{i}^{*} \in \mathscr{P}_{i}$ as follows: for each $t_{i} \in T_{i}$,

$$
s_{i}^{*}\left(t_{i}\right):= \begin{cases}\alpha\left(t_{i}\right) s_{i}\left(t_{i}\right)+\left(1-\alpha\left(t_{i}\right)\right) \bar{b} & \text { if } f_{i}\left(t,\left(s_{i}\left(t_{i}\right), b_{-i}\right)\right) \geq g_{i}\left(t,\left(s_{i}\left(t_{i}\right), b_{-i}\right)\right) \\ & \text { for some }\left(t_{-i}, b_{-i}\right) \in T_{-i} \times B_{-i}, \\ \beta\left(t_{i}\right) s_{i}\left(t_{i}\right)+\left(1-\beta\left(t_{i}\right)\right) \underline{b} & \text { if } f_{i}\left(t,\left(s_{i}\left(t_{i}\right), b_{-i}\right)\right)<g_{i}\left(t,\left(s_{i}\left(t_{i}\right), b_{-i}\right)\right) \\ & \text { for some }\left(t_{-i}, b_{-i}\right) \in T_{-i} \times B_{-i},\end{cases}
$$

where $\alpha$ and $\beta$ are $\left(\mathscr{T}_{i}, \mathscr{B}((0,1))\right)$-measurable maps from $T_{i}$ to $(0,1)$ such that $\alpha\left(t_{i}\right) s_{i}\left(t_{i}\right)+(1-$ $\left.\alpha\left(t_{i}\right)\right) \bar{b}$ and $\beta\left(t_{i}\right) s_{i}\left(t_{i}\right)+\left(1-\beta\left(t_{i}\right)\right) \underline{b}$ belong to $N_{\delta}\left(s_{i}\left(t_{i}\right)\right)$ for each $t_{i} \in T_{i} .{ }^{19}$ Note that Assumption C(ii) ensures that $s_{i}^{*}$ is well-defined.

Fix $\left(t, b_{-i}\right) \in T \times B_{-i}$ and consider the following cases.

Case 1. $s_{i}\left(t_{i}\right)<\max _{j \neq i} b_{j}$. If $f_{i}\left(t,\left(s_{i}\left(t_{i}\right), b_{-i}\right)\right) \geq g_{i}\left(t,\left(s_{i}\left(t_{i}\right), b_{-i}\right)\right)$, then, using (17), we have, for $b_{-i}^{\prime} \in N_{\delta}\left(b_{-i}\right)$,

$$
f_{i}\left(t,\left(s_{i}^{*}\left(t_{i}\right), b_{-i}^{\prime}\right)\right) \geq f_{i}\left(t,\left(s_{i}\left(t_{i}\right), b_{-i}\right)\right)-\frac{\varepsilon}{4} \geq g_{i}\left(t,\left(s_{i}\left(t_{i}\right), b_{-i}\right)\right)-\frac{\varepsilon}{4} \geq g_{i}\left(t,\left(s_{i}^{*}\left(t_{i}\right), b_{-i}^{\prime}\right)\right)-\frac{\varepsilon}{2},
$$

and so

$$
\begin{array}{rlr}
u_{i}\left(t,\left(s_{i}^{*}\left(t_{i}\right), b_{-i}^{\prime}\right)\right) & \geq g_{i}\left(t,\left(s_{i}^{*}\left(t_{i}\right), b_{-i}^{\prime}\right)\right)+h_{i}\left(t,\left(s_{i}^{*}\left(t_{i}\right), b_{-i}^{\prime}\right)\right)-\frac{\varepsilon}{2} & (\text { by }(18)) \\
& >g_{i}\left(t,\left(s_{i}\left(t_{i}\right), b_{-i}\right)\right)+h_{i}\left(t,\left(s_{i}\left(t_{i}\right), b_{-i}\right)\right)-\varepsilon & \\
& =u_{i}\left(t,\left(s_{i}\left(t_{i}\right), b_{-i}\right)\right)-\varepsilon & \left(\text { since } s_{i}\left(t_{i}\right)<\max _{j \neq i} b_{j}\right) .
\end{array}
$$

If $f_{i}\left(t,\left(s_{i}\left(t_{i}\right), b_{-i}\right)\right)<g_{i}\left(t,\left(s_{i}\left(t_{i}\right), b_{-i}\right)\right)$, then, letting $V_{-i}$ be a neighborhood of $b_{-i}$ with

$$
s_{i}^{*}\left(t_{i}\right)<\max _{j \neq i} b_{j}^{\prime}, \quad \text { for all } b_{-i}^{\prime} \in V_{-i},
$$

and for $b_{-i}^{\prime} \in N_{\delta}\left(b_{-i}\right) \cap V_{-i}$, we have

$$
u_{i}\left(t,\left(s_{i}^{*}\left(t_{i}\right), b_{-i}^{\prime}\right)\right)=g_{i}\left(t,\left(s_{i}^{*}\left(t_{i}\right), b_{-i}^{\prime}\right)\right)+h_{i}\left(t,\left(s_{i}^{*}\left(t_{i}\right), b_{-i}^{\prime}\right)\right)
$$

\footnotetext{
${ }^{19}$ For example, for each $z \in[\underline{b}, \bar{b}]$ let $\lambda(z)=\frac{1}{2}$ if $\bar{b} \leq z+\delta$ and $\lambda(z)=1-\frac{\delta}{2(\bar{b}-z)}$ if $\bar{b} \geq z+\delta$. Then $\lambda:[\underline{b}, \bar{b}] \rightarrow(0,1)$ is continuous and the function $t_{i} \mapsto \alpha\left(t_{i}\right):=\lambda\left(s_{i}\left(t_{i}\right)\right)$ is a $\left(\mathscr{T}_{i}, \mathscr{B}((0,1))\right)$-measurable map with the required property.
} 


$$
\begin{aligned}
& >g_{i}\left(t,\left(s_{i}\left(t_{i}\right), b_{-i}\right)\right)+h_{i}\left(t,\left(s_{i}\left(t_{i}\right), b_{-i}\right)\right)-\varepsilon \\
& =u_{i}\left(t,\left(s_{i}\left(t_{i}\right), b_{-i}\right)\right)-\varepsilon
\end{aligned}
$$

( since $\left.s_{i}\left(t_{i}\right)<\max _{j \neq i} b_{j}\right)$.

Case 2. $s_{i}\left(t_{i}\right)>\max _{j \neq i} b_{j}$. If $f_{i}\left(t,\left(s_{i}\left(t_{i}\right), b_{-i}\right)\right) \geq g_{i}\left(t,\left(s_{i}\left(t_{i}\right), b_{-i}\right)\right)$, then, for $V_{-i}$ a neighborhood of $b_{-i}$ such that

$$
s_{i}^{*}\left(t_{i}\right)>\max _{j \neq i} b_{j}^{\prime}, \quad \text { for all } b_{-i}^{\prime} \in V_{-i},
$$

and for $b_{-i}^{\prime} \in N_{\delta}\left(b_{-i}\right) \cap V_{-i}$,

$$
\begin{aligned}
u_{i}\left(t,\left(s_{i}^{*}\left(t_{i}\right), b_{-i}^{\prime}\right)\right) & =f_{i}\left(t,\left(s_{i}^{*}\left(t_{i}\right), b_{-i}^{\prime}\right)\right)+h_{i}\left(t,\left(s_{i}^{*}\left(t_{i}\right), b_{-i}^{\prime}\right)\right) \\
& >f_{i}\left(t,\left(s_{i}\left(t_{i}\right), b_{-i}\right)\right)+h_{i}\left(t,\left(s_{i}\left(t_{i}\right), b_{-i}\right)\right)-\varepsilon \\
& =u_{i}\left(t,\left(s_{i}\left(t_{i}\right), b_{-i}\right)\right)-\varepsilon
\end{aligned}
$$

( since $\left.s_{i}\left(t_{i}\right)>\max _{j \neq i} b_{j}\right)$.

If $f_{i}\left(t,\left(s_{i}\left(t_{i}\right), b_{-i}\right)\right)<g_{i}\left(t,\left(s_{i}\left(t_{i}\right), b_{-i}\right)\right)$, then, for $b_{-i}^{\prime} \in N_{\delta}\left(b_{-i}\right)$,

$$
g_{i}\left(t,\left(s_{i}^{*}\left(t_{i}\right), b_{-i}^{\prime}\right)\right) \geq g_{i}\left(t,\left(s_{i}\left(t_{i}\right), b_{-i}\right)\right)-\frac{\varepsilon}{4}>f_{i}\left(t,\left(s_{i}\left(t_{i}\right), b_{-i}\right)\right)-\frac{\varepsilon}{4} \geq f_{i}\left(t,\left(s_{i}^{*}\left(t_{i}\right), b_{-i}^{\prime}\right)\right)-\frac{\varepsilon}{2} .
$$

Consequently, for $b_{-i}^{\prime} \in N_{\delta}\left(b_{-i}\right)$,

$$
\begin{array}{rlrl}
u_{i}\left(t,\left(s_{i}^{*}\left(t_{i}\right), b_{-i}^{\prime}\right)\right) & \geq f_{i}\left(t,\left(s_{i}^{*}\left(t_{i}\right), b_{-i}^{\prime}\right)\right)+h_{i}\left(t,\left(s_{i}^{*}\left(t_{i}\right), b_{-i}^{\prime}\right)\right)-\frac{\varepsilon}{2} & (\text { by }(21)) \\
& >f_{i}\left(t,\left(s_{i}\left(t_{i}\right), b_{-i}\right)\right)+h_{i}\left(t,\left(s_{i}\left(t_{i}\right), b_{-i}\right)\right)-\varepsilon & & (\text { by }(17)) \\
& =u_{i}\left(t,\left(s_{i}\left(t_{i}\right), b_{-i}\right)\right)-\varepsilon & & \left(\text { since } s_{i}\left(t_{i}\right)>\max _{j \neq i} b_{j}\right) .
\end{array}
$$

Case 3. $s_{i}\left(t_{i}\right)=\max _{j \neq i} b_{j}$. If $f_{i}\left(t,\left(s_{i}\left(t_{i}\right), b_{-i}\right)\right) \geq g_{i}\left(t,\left(s_{i}\left(t_{i}\right), b_{-i}\right)\right)$, then, for $b_{-i}^{\prime} \in N_{\delta}\left(b_{-i}\right)$, (18) holds. Hence, if $s_{i}\left(t_{i}\right)<\bar{b}$, then, for $V_{-i}$ a neighborhood of $b_{-i}$ such that

$$
s_{i}^{*}\left(t_{i}\right)>\max _{j \neq i} b_{j}^{\prime}, \quad \text { for all } b_{-i}^{\prime} \in V_{-i},
$$

and for $b_{-i}^{\prime} \in N_{\delta}\left(b_{-i}\right) \cap V_{-i}$,

$u_{i}\left(t,\left(s_{i}^{*}\left(t_{i}\right), b_{-i}^{\prime}\right)\right)$

$=f_{i}\left(t,\left(s_{i}^{*}\left(t_{i}\right), b_{-i}^{\prime}\right)\right)+h_{i}\left(t,\left(s_{i}^{*}\left(t_{i}\right), b_{-i}^{\prime}\right)\right)$

$\geq \frac{f_{i}\left(t,\left(s_{i}^{*}\left(t_{i}\right), b_{-i}^{\prime}\right)\right)}{\#\left\{j: b_{j}=s_{i}\left(t_{i}\right)\right\}+1}+\left(1-\frac{1}{\#\left\{j: b_{j}=s_{i}\left(t_{i}\right)\right\}+1}\right) g_{i}\left(t,\left(s_{i}^{*}\left(t_{i}\right), b_{-i}^{\prime}\right)\right)$

$$
\begin{aligned}
>\frac{f_{i}\left(t,\left(s_{i}\left(t_{i}\right), b_{-i}\right)\right)}{\#\left\{j: b_{j}=s_{i}\left(t_{i}\right)\right\}+1}+\left(1-\frac{1}{\#\left\{j: b_{j}=s_{i}\left(t_{i}\right)\right\}+1}\right) & +h_{i}\left(t,\left(s_{i}^{*}\left(t_{i}\right), b_{-i}^{\prime}\right)\right)-\frac{\varepsilon}{2} \\
& +h_{i}\left(t,\left(s_{i}\left(t_{i}\right), b_{-i}\right)\right)
\end{aligned}
$$

( since $\left.s_{i}\left(t_{i}\right)=\max _{j \neq i} b_{j}\right)$.

If, on the other hand, $s_{i}\left(t_{i}\right)=\bar{b}$, then, letting $V_{-i}$ be a neighborhood of $b_{-i}$ such that

$$
\#\left\{j: b_{j}^{\prime}=s_{i}\left(t_{i}\right)\right\}=\#\left\{j: b_{j}^{\prime}=\bar{b}\right\} \leq \#\left\{j: b_{j}=\bar{b}\right\}=\#\left\{j: b_{j}=s_{i}\left(t_{i}\right)\right\}, \quad \text { for all } b_{-i}^{\prime} \in V_{-i},
$$

we have, for $b_{-i}^{\prime} \in N_{\delta}\left(b_{-i}\right) \cap V_{-i}$,

$u_{i}\left(t,\left(s_{i}^{*}\left(t_{i}\right), b_{-i}^{\prime}\right)\right)$ 


$$
\begin{aligned}
& =u_{i}\left(t,\left(s_{i}\left(t_{i}\right), b_{-i}^{\prime}\right)\right) \\
& =\frac{f_{i}\left(t,\left(s_{i}\left(t_{i}\right), b_{-i}^{\prime}\right)\right)}{\#\left\{j: b_{j}^{\prime}=s_{i}\left(t_{i}\right)\right\}+1}+\left(1-\frac{1}{\#\left\{j: b_{j}^{\prime}=s_{i}\left(t_{i}\right)\right\}+1}\right) g_{i}\left(t,\left(s_{i}\left(t_{i}\right), b_{-i}^{\prime}\right)\right) \\
& +h_{i}\left(t,\left(s_{i}\left(t_{i}\right), b_{-i}^{\prime}\right)\right) \\
& >\frac{f_{i}\left(t,\left(s_{i}\left(t_{i}\right), b_{-i}\right)\right)}{\#\left\{j: b_{j}^{\prime}=s_{i}\left(t_{i}\right)\right\}+1}+\left(1-\frac{1}{\#\left\{j: b_{j}^{\prime}=s_{i}\left(t_{i}\right)\right\}+1}\right) g_{i}\left(t,\left(s_{i}\left(t_{i}\right), b_{-i}\right)\right) \\
& +h_{i}\left(t,\left(s_{i}\left(t_{i}\right), b_{-i}\right)\right)-\varepsilon \\
& \geq \frac{f_{i}\left(t,\left(s_{i}\left(t_{i}\right), b_{-i}\right)\right)}{\#\left\{j: b_{j}=s_{i}\left(t_{i}\right)\right\}+1}+\left(1-\frac{1}{\#\left\{j: b_{j}=s_{i}\left(t_{i}\right)\right\}+1}\right) g_{i}\left(t,\left(s_{i}\left(t_{i}\right), b_{-i}\right)\right) \\
& +h_{i}\left(t,\left(s_{i}\left(t_{i}\right), b_{-i}\right)\right)-\varepsilon \\
& \text { (by (23) and } f_{i}\left(t,\left(s_{i}\left(t_{i}\right), b_{-i}\right)\right) \\
& \left.\geq g_{i}\left(t,\left(s_{i}\left(t_{i}\right), b_{-i}\right)\right)\right) \\
& =u_{i}\left(t,\left(s_{i}\left(t_{i}\right), b_{-i}\right)\right)-\varepsilon
\end{aligned}
$$

If $f_{i}\left(t,\left(s_{i}\left(t_{i}\right), b_{-i}\right)\right)<g_{i}\left(t,\left(s_{i}\left(t_{i}\right), b_{-i}\right)\right)$, then (21) holds for all $b_{-i}^{\prime} \in N_{\delta}\left(b_{-i}\right)$. Therefore, if $s_{i}\left(t_{i}\right)>\underline{b}$, then for $V_{-i}$ a neighborhood of $b_{-i}$ such that

$$
s_{i}^{*}\left(t_{i}\right)<\max _{j \neq i} b_{j}^{\prime}, \quad \text { for all } b_{-i}^{\prime} \in V_{-i},
$$

and for $b_{-i}^{\prime} \in N_{\delta}\left(b_{-i}\right) \cap V_{-i}$

$$
\begin{aligned}
u_{i}\left(t,\left(s_{i}^{*}\left(t_{i}\right), b_{-i}^{\prime}\right)\right) & \\
& =g_{i}\left(t,\left(s_{i}^{*}\left(t_{i}\right), b_{-i}^{\prime}\right)\right)+h_{i}\left(t,\left(s_{i}^{*}\left(t_{i}\right), b_{-i}^{\prime}\right)\right) \\
& \geq \frac{f_{i}\left(t,\left(s_{i}^{*}\left(t_{i}\right), b_{-i}^{\prime}\right)\right)}{\#\left\{j: b_{j}=s_{i}\left(t_{i}\right)\right\}+1}+\left(1-\frac{1}{\#\left\{j: b_{j}=s_{i}\left(t_{i}\right)\right\}+1}\right) g_{i}\left(t,\left(s_{i}^{*}\left(t_{i}\right), b_{-i}^{\prime}\right)\right) \\
& +h_{i}\left(t,\left(s_{i}^{*}\left(t_{i}\right), b_{-i}^{\prime}\right)\right)-\frac{\varepsilon}{2} \\
& \frac{f_{i}\left(t,\left(s_{i}\left(t_{i}\right), b_{-i}\right)\right)}{\#\left\{j: b_{j}=s_{i}\left(t_{i}\right)\right\}+1}+\left(1-\frac{1}{\#\left\{j: b_{j}=s_{i}\left(t_{i}\right)\right\}+1}\right) g_{i}\left(t,\left(s_{i}\left(t_{i}\right), b_{-i}\right)\right) \\
& +h_{i}\left(t,\left(s_{i}\left(t_{i}\right), b_{-i}\right)\right)-\varepsilon
\end{aligned}
$$

Now suppose that $s_{i}\left(t_{i}\right)=\underline{b}$. Then $s_{i}\left(t_{i}\right)=\underline{b}=\max _{j \neq i} b_{j}$. Consequently, $b_{j}=\underline{b}$ for all $j \neq i$, and for $b_{-i}^{\prime} \in N_{\delta}\left(b_{-i}\right)$ with $\underline{b}<\max _{j \neq i} b_{j}^{\prime}$ we have

$$
\begin{aligned}
& u_{i}\left(t,\left(s_{i}^{*}\left(t_{i}\right), b_{-i}^{\prime}\right)\right) \\
& =u_{i}\left(t,\left(s_{i}\left(t_{i}\right), b_{-i}^{\prime}\right)\right) \\
& =g_{i}\left(t,\left(s_{i}\left(t_{i}\right), b_{-i}^{\prime}\right)\right)+h_{i}\left(t,\left(s_{i}\left(t_{i}\right), b_{-i}^{\prime}\right)\right) \\
& >g_{i}\left(t,\left(s_{i}\left(t_{i}\right), b_{-i}\right)\right)+h_{i}\left(t,\left(s_{i}\left(t_{i}\right), b_{-i}\right)\right)-\varepsilon \\
& =\frac{g_{i}\left(t,\left(s_{i}\left(t_{i}\right), b_{-i}\right)\right)}{N}+\left(1-\frac{1}{N}\right) g_{i}\left(t,\left(s_{i}\left(t_{i}\right), b_{-i}\right)\right) \\
& +h_{i}\left(t,\left(s_{i}\left(t_{i}\right), b_{-i}\right)\right)-\varepsilon \\
& >\frac{f_{i}\left(t,\left(s_{i}\left(t_{i}\right), b_{-i}\right)\right)}{N}+\left(1-\frac{1}{N}\right) g_{i}\left(t,\left(s_{i}\left(t_{i}\right), b_{-i}\right)\right) \\
& +h_{i}\left(t,\left(s_{i}\left(t_{i}\right), b_{-i}\right)\right)-\varepsilon \quad\left(\text { since } f_{i}\left(t,\left(s_{i}\left(t_{i}\right), b_{-i}\right)\right)<g_{i}\left(t,\left(s_{i}\left(t_{i}\right), b_{-i}\right)\right)\right)
\end{aligned}
$$




$$
=u_{i}\left(t,\left(s_{i}\left(t_{i}\right), b_{-i}\right)\right)-\varepsilon
$$

(since $\left.\underline{b}=s_{i}\left(t_{i}\right)=\max _{j \neq i} b_{j}\right)$

and for $b_{-i}^{\prime} \in N_{\delta}\left(b_{-i}\right)$ with $\underline{b}=\max _{j \neq i} b_{j}^{\prime}$ we have

$$
s_{i}^{*}\left(t_{i}\right)=s_{i}\left(t_{i}\right)=\underline{b}=b_{j}=b_{j}^{\prime}, \quad \text { for all } j \neq i,
$$

implying that

$$
u_{i}\left(t,\left(s_{i}^{*}\left(t_{i}\right), b_{-i}^{\prime}\right)\right)=u_{i}\left(t,\left(s_{i}\left(t_{i}\right), b_{-i}\right)\right)>u_{i}\left(t,\left(s_{i}\left(t_{i}\right), b_{-i}\right)\right)-\varepsilon .
$$

This establishes uniform payoff security of $\Gamma$.

\section{A.2.2 Proof of Corollary 4}

Corollary 4 (to Theorem 1). Under Assumptions $E-H$, the Cournot game $\Gamma$ defined in (6) possesses a Bayes-Nash equilibrium.

Proof. By Corollary 1 and Remark 7, it suffices to show that under the conditions (i)-(ii) in Remark 7, $\Gamma$ satisfies Condition 1 and for each $t \in T$, the map $\sum_{i=1}^{N} u_{i}(t, \cdot): X \rightarrow \mathbb{R}$ is upper semicontinuous. Since

$$
\sum_{i=1}^{N} u_{i}(t, q)=\left(\sum_{i=1}^{N} q_{i}\right) p\left(t, \sum_{i=1}^{N} q_{i}\right)-\left(\sum_{i=1}^{N} c_{i}\left(t, q_{i}\right)\right),
$$

Assumptions G-H ensure that $\sum_{i=1}^{N} u_{i}(t, \cdot): X \rightarrow \mathbb{R}$ is upper semicontinuous for each $t \in T$. To see that $\Gamma$ satisfies Condition 1 , fix $i$ and $\varepsilon>0$. Define $\phi: X_{i} \rightarrow X_{i}$ by $\phi\left(q_{i}\right):=q_{i}$. The map $\phi$ is clearly $\left(\mathscr{B}\left(X_{i}\right), \mathscr{B}\left(X_{i}\right)\right)$-measurable. We must show that for each $(t, q) \in T \times X$, there is a neighborhood $V_{q_{-i}}$ of $q_{-i}$ such that

$$
u_{i}\left(t,\left(\phi\left(q_{i}\right), x_{-i}\right)\right)>u_{i}\left(t,\left(q_{i}, q_{-i}\right)\right)-\varepsilon, \text { for all } x_{-i} \in V_{q_{-i}} .
$$

Fix $(t, q) \in T \times X$. Since $\left.p(t, \cdot)\right|_{\left\{\sum_{i=1}^{N} q_{i}:\left(q_{1}, \ldots, q_{N}\right) \in X\right\}}$ is lower semicontinuous, there exists a neighborhood $V_{q_{-i}}$ of $q_{-i}$ such that for every $x_{-i} \in V_{q_{-i}}$,

$$
q_{i} p\left(t, q_{i}+\sum_{j \neq i} x_{j}\right)>q_{i} p\left(t, \sum_{j=1}^{N} q_{j}\right)-\frac{\varepsilon}{2} .
$$

In addition, it is clear that

$$
-c_{i}\left(t, q_{i}\right)>-c_{i}\left(t, q_{i}\right)-\frac{\varepsilon}{2} .
$$

For such $V_{q_{-i}}$ we have, for every $x_{-i} \in V_{q_{-i}}$,

$$
\begin{aligned}
u_{i}\left(t,\left(\phi\left(q_{i}\right), x_{-i}\right)\right) & =u_{i}\left(t,\left(q_{i}, x_{-i}\right)\right) \\
& =q_{i} p\left(t, q_{i}+\sum_{j \neq i}^{N} x_{j}\right)-c_{i}\left(t, q_{i}\right) \\
& >q_{i} p\left(t, \sum_{j=1}^{N} q_{j}\right)-c_{i}\left(t, q_{i}\right)-\varepsilon \\
& =u_{i}(t, q)-\varepsilon
\end{aligned}
$$

where the inequality uses (26) and (27). Hence, (25) holds. 


\section{A.2.3 Proof of Corollary 5}

Corollary 5 (to Theorem 1). Under Assumptions I-O, the game $\Gamma$ defined in (8) possesses a Bayes-Nash equilibrium.

Proof. By virtue of Theorem 1, it suffices to show that $\Gamma$ is uniformly payoff secure and the $\operatorname{map} \sum_{i=1}^{N} u_{i}(t, \cdot): X \rightarrow \mathbb{R}$ is upper semicontinuous for each $t \in T$.

We begin by showing that for each $i$ and $\left(t, p, q_{1}, q_{2}\right) \in T \times\left(X_{1} \cap X_{2}\right) \times Y_{1} \times Y_{2}$,

$$
q_{i}<G_{i}\left(t, p, q_{1}, q_{2}\right) \quad \text { and } \quad q_{-i}>G_{-i}\left(t, p, q_{1}, q_{2}\right)
$$

cannot hold simultaneously. Suppose that (28) holds. Then

$\min \left\{q_{i}, G_{i}\left(t, p, q_{1}, q_{2}\right)\right\}+\min \left\{q_{-i}, G_{-i}\left(t, p, q_{1}, q_{2}\right)\right\}=q_{i}+G_{-i}\left(t, p, q_{1}, q_{2}\right)<\min \left\{q_{i}+q_{-i}, D(t, p)\right\}$, implying

$\min \left\{q_{i}, G_{i}\left(t, p, q_{1}, q_{2}\right)\right\}+\min \left\{q_{-i}, G_{-i}\left(t, p, q_{1}, q_{2}\right)\right\}<\min \left\{q_{i}, D(t, p)\right\}+\min \left\{q_{-i}, \max \left\{D(t, p)-q_{i}, 0\right\}\right\}$

and contradicting Assumption $\mathrm{O}$.

For each $t \in T$, the sum

$$
\sum_{i=1}^{2} p_{i} \min \left\{q_{i}, D_{i}\left(t, p_{1}, q_{1}, p_{2}, q_{2}\right)\right\}
$$

is upper semicontinuous on $X_{1} \times Y_{1} \times X_{2} \times Y_{2}$ (this is shown below). Therefore, in light of Assumption $\mathrm{K}$, the sum $\sum_{i=1}^{N} u_{i}(t, \cdot)$ is upper semicontinuous for each $t \in T$. To see that the sum in (29) is upper semicontinuous, note first that Assumptions L and $\mathrm{N}$ imply that for each $t, \sum_{i=1}^{2} p_{i} \min \left\{q_{i}, D_{i}\left(t, p_{1}, q_{1}, p_{2}, q_{2}\right)\right\}$ is continuous at points $\left(p_{1}, q_{1}, p_{2}, q_{2}\right)$ with $p_{1} \neq p_{2}$. Pick $t$ and a profile $\left(p_{1}, q_{1}, p_{2}, q_{2}\right)$ with $p_{1}=p_{2}$. We have seen that

either $\left[q_{1} \leq G_{1}\left(t, p_{1}, q_{1}, q_{2}\right)\right.$ and $\left.q_{2} \leq G_{2}\left(t, p_{1}, q_{1}, q_{2}\right)\right]$

$$
\text { or }\left[q_{1} \geq G_{1}\left(t, p_{1}, q_{1}, q_{2}\right) \text { and } q_{2} \geq G_{2}\left(t, p_{1}, q_{1}, q_{2}\right)\right] \text {. }
$$

Let $\left(p_{1}^{n}, q_{1}^{n}, p_{2}^{n}, q_{2}^{n}\right)$ be a sequence with limit point $\left(p_{1}, q_{1}, p_{2}, q_{2}\right)$. Then, for each $n$,

$$
\begin{aligned}
\sum_{j=1}^{2} p_{j}^{n} \min \left\{q_{j}^{n},\right. & \left.D_{j}\left(t, p_{1}^{n}, q_{1}^{n}, p_{2}^{n}, q_{2}^{n}\right)\right\} \\
& = \begin{cases}p_{1}^{n} \min \left\{q_{1}^{n}, G_{1}\left(t, p_{1}^{n}, q_{1}^{n}, q_{2}^{n}\right)\right\}+p_{2}^{n} \min \left\{q_{2}^{n}, G_{2}\left(t, p_{1}^{n}, q_{1}^{n}, q_{2}^{n}\right)\right\} & \text { if } p_{1}^{n}=p_{2}^{n}, \\
p_{i}^{n} \min \left\{q_{i}^{n}, D\left(t, p_{i}^{n}\right)\right\}+p_{-i}^{n} \min \left\{q_{-i}^{n}, H_{-i}\left(t, p_{1}^{n}, p_{2}^{n}, q_{i}^{n}\right)\right\} & \text { if } p_{i}^{n}<p_{-i}^{n},\end{cases}
\end{aligned}
$$

and, in light of (30), either (i) $q_{1} \geq G_{1}\left(t, p_{1}, q_{1}, q_{2}\right), q_{2} \geq G_{2}\left(t, p_{1}, q_{1}, q_{2}\right)$, and in this case

$$
\sum_{j=1}^{2} p_{j} \min \left\{q_{j}, D_{j}\left(t, p_{1}, q_{1}, p_{2}, q_{2}\right)\right\}=p_{1} D\left(t, p_{1}\right),
$$

or (ii) $q_{1} \leq G_{1}\left(t, p_{1}, q_{1}, q_{2}\right), q_{2} \leq G_{2}\left(t, p_{1}, q_{1}, q_{2}\right)$, whence

$$
\sum_{j=1}^{2} p_{j} \min \left\{q_{j}, D_{j}\left(t, p_{1}, q_{1}, p_{2}, q_{2}\right)\right\}=p_{1}\left(q_{1}+q_{2}\right)
$$

For subsequences $\left(p_{1}^{n_{k}}, q_{1}^{n_{k}}, p_{2}^{n_{k}}, q_{2}^{n_{k}}\right)$ such that $p_{1}^{n_{k}}=p_{2}^{n_{k}}$ for each $k$, we have

$$
\sum_{j=1}^{2} p_{j}^{n_{k}} \min \left\{q_{j}^{n_{k}}, D_{j}\left(t, p_{1}^{n_{k}}, q_{1}^{n_{k}}, p_{2}^{n_{k}}, q_{2}^{n_{k}}\right)\right\} \leq p_{1}^{n_{k}} D\left(t, p_{1}^{n_{k}}\right) .
$$


Therefore, in case (i) we have (by the continuity of $D(t, \cdot)$ )

$\underset{k}{\limsup } \sum_{j=1}^{2} p_{j}^{n_{k}} \min \left\{q_{j}^{n_{k}}, D_{j}\left(t, p_{1}^{n_{k}}, q_{1}^{n_{k}}, p_{2}^{n_{k}}, q_{2}^{n_{k}}\right)\right\} \leq p_{1} D(t, p)=\sum_{j=1}^{2} p_{j} \min \left\{q_{j}, D_{j}\left(t, p_{1}, q_{1}, p_{2}, q_{2}\right)\right\}$, and, in case (ii),

$\underset{k}{\limsup } \sum_{j=1}^{2} p_{j}^{n_{k}} \min \left\{q_{j}^{n_{k}}, D_{j}\left(t, p_{1}^{n_{k}}, q_{1}^{n_{k}}, p_{2}^{n_{k}}, q_{2}^{n_{k}}\right)\right\} \leq p_{1}\left(q_{1}+q_{2}\right)=\sum_{j=1}^{2} p_{j} \min \left\{q_{j}, D_{j}\left(t, p_{1}, q_{1}, p_{2}, q_{2}\right)\right\}$.

For subsequences $\left(p_{1}^{n_{k}}, q_{1}^{n_{k}}, p_{2}^{n_{k}}, q_{2}^{n_{k}}\right)$ such that $p_{i}^{n_{k}}<p_{-i}^{n_{k}}$ for each $k$, we have

$\sum_{j=1}^{2} p_{j}^{n_{k}} \min \left\{q_{j}^{n_{k}}, D_{j}\left(t, p_{1}^{n_{k}}, q_{1}^{n_{k}}, p_{2}^{n_{k}}, q_{2}^{n_{k}}\right)\right\}$

$=p_{i}^{n_{k}} \min \left\{q_{i}^{n_{k}}, D\left(t, p_{i}^{n_{k}}\right)\right\}+p_{-i}^{n_{k}} \min \left\{q_{-i}^{n_{k}}, H_{-i}\left(t, p_{1}^{n_{k}}, p_{2}^{n_{k}}, q_{i}^{n_{k}}\right)\right\}$

$\rightarrow p_{i} \min \left\{q_{i}, D\left(t, p_{i}\right)\right\}+p_{-i} \min \left\{q_{-i}, H_{-i}\left(t, p_{1}, p_{2}, q_{i}\right)\right\}$

(by Assumptions L and N)

$=p_{i} \min \left\{q_{i}, D\left(t, p_{i}\right)\right\}+p_{-i} \min \left\{q_{-i}, \max \left\{0, D\left(t, p_{i}\right)-q_{i}\right\}\right\}$

(by Assumption N)

$\leq p_{i} \min \left\{q_{i}, G_{i}\left(t, p_{i}, q_{1}, q_{2}\right)\right\}+p_{-i} \min \left\{q_{-i}, G_{-i}\left(t, p_{i}, q_{1}, q_{2}\right)\right\} \quad$ (by $p_{i}=p_{-i}$ and Assumption O)

$=\sum_{j=1}^{2} p_{j} \min \left\{q_{j}, D_{j}\left(t, p_{1}, q_{1}, p_{2}, q_{2}\right)\right\}$.

To see that $\Gamma$ is uniformly payoff secure, fix $i, \varepsilon>0$, and $s_{i} \in \mathscr{P}_{i}$. By Assumption L, the family $\{D(t, \cdot): t \in T\}$ is equicontinuous on $\mathbb{R}_{+}$. Since the set $X_{1} \cup X_{2}$ is compact, it follows that $\{D(t, \cdot): t \in T\}$ is uniformly equicontinuous on $X_{1} \cup X_{2}$. Similarly, using Assumption $\mathrm{N}$ and the fact that $X_{1} \times X_{2} \times Y_{-i}$ is compact, we see that $\left\{H_{i}(t, \cdot): t \in T\right\}$ is uniformly equicontinuous on $X_{1} \times X_{2} \times Y_{-i}$. Consequently, there exists $\delta>0$ such that

$$
\begin{gathered}
\forall(p, q) \in \mathbb{R}_{+}^{3}, p^{\prime} \min \left\{q^{\prime}, D\left(t, p^{\prime}\right)\right\}>p \min \{q, D(t, p)\}-\varepsilon \forall\left(p^{\prime}, q^{\prime}, t\right) \in N_{\delta}(p, q) \times T, \\
\forall(p, q, \hat{q}) \in \mathbb{R}_{+}^{3}, \\
p^{\prime} \min \left\{q, \max \left\{D\left(t, p^{\prime}\right)-\hat{q}^{\prime}, 0\right\}\right\} \\
>p \min \{q, \max \{D(t, p)-\hat{q}, 0\}\}-\frac{\varepsilon}{2} \forall\left(p^{\prime}, \hat{q}^{\prime}, t\right) \in N_{\delta}(p, \hat{q}) \times T,
\end{gathered}
$$

and

$$
\begin{array}{r}
\forall(p, q) \in X_{1} \times X_{2} \times Y_{1} \times Y_{2}, \\
p_{i}^{\prime} \min \left\{q_{i}, H_{i}\left(t, p^{\prime}, q_{-i}^{\prime}\right)\right\}>p_{i} \min \left\{q_{i}, H_{i}\left(t, p, q_{-i}\right)\right\}-\frac{\varepsilon}{2} \forall\left(p^{\prime}, q_{-i}^{\prime}, t\right) \in N_{\delta}\left(p, q_{-i}\right) \times T .
\end{array}
$$

Now denote $s_{i}\left(t_{i}\right)$ by $\left(p_{i}\left(t_{i}\right), q_{i}\left(t_{i}\right)\right)$ and define $s_{i}^{*} \in \mathscr{P}_{i}$ as follows:

$$
s_{i}^{*}\left(t_{i}\right):=\left(p_{i}^{*}\left(t_{i}\right), q_{i}\left(t_{i}\right)\right):=\left(\alpha\left(t_{i}\right) p_{i}\left(t_{i}\right), q_{i}\left(t_{i}\right)\right),
$$

where $\alpha(\cdot)$ is a $\left(\mathscr{T}_{i}, \mathscr{B}((0,1))\right)$-measurable map from $T_{i}$ to $(0,1)$ such that $p_{i}^{*}\left(t_{i}\right) \in N_{\delta}\left(p_{i}\left(t_{i}\right)\right)$ for each $t_{i} \in T_{i}$.

Fix $\left(t, p_{-i}, q_{-i}\right) \in T \times X_{-i} \times Y_{-i}$.

Case 1. If $p_{i}^{*}\left(t_{i}\right)>p_{-i}$, then, letting $V_{p_{-i}}$ be a neighborhood of $p_{-i}$ with $p_{i}^{*}\left(t_{i}\right) \notin V_{p_{-i}}$, we have, for $\left(p_{-i}^{\prime}, q_{-i}^{\prime}\right) \in N_{\delta}\left(p_{-i}, q_{-i}\right) \cap\left(V_{p_{-i}} \times Y_{-i}\right)$,

$$
\begin{array}{rlr}
u_{i}\left(t, s_{i}^{*}\left(t_{i}\right), p_{-i}^{\prime}, q_{-i}^{\prime}\right) & =p_{i}^{*}\left(t_{i}\right) \min \left\{q_{i}\left(t_{i}\right), H_{i}\left(t, p_{i}^{*}\left(t_{i}\right), p_{-i}^{\prime}, q_{-i}^{\prime}\right)\right\}-c_{i}\left(t, q_{i}\left(t_{i}\right)\right) & \left(\text { since } p_{i}^{*}\left(t_{i}\right) \notin V_{p_{-i}}\right) \\
& >p_{i}\left(t_{i}\right) \min \left\{q_{i}\left(t_{i}\right), H_{i}\left(t, p_{i}\left(t_{i}\right), p_{-i}, q_{-i}\right)\right\}-c_{i}\left(t, q_{i}\left(t_{i}\right)\right)-\varepsilon & (\text { by }(33)) \\
& =u_{i}\left(t, s_{i}\left(t_{i}\right), p_{-i}, q_{-i}\right)-\varepsilon & \text { (since } p_{i}\left(t_{i}>p_{-i}\right) .
\end{array}
$$


Case 2. If $p_{i}^{*}\left(t_{i}\right)<p_{-i}$, letting $V_{p_{-i}}$ be a neighborhood of $p_{-i}$ such that $p^{*}\left(t_{i}\right) \notin V_{p_{-i}}$, we have, for $\left(p_{-i}^{\prime}, q_{-i}^{\prime}\right) \in N_{\delta}\left(p_{-i}, q_{-i}\right) \cap\left(V_{p_{-i}} \times Y_{-i}\right)$,

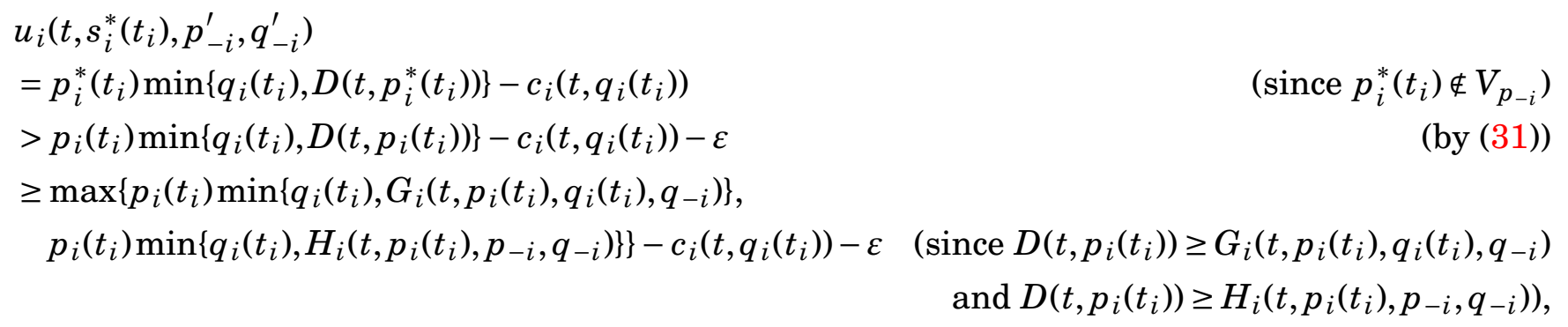

whence $u_{i}\left(t, s_{i}^{*}\left(t_{i}\right), p_{-i}^{\prime}, q_{-i}^{\prime}\right)>u_{i}\left(t, s_{i}\left(t_{i}\right), p_{-i}, q_{-i}\right)-\varepsilon$.

Case 3. $p_{i}^{*}\left(t_{i}\right)=p_{-i}$.

3.1. If $p_{i}^{*}\left(t_{i}\right)<p_{i}\left(t_{i}\right)$, let $V_{p_{-i}}$ be a neighborhood of $p_{-i}$ such that $p_{i}\left(t_{i}\right) \notin V_{p_{-i}}$ and choose $\left(p_{-i}^{\prime}, q_{-i}^{\prime}\right) \in N_{\delta}\left(p_{-i}, q_{-i}\right) \cap\left(V_{p_{-i}} \times Y_{-i}\right)$.

3.1.1. If $p_{i}^{*}\left(t_{i}\right) \neq p_{-i}^{\prime}$, we have

$$
\begin{aligned}
& u_{i}\left(t, s_{i}^{*}\left(t_{i}\right), p_{-i}^{\prime}, q_{-i}^{\prime}\right) \\
& \geq p_{i}^{*}\left(t_{i}\right) \min \left\{q_{i}\left(t_{i}\right), H_{i}\left(t, p_{i}^{*}\left(t_{i}\right), p_{-i}^{\prime}, q_{-i}^{\prime}\right)\right\}-c_{i}\left(t, q_{i}\left(t_{i}\right)\right) \quad\left(\text { since } p_{i}^{*}\left(t_{i}\right) \neq p_{-i}^{\prime} \text { and } D\left(t, p_{i}^{*}\left(t_{i}\right)\right)\right. \\
& >p_{i}\left(t_{i}\right) \min \left\{q_{i}\left(t_{i}\right), H_{i}\left(t, p_{i}\left(t_{i}\right), p_{-i}, q_{-i}\right)\right\}-c_{i}\left(t, q_{i}\left(t_{i}\right)\right)-\varepsilon \\
& =u_{i}\left(t, s_{i}\left(t_{i}\right), p_{-i}, q_{-i}\right)-\varepsilon
\end{aligned}
$$$$
\left.\geq H_{i}\left(t, p_{i}^{*}\left(t_{i}\right), p_{-i}^{\prime}, q_{-i}^{\prime}\right)\right)
$$

(since $\left.p_{i}\left(t_{i}\right)>p_{-i}\right)$;

3.1.2. If $p_{i}^{*}\left(t_{i}\right)=p_{-i}^{\prime}$, we have

$$
\begin{aligned}
& u_{i}\left(t, s_{i}^{*}\left(t_{i}\right), p_{-i}^{\prime}, q_{-i}^{\prime}\right) \\
& \quad=p_{i}^{*}\left(t_{i}\right) \min \left\{q_{i}\left(t_{i}\right), G_{i}\left(t, p_{i}^{*}\left(t_{i}\right), q_{i}\left(t_{i}\right), q_{-i}^{\prime}\right)\right\}-c_{i}\left(t, q_{i}\left(t_{i}\right)\right) \\
& \quad \geq p_{i}^{*}\left(t_{i}\right) \min \left\{q_{i}\left(t_{i}\right), \max \left\{D\left(t, p_{i}^{*}\left(t_{i}\right)\right)-q_{-i}^{\prime}, 0\right\}\right\}-c_{i}\left(t, q_{i}\left(t_{i}\right)\right) \\
& \quad>p_{i}\left(t_{i}\right) \min \left\{q_{i}\left(t_{i}\right), \max \left\{D\left(t, p_{i}\left(t_{i}\right)\right)-q_{-i}, 0\right\}\right\}-c_{i}\left(t, q_{i}\left(t_{i}\right)\right)-\frac{\varepsilon}{2} \\
& \quad=p_{i}\left(t_{i}\right) \min \left\{q_{i}\left(t_{i}\right), H_{i}\left(t, p_{i}\left(t_{i}\right), p_{i}\left(t_{i}\right), q_{-i}\right)\right\}-c_{i}\left(t, q_{i}\left(t_{i}\right)\right)-\frac{\varepsilon}{2} \\
& \quad>p_{i}\left(t_{i}\right) \min \left\{q_{i}\left(t_{i}\right), H_{i}\left(t, p_{i}\left(t_{i}\right), p_{-i}, q_{-i}\right)\right\}-c_{i}\left(t, q_{i}\left(t_{i}\right)\right)-\varepsilon \\
& \quad=u_{i}\left(t, s_{i}\left(t_{i}\right), p_{-i}, q_{-i}\right)-\varepsilon
\end{aligned}
$$

(since $\left.p^{*}\left(t_{i}\right)=p_{-i}^{\prime}\right)$

(by Remark 8)

(by (32))

( since $H_{i}\left(t, p_{i}\left(t_{i}\right), p_{i}\left(t_{i}\right), q_{-i}\right)$

$$
\left.=\max \left\{D\left(t, p_{i}\left(t_{i}\right)\right)-q_{-i}, 0\right\}\right)
$$

(by (33))

(since $\left.p_{i}\left(t_{i}\right)>p_{-i}\right)$.

3.2. If $p_{i}\left(t_{i}\right)=p_{i}^{*}\left(t_{i}\right)=p_{-i}$, then $p_{i}\left(t_{i}\right)=p_{i}^{*}\left(t_{i}\right)=0=p_{-i}$, and in this case, for $\left(p_{-i}^{\prime}, q_{-i}^{\prime}\right) \in$ $N_{\delta}\left(p_{-i}, q_{-i}\right)$ we have

$$
u_{i}\left(t, s_{i}^{*}\left(t_{i}\right), p_{-i}^{\prime}, q_{-i}^{\prime}\right)=-c_{i}\left(t, q_{i}\left(t_{i}\right)\right)>-c_{i}\left(t, q_{i}\left(t_{i}\right)\right)-\varepsilon=u_{i}\left(t, s_{i}\left(t_{i}\right), p_{-i}, q_{-i}\right)-\varepsilon .
$$

This establishes uniform payoff security of $\Gamma$.

\section{A.2.4 Proof of Corollary 6}

Corollary 6 (to Theorem 2). The game $\Gamma$ defined in (10) possesses a Bayes-Nash equilibrium.

We begin with the following lemma. 
Lemma 7. There exists $\varepsilon^{*}>0$ such that the following holds: for each $0<\varepsilon<\varepsilon^{*}$, for each $x_{i} \in\left(0, \alpha_{i}\right]$, and for each $t \in T$, there exists $y_{-i}\left(t, x_{i}\right) \in\left(0, \alpha_{-i}\right]$ such that

$$
\pi_{i}\left(x_{i}, y_{-i}\left(t, x_{i}\right)\right) v(t)=v(t)-\frac{\varepsilon}{4} .
$$

Proof. Choose $0<q<1$ so that

$$
\pi_{i}\left(\alpha_{1}, \alpha_{2}\right)<q, \quad \text { for each } i
$$

and define

$$
\varepsilon^{*}:=4(1-q) \bar{v} \text {. }
$$

Suppose that $0<\varepsilon<\varepsilon^{*}, x_{i} \in\left(0, \alpha_{i}\right]$, and $t \in T$. First, note that $v(t)-\frac{\varepsilon}{4}>\pi_{i}\left(x_{i}, \alpha_{-i}\right) v(t)$ since

$$
\begin{aligned}
v(t)-\frac{\varepsilon}{4}-\pi_{i}\left(x_{i}, \alpha_{-i}\right) v(t) & \geq v(t)-\frac{\varepsilon}{4}-\pi_{i}\left(\alpha_{i}, \alpha_{-i}\right) v(t) \\
& \geq v(t)-\frac{\varepsilon}{4}-q v(t) \\
& =(1-q) v(t)-\frac{\varepsilon}{4} \\
& \geq(1-q) \bar{v}-\frac{\varepsilon}{4} \\
& >0 .
\end{aligned}
$$

Consequently, the result follows from the assumptions that $\pi_{i}\left(x_{i}, \cdot\right)$ is continuous on $\left(0, \alpha_{-i}\right]$ and

$\lim _{x_{-i} \rightarrow 0+} \pi_{i}\left(x_{i}, x_{-i}\right)=1$.

We are now ready to prove the Corollary 6.

Proof of Corollary 6. By Theorem 2, it suffices to show that $\Gamma$ is uniformly diagonally secure. This will be proven as an application of Proposition 2. To accomplish this, choose $\varepsilon^{*}$ as in Lemma 7 and define for each $i$ and for each $0<\varepsilon<\varepsilon^{*}$ the measurable function $\phi_{i}:\left[0, \alpha_{i}\right] \rightarrow\left[0, \alpha_{i}\right]$ by

$$
\phi_{i}\left(d_{i}\right):= \begin{cases}d_{i} & \text { if } d_{i} \in\left(0, \alpha_{i}\right], \\ \frac{\varepsilon}{4} & \text { if } d_{i}=0 .\end{cases}
$$

To apply Proposition 2, we will prove the following: for each $t \in T,\left(d_{1}, d_{2}\right) \in X_{1} \times X_{2}$ and $\varepsilon \in\left(0, \varepsilon^{*}\right)$, the following holds: for each $x=\left(x_{1}, x_{2}\right) \in X_{1} \times X_{2}$, there exists an open set $V$ containing $x$ such that

$$
\begin{aligned}
u_{1}\left(t, \phi_{1}\left(d_{1}\right), w_{2}\right)+u_{2}\left(t, w_{1}, \phi_{2}\left(d_{2}\right)\right) & -u_{1}\left(t, w_{1}, w_{2}\right)-u_{2}\left(t, w_{1}, w_{2}\right) \\
& \geq u_{1}\left(t, d_{1}, x_{2}\right)+u_{2}\left(t, x_{1}, d_{2}\right)-u_{1}\left(t, x_{1}, x_{2}\right)-u_{2}\left(t, x_{1}, x_{2}\right)-\varepsilon
\end{aligned}
$$

for all $\left(w_{1}, w_{2}\right) \in V$. The (tedious) argument is partitioned into different cases. So choose $t \in T,\left(d_{1}, d_{2}\right) \in X_{1} \times X_{2}$, and $\varepsilon \in\left(0, \varepsilon^{*}\right)$. To lighten the notation, we suppress the dependence of each $u_{i}$ on $t$ and will write $v(t)$ simply as $v$. Furthermore, let

$$
F\left(d^{*}, w\right):=u_{1}\left(\phi_{1}\left(d_{1}\right), w_{2}\right)+u_{2}\left(w_{1}, \phi_{2}\left(d_{2}\right)\right)-u_{1}\left(w_{1}, w_{2}\right)-u_{2}\left(w_{1}, w_{2}\right)
$$

and

$$
G(d, x):=u_{1}\left(d_{1}, x_{2}\right)+u_{2}\left(x_{1}, d_{2}\right)-u_{1}\left(x_{1}, x_{2}\right)-u_{2}\left(x_{1}, x_{2}\right) .
$$

Case 1. $d=(0,0)$ so that $\left(\phi_{1}\left(d_{1}\right), \phi_{2}\left(d_{2}\right)\right)=d^{*}=\left(\frac{\varepsilon}{4}, \frac{\varepsilon}{4}\right)$. 
1.1. $x=(0,0)$ implies

$$
G(d, x)=u_{1}(0,0)+u_{2}(0,0)-u_{1}(0,0)-u_{2}(0,0)=0 .
$$

Let $\delta:=\frac{\varepsilon}{4}$ and choose $w \in N_{\delta}(x)$.

1.1.1. $w=(0,0)$.

$$
F\left(d^{*}, w\right)=u_{1}\left(\frac{\varepsilon}{4}, 0\right)+u_{2}\left(0, \frac{\varepsilon}{4}\right)-u_{1}(0,0)-u_{2}(0,0)=2(\mu-\lambda) v-\frac{\varepsilon}{2},
$$

implying that

$$
F\left(d^{*}, w\right)-G(d, x)=2(\mu-\lambda) v-\frac{\varepsilon}{2}-0>-\varepsilon .
$$

1.1.2. $w=\left(w_{1}, 0\right), w_{1}>0$.

$$
F\left(d^{*}, w\right)=u_{1}\left(\frac{\varepsilon}{4}, 0\right)+u_{2}\left(w_{1}, \frac{\varepsilon}{4}\right)-u_{1}\left(w_{1}, 0\right)-u_{2}\left(w_{1}, 0\right)=\pi_{2}\left(w_{1}, \frac{\varepsilon}{4}\right) v-\frac{\varepsilon}{2}+w_{1},
$$

implying that

$$
F\left(d^{*}, w\right)-G(d, x)=\left(\pi_{2}\left(w_{1}, \frac{\varepsilon}{4}\right) v-\frac{\varepsilon}{2}+w_{1}\right)-0>-\varepsilon .
$$

1.1.3. $w=\left(w_{1}, w_{2}\right), w_{1}>0, w_{2}>0$.

$F\left(d^{*}, w\right)$

$=u_{1}\left(\frac{\varepsilon}{4}, w_{2}\right)+u_{2}\left(w_{1}, \frac{\varepsilon}{4}\right)-u_{1}\left(w_{1}, w_{2}\right)-u_{2}\left(w_{1}, w_{2}\right)$

$=\left(\pi_{1}\left(\frac{\varepsilon}{4}, w_{2}\right) v-\frac{\varepsilon}{4}\right)+\left(\pi_{2}\left(w_{1}, \frac{\varepsilon}{4}\right) v-\frac{\varepsilon}{4}\right)-\left(\pi_{1}\left(w_{1}, w_{2}\right) v-w_{1}\right)-\left(\pi_{2}\left(w_{1}, w_{2}\right) v-w_{2}\right)$

$>\left(\pi_{1}\left(w_{1}, w_{2}\right) v-\frac{\varepsilon}{4}\right)+\left(\pi_{2}\left(w_{1}, w_{2}\right) v-\frac{\varepsilon}{4}\right)-\left(\pi_{1}\left(w_{1}, w_{2}\right) v-w_{1}\right)-\left(\pi_{2}\left(w_{1}, w_{2}\right) v-w_{2}\right) \quad\left(\right.$ since $\left.w_{i}<\frac{\varepsilon}{4}\right)$

$=w_{1}+w_{2}-\frac{\varepsilon}{2}$,

implying that

$$
F\left(d^{*}, w\right)-G(d, x)>\left(w_{1}+w_{2}-\frac{\varepsilon}{4}\right)-0>-\varepsilon .
$$

1.2. $x=\left(x_{1}, 0\right), x_{1}>0$ implies

$$
G(d, x)=u_{1}(0,0)+u_{2}\left(x_{1}, 0\right)-u_{1}\left(x_{1}, 0\right)-u_{2}\left(x_{1}, 0\right)=x_{1}+(\lambda-\mu) v .
$$

Let $\delta:=\min \left\{\frac{\varepsilon}{4}, x_{1}\right\}$, choose $w \in N_{\delta}(x)$, and note that $w_{1}>0$.

1.2.1. $w=\left(w_{1}, 0\right), w_{1}>0$.

$$
\begin{aligned}
F\left(d^{*}, w\right) & =u_{1}\left(\frac{\varepsilon}{4}, 0\right)+u_{2}\left(w_{1}, \frac{\varepsilon}{4}\right)-u_{1}\left(w_{1}, 0\right)-u_{2}\left(w_{1}, 0\right) \\
& =\left(\mu v-\frac{\varepsilon}{4}\right)+\left(\pi_{2}\left(w_{1}, \frac{\varepsilon}{4}\right) v-\frac{\varepsilon}{4}\right)-\left(\mu v-w_{1}\right)-0 \\
& =\pi_{2}\left(w_{1}, \frac{\varepsilon}{4}\right) v-\frac{\varepsilon}{2}+w_{1}
\end{aligned}
$$

impying that

$$
\begin{aligned}
F\left(d^{*}, w\right)-G(d, x) & =\pi_{2}\left(w_{1}, \frac{\varepsilon}{4}\right) v-\frac{\varepsilon}{2}+(\mu-\lambda) v+\left(w_{1}-x_{1}\right) \\
& >\pi_{2}\left(w_{1}, \frac{\varepsilon}{4}\right) v-\frac{\varepsilon}{2}+(\mu-\lambda) v-\frac{\varepsilon}{4} \\
& >-\varepsilon
\end{aligned}
$$


1.2.2. $w=\left(w_{1}, w_{2}\right), w_{1}>0, w_{2}>0$.

$F\left(d^{*}, w\right)$

$=u_{1}\left(\frac{\varepsilon}{4}, w_{2}\right)+u_{2}\left(w_{1}, \frac{\varepsilon}{4}\right)-u_{1}\left(w_{1}, w_{2}\right)-u_{2}\left(w_{1}, w_{2}\right)$

$=\left(\pi_{1}\left(\frac{\varepsilon}{4}, w_{2}\right) v-\frac{\varepsilon}{4}\right)+\left(\pi_{2}\left(w_{1}, \frac{\varepsilon}{4}\right) v-\frac{\varepsilon}{4}\right)-\left(\pi_{1}\left(w_{1}, w_{2}\right) v-w_{1}\right)-\left(\pi_{2}\left(w_{1}, w_{2}\right) v-w_{2}\right)$

$>\left(\pi_{1}\left(w_{1}, w_{2}\right) v-\frac{\varepsilon}{4}\right)+\left(\pi_{2}\left(w_{1}, w_{2}\right) v-\frac{\varepsilon}{4}\right)-\left(\pi_{1}\left(w_{1}, w_{2}\right) v-w_{1}\right)-\left(\pi_{2}\left(w_{1}, w_{2}\right) v-w_{2}\right) \quad\left(\right.$ since $\left.w_{i}<\frac{\varepsilon}{4}\right)$

$=w_{1}+w_{2}-\frac{\varepsilon}{2}$

implying that

$$
F\left(d^{*}, w\right)-G(d, x)=(\mu-\lambda) v+w_{2}-\frac{\varepsilon}{2}+\left(w_{1}-x_{1}\right)>(\mu-\lambda) v+w_{2}-\frac{\varepsilon}{2}-\frac{\varepsilon}{4}>-\varepsilon .
$$

1.3. $x=\left(x_{1}, x_{2}\right), x_{1}>0, x_{2}>0$ implies

$$
G(d, x)=u_{1}\left(0, x_{2}\right)+u_{2}\left(x_{1}, 0\right)-u_{1}\left(x_{1}, x_{2}\right)-u_{2}\left(x_{1}, x_{2}\right)=-\left(v-x_{1}-x_{2}\right) .
$$

Let $\delta:=\min \left\{\frac{\varepsilon}{4}, x_{1}, x_{2}\right\}$, choose $w \in N_{\delta}(x)$, and note that $w_{i}>0$ for each $i$. Therefore,

$$
F\left(d^{*}, w\right)=\left(\pi_{1}\left(\frac{\varepsilon}{4}, w_{2}\right) v-\frac{\varepsilon}{4}\right)+\left(\pi_{2}\left(w_{1}, \frac{\varepsilon}{4}\right) v-\frac{\varepsilon}{4}\right)-\left(v-w_{1}-w_{2}\right),
$$

imolying that

$$
\begin{aligned}
F\left(d^{*}, w\right)-G(d, x) & =\pi_{1}\left(\frac{\varepsilon}{4}, w_{2}\right) v+\pi_{2}\left(w_{1}, \frac{\varepsilon}{4}\right) v-\frac{\varepsilon}{2}+\left(w_{1}-x_{1}\right)+\left(w_{2}-x_{2}\right) \\
& >\pi_{1}\left(\frac{\varepsilon}{4}, w_{2}\right) v+\pi_{2}\left(w_{1}, \frac{\varepsilon}{4}\right) v-\frac{\varepsilon}{2}-\frac{\varepsilon}{4}-\frac{\varepsilon}{4} \\
& >-\varepsilon
\end{aligned}
$$

Case 2. $d=\left(d_{1}, 0\right), d_{1}>0$ so that $\left(\phi_{1}\left(d_{1}\right), \phi_{2}\left(d_{2}\right)\right)=d^{*}=\left(d_{1}, \frac{\varepsilon}{4}\right)$.

2.1. $x=(0,0)$ implies

$$
G(d, x)=u_{1}\left(d_{1}, 0\right)+u_{2}(0,0)-u_{1}(0,0)-u_{2}(0,0)=(\mu-\lambda) v-d_{1}
$$

Let $\delta:=\min \left\{\frac{\varepsilon}{4}, y_{1}\left(t, \frac{\varepsilon}{4}\right), y_{2}\left(t, d_{1}\right)\right\}$ and choose $w \in N_{\delta}(x)$.

2.1.1. $w=(0,0)$.

$$
F\left(d^{*}, w\right)=u_{1}\left(d_{1}, 0\right)+u_{2}\left(0, \frac{\varepsilon}{4}\right)-u_{1}(0,0)-u_{2}(0,0)=2(\mu-\lambda) v-d_{1}-\frac{\varepsilon}{4}
$$

Therefore,

$$
F\left(d^{*}, w\right)-G(d, x)=(\mu-\lambda) v-\frac{\varepsilon}{4}>-\varepsilon .
$$

2.1.2. $w=\left(w_{1}, 0\right), w_{1}>0$.

$$
\begin{aligned}
F\left(d^{*}, w\right) & =u_{1}\left(d_{1}, 0\right)+u_{2}\left(w_{1}, \frac{\varepsilon}{4}\right)-u_{1}\left(w_{1}, 0\right)-u_{2}\left(w_{1}, 0\right) \\
& =\pi_{2}\left(w_{1}, \frac{\varepsilon}{4}\right) v-d_{1}+w_{1}-\frac{\varepsilon}{4} \\
& \left.>\pi_{2}\left(y_{1}\left(t, \frac{\varepsilon}{4}\right), \frac{\varepsilon}{4}\right) v-d_{1}+w_{1}-\frac{\varepsilon}{4} \quad \quad \text { (since } w_{1}=\left|w_{1}-x_{1}\right|<y_{1}\left(t, \frac{\varepsilon}{4}\right)\right) \\
& =v-\frac{\varepsilon}{4}-d_{1}+w_{1}-\frac{\varepsilon}{4} .
\end{aligned}
$$


Note that since $1 \geq 2(\mu-\lambda)$,

$$
F\left(d^{*}, w\right)-G(d, x)>\left(v-\frac{\varepsilon}{2}-d_{1}+w_{1}\right)-\left((\mu-\lambda) v-d_{1}\right)=(1+\lambda-\mu) v+w_{1}-\frac{\varepsilon}{2}>-\frac{\varepsilon}{2} .
$$

2.1.3. $w=\left(0, w_{2}\right), w_{2}>0$.

$$
\begin{aligned}
F\left(d^{*}, w\right) & =u_{1}\left(d_{1}, w_{2}\right)+u_{2}\left(0, \frac{\varepsilon}{4}\right)-u_{1}\left(0, w_{2}\right)-u_{2}\left(0, w_{2}\right) \\
& =\left(\pi_{1}\left(d_{1}, w_{2}\right) v-d_{1}\right)+\left(\mu v-\frac{\varepsilon}{4}\right)-0-\left(\mu v-w_{2}\right) \\
& >\left(\pi_{1}\left(d_{1}, y_{2}\left(t, d_{1}\right)\right) v-d_{1}\right)+\left(\mu v-\frac{\varepsilon}{4}\right)-\left(\mu v-w_{2}\right) \quad\left(\text { since } w_{2}=\left|w_{2}-x_{2}\right|<y_{2}\left(t, d_{1}\right)\right) \\
& =\left(v-\frac{\varepsilon}{4}-d_{1}\right)-\frac{\varepsilon}{4}+w_{2},
\end{aligned}
$$

implying that

$$
F\left(d^{*}, w\right)-G(d, x)>\left(v-d_{1}-\frac{\varepsilon}{2}+w_{2}\right)-\left((\mu-\lambda) v-d_{1}\right)=(1+\lambda-\mu) v+w_{2}-\frac{\varepsilon}{2}>-\varepsilon .
$$

2.1.4. $w=\left(w_{1}, w_{2}\right), w_{1}>0, w_{2}>0$. Note that $w_{1}>0$.

$F\left(d^{*}, w\right)$

$$
\begin{aligned}
& =u_{1}\left(d_{1}, w_{2}\right)+u_{2}\left(w_{1}, \frac{\varepsilon}{4}\right)-u_{1}\left(w_{1}, w_{2}\right)-u_{2}\left(w_{1}, w_{2}\right) \\
& =\left(\pi_{1}\left(d_{1}, w_{2}\right) v-d_{1}\right)+\left(\pi_{2}\left(w_{1}, \frac{\varepsilon}{4}\right) v-\frac{\varepsilon}{4}\right)-\left(\pi_{1}\left(w_{1}, w_{2}\right) v-w_{1}\right)-\left(\pi_{2}\left(w_{1}, w_{2}\right) v-w_{2}\right) \\
& >\left(\pi_{1}\left(w_{1}, y_{2}\left(t, d_{1}\right)\right) v-d_{1}\right)+\left(\pi_{2}\left(y_{1}\left(t, \frac{\varepsilon}{4}\right), w_{2}\right) v-\frac{\varepsilon}{4}\right)-\left(\pi_{1}\left(w_{1}, w_{2}\right) v-w_{1}\right)-\left(\pi_{2}\left(w_{1}, w_{2}\right) v-w_{2}\right) \\
& =\left(v-\frac{\varepsilon}{4}-d_{1}\right)+\left(v-\frac{\varepsilon}{4}-\frac{\varepsilon}{4}\right)-\left(\pi_{1}\left(w_{1}, w_{2}\right) v-w_{1}\right)-\left(\pi_{2}\left(w_{1}, w_{2}\right) v-w_{2}\right) \\
& =v-\frac{3 \varepsilon}{4}-d_{1}+w_{1}+w_{2},
\end{aligned}
$$

implying that

$F\left(d^{*}, w\right)-G(d, x)=\left(v-\frac{3 \varepsilon}{4}-d_{1}+w_{1}+w_{2}\right)-\left((\mu-\lambda) v-d_{1}\right)=(1+\lambda-\mu) v-\frac{3 \varepsilon}{4}+w_{1}+w_{2}>-\varepsilon$

2.2. $x=\left(x_{1}, 0\right), x_{1}>0$ implies

$$
G(d, x)=u_{1}\left(d_{1}, 0\right)+u_{2}\left(x_{1}, 0\right)-u_{1}\left(x_{1}, 0\right)-u_{2}\left(x_{1}, 0\right)=\left(\mu v-d_{1}\right)-\left(\mu v-x_{1}\right)=x_{1}-d_{1} .
$$

Let $\delta:=\min \left\{\frac{\varepsilon}{4}, x_{1}, y_{2}\left(t, d_{1}\right)\right\}$ and choose $w \in N_{\delta}(x)$. Note that $w_{1}>0$.

2.2.1. $\left(w_{1}, w_{2}\right)=\left(w_{1}, 0\right), w_{1}>0$.

$$
\begin{aligned}
F\left(d^{*}, w\right) & =u_{1}\left(d_{1}, 0\right)+u_{2}\left(w_{1}, \frac{\varepsilon}{4}\right)-u_{1}\left(w_{1}, 0\right)-u_{2}\left(w_{1}, 0\right) \\
& =\left(\mu v-d_{1}\right)+\left(\pi_{2}\left(w_{1}, \frac{\varepsilon}{4}\right) v-\frac{\varepsilon}{4}\right)-\left(\mu v-w_{1}\right)-0 \\
& =\pi_{2}\left(w_{1}, \frac{\varepsilon}{4}\right) v-\frac{\varepsilon}{4}+w_{1}-d_{1}
\end{aligned}
$$

implying that

$$
\begin{aligned}
F\left(d^{*}, w\right)-G(d, x) & =\left(\pi_{2}\left(w_{1}, \frac{\varepsilon}{4}\right) v-\frac{\varepsilon}{4}+w_{1}-d_{1}\right)-\left(x_{1}-d_{1}\right) \\
& =\pi_{2}\left(w_{1}, \frac{\varepsilon}{4}\right) v-\frac{\varepsilon}{4}+\left(w_{1}-x_{1}\right) \\
& \left.>\pi_{2}\left(w_{1}, \frac{\varepsilon}{4}\right) v-\frac{\varepsilon}{4}-\frac{\varepsilon}{4} \quad \quad \text { (since }\left|w_{1}-x_{1}\right|<\frac{\varepsilon}{4}\right) \\
& >-\varepsilon .
\end{aligned}
$$


2.2.2. $w=\left(w_{1}, w_{2}\right), w_{1}>0, w_{2}>0$.

$F\left(d^{*}, w\right)$

$=u_{1}\left(d_{1}, w_{2}\right)+u_{2}\left(w_{1}, \frac{\varepsilon}{4}\right)-u_{1}\left(w_{1}, w_{2}\right)-u_{2}\left(w_{1}, w_{2}\right)$

$=\left(\pi_{1}\left(d_{1}, w_{2}\right) v-d_{1}\right)+\left(\pi_{2}\left(w_{1}, \frac{\varepsilon}{4}\right) v-\frac{\varepsilon}{4}\right)$

$-\left(\pi_{1}\left(w_{1}, w_{2}\right) v-w_{1}\right)-\left(\pi_{2}\left(w_{1}, w_{2}\right) v-w_{2}\right)$

$>\left(\pi_{1}\left(d_{1}, y_{2}\left(t, d_{1}\right)\right) v-d_{1}\right)+\left(\pi_{2}\left(w_{1}, \frac{\varepsilon}{4}\right) v-\frac{\varepsilon}{4}\right)$

$$
-\left(\pi_{1}\left(w_{1}, w_{2}\right) v-w_{1}\right)-\left(\pi_{2}\left(w_{1}, w_{2}\right) v-w_{2}\right)
$$

(since $\left.w_{2}=\left|w_{2}-x_{2}\right|<y_{2}\left(t, d_{1}\right)\right)$

$=\left(1-\left(\pi_{1}\left(w_{1}, w_{2}\right)\right) v-\frac{\varepsilon}{2}-d_{1}+w_{1}+w_{2}+\pi_{2}\left(w_{1}, \frac{\varepsilon}{4}\right) v-\pi_{2}\left(w_{1}, w_{2}\right) v\right.$

$>-\frac{\varepsilon}{2}-d_{1}+w_{1}+w_{2}$

$\left(\right.$ since $\left.w_{2}<\frac{\varepsilon}{4}\right)$

implying that

$$
\begin{aligned}
F\left(d^{*}, w\right)-G(d, x) & >\left(-\frac{\varepsilon}{2}-d_{1}+w_{1}+w_{2}\right)-\left(x_{1}-d_{1}\right) \\
& =-\frac{\varepsilon}{2}+w_{2}+\left(w_{1}-x_{1}\right) \\
& >-\frac{\varepsilon}{2}+w_{2}-\frac{\varepsilon}{4} \\
& >-\varepsilon
\end{aligned}
$$

(since $\left.\left|w_{1}-x_{1}\right|<\frac{\varepsilon}{4}\right)$

2.3. $x=\left(0, x_{2}\right), x_{2}>0$ implies

$$
G(d, x)=u_{1}\left(d_{1}, x_{2}\right)+u_{2}(0,0)-u_{1}\left(0, x_{2}\right)-u_{2}\left(0, x_{2}\right)=u_{1}\left(d_{1}, x_{2}\right)+(\lambda-\mu) v+x_{2} .
$$

Choose $0<\gamma<\frac{x_{2}}{2}$ so that $u_{1}\left(d_{1}, w_{2}\right)-u_{1}\left(d_{1}, x_{2}\right)>-\frac{\varepsilon}{4}$ for all $w_{2} \in\left(x_{2}-\gamma, x_{2}+\gamma\right) \cap\left[0, \alpha_{2}\right]$. Let $\delta:=\min \left\{\frac{\varepsilon}{4}, \gamma, y_{1}\left(t, \frac{\varepsilon}{4}\right)\right\}$ and choose $w \in N_{\delta}(x)$. Note that $w_{2}>0$ since $\gamma<x_{2}$.

2.3.1. $w=\left(0, w_{2}\right), w_{2}>0$.

$$
\begin{aligned}
F\left(d^{*}, w\right) & =u_{1}\left(d_{1}, w_{2}\right)+u_{2}\left(0, \frac{\varepsilon}{4}\right)-u_{1}\left(0, w_{2}\right)-u_{2}\left(0, w_{2}\right) \\
& =u_{1}\left(d_{1}, w_{2}\right)+\left(\mu v-\frac{\varepsilon}{4}\right)-0-\left(\mu v-w_{2}\right) \\
& =u_{1}\left(d_{1}, w_{2}\right)-\frac{\varepsilon}{4}+w_{2}
\end{aligned}
$$

implying that

$$
\begin{array}{rlr}
F\left(d^{*}, w\right)-G(d, x) & =\left(u_{1}\left(d_{1}, w_{2}\right)-\frac{\varepsilon}{4}+w_{2}\right)-\left(u_{1}\left(d_{1}, x_{2}\right)+(\lambda-\mu) v+x_{2}\right) \\
& =\left(u_{1}\left(d_{1}, w_{2}\right)-u_{1}\left(d_{1}, x_{2}\right)\right)-\frac{\varepsilon}{4}+(\mu-\lambda) v+\left(w_{2}-x_{2}\right) & \\
& >-\frac{\varepsilon}{4}-\frac{\varepsilon}{4}+(\mu-\lambda) v-\frac{\varepsilon}{4} & \text { (since } \left.\left|w_{2}-x_{2}\right|<\frac{\varepsilon}{4}\right) \\
& >-\varepsilon
\end{array}
$$

2.3.2. $w=\left(w_{1}, w_{2}\right), w_{1}>0, w_{2}>0$. Since

$F\left(d^{*}, w\right)=u_{1}\left(d_{1}, w_{2}\right)+u_{2}\left(w_{1}, \frac{\varepsilon}{4}\right)-u_{1}\left(w_{1}, w_{2}\right)-u_{2}\left(w_{1}, w_{2}\right)=u_{1}\left(d_{1}, w_{2}\right)+u_{2}\left(w_{1}, \frac{\varepsilon}{4}\right)-\left(v-w_{1}-w_{2}\right)$, 
we have

$$
\begin{aligned}
& F\left(d^{*}, w\right)-G(d, x) \\
& =\left[u_{1}\left(d_{1}, w_{2}\right)+u_{2}\left(w_{1}, \frac{\varepsilon}{4}\right)-\left(v-w_{1}-w_{2}\right)\right] \\
& =\left[u_{1}\left(d_{1}, w_{2}\right)-u_{1}\left(d_{1}, x_{2}\right)\right]+u_{2}\left(w_{1}, \frac{\varepsilon}{4}\right)-v+w_{1}+w_{2}-x_{2}+(\mu-\lambda) v \\
& >-\frac{\varepsilon}{4}+u_{2}\left(w_{1}, \frac{\varepsilon}{4}\right)-v+w_{1}+w_{2}-x_{2}+(\mu-\lambda) v \\
& =-\frac{\varepsilon}{4}+\pi_{2}\left(w_{1}, \frac{\varepsilon}{4}\right) v-\frac{\varepsilon}{4}-v+w_{1}+w_{2}-x_{2}+(\mu-\lambda) v \\
& >-\frac{\varepsilon}{4}+\pi_{2}\left(y_{1}\left(t, \frac{\varepsilon}{4}\right), \frac{\varepsilon}{4}\right) v-\frac{\varepsilon}{4}-v+w_{1}+w_{2}-x_{2}+(\mu-\lambda) v \\
& =-\frac{\varepsilon}{4}+v-\frac{\varepsilon}{4}-\frac{\varepsilon}{4}-v+w_{1}+w_{2}-x_{2}+(\mu-\lambda) v \\
& =-\frac{3 \varepsilon}{4}+w_{1}+\left(w_{2}-x_{2}\right)+(\mu-\lambda) v \\
& >-\frac{3 \varepsilon}{4}+w_{1}-\frac{\varepsilon}{4}+(\mu-\lambda) v \\
& >-\varepsilon
\end{aligned}
$$

2.4. $x=\left(x_{1}, x_{2}\right), x_{1}>0, x_{2}>0$ implies

$$
G(d, x)=u_{1}\left(d_{1}, x_{2}\right)+u_{2}\left(x_{1}, d_{2}\right)-u_{1}\left(x_{1}, 0\right)-u_{2}\left(x_{1}, x_{2}\right)=u_{1}\left(d_{1}, x_{2}\right)-\left(v-x_{1}-x_{2}\right) .
$$

Choose $0<\gamma<\min \left\{\frac{x_{1}}{2}, \frac{x_{2}}{2}\right\}$ so that $u_{1}\left(d_{1}, w_{2}\right)-u_{1}\left(d_{1}, x_{2}\right)>-\frac{\varepsilon}{4}$ for all $w_{2} \in\left(x_{2}-\gamma, x_{2}+\gamma\right) \cap\left[0, \alpha_{2}\right]$. Let $\delta:=\min \left\{\frac{\varepsilon}{4}, \gamma\right\}$ and choose $w \in N_{\delta}(x)$. Note that $w \in N_{\delta}(x)$ implies that each $w_{i}>0$ since $\gamma<x_{i}$. Therefore we need only consider the single case in which each $w_{i}>0$. In that case,

$$
F\left(d^{*}, w\right)=u_{1}\left(d_{1}, w_{2}\right)+u_{2}\left(w_{1}, \frac{\varepsilon}{4}\right)-\left(v-w_{1}-w_{2}\right)
$$

implying that

$$
\begin{aligned}
F\left(d^{*}, w\right)-G(d, x) & =u_{1}\left(d_{1}, w_{2}\right)+u_{2}\left(w_{1}, \frac{\varepsilon}{4}\right)-\left(v-w_{1}-w_{2}\right)-\left[u_{1}\left(d_{1}, x_{2}\right)-\left(v-x_{1}-x_{2}\right)\right] \\
& =\left[u_{1}\left(d_{1}, w_{2}\right)-u_{1}\left(d_{1}, x_{2}\right)\right]+u_{2}\left(w_{1}, \frac{\varepsilon}{4}\right)+\left(w_{1}-x_{1}\right)+\left(w_{2}-x_{2}\right) \\
& >-\frac{\varepsilon}{4}+u_{2}\left(w_{1}, \frac{\varepsilon}{4}\right)-\frac{\varepsilon}{4}-\frac{\varepsilon}{4} \\
& =\pi_{2}\left(w_{1}, \frac{\varepsilon}{4}\right) v-\varepsilon \\
& >-\varepsilon .
\end{aligned}
$$

Case 3. $0<d_{i} \leq \alpha_{i}$ for each $i$ so that $\left(\phi_{1}\left(d_{1}\right), \phi_{2}\left(d_{2}\right)\right)=d^{*}=d$.

3.1. $x=(0,0)$ implies

$G(d, x)=u_{1}\left(d_{1}, 0\right)+u_{2}\left(0, d_{2}\right)-u_{1}(0,0)-u_{2}(0,0)=\left(\mu v-d_{1}\right)+\left(\mu v-d_{2}\right)-\lambda v-\lambda v=2(\mu-\lambda) v-d_{1}-d_{2}$.

Let $\delta:=\min \left\{\frac{\varepsilon}{4}, y_{1}\left(t, d_{2}\right), y_{2}\left(t, d_{1}\right)\right\}$.

3.1.1. $w=(0,0)$.

$F\left(d^{*}, w\right)=u_{1}\left(d_{1}, 0\right)+u_{2}\left(0, d_{2}\right)-u_{1}(0,0)-u_{2}(0,0)=2(\mu-\lambda) v-\frac{\varepsilon}{2}=\mu v-d_{1}+\mu v-d_{2}-\lambda v-\lambda v$, 
implying that $F\left(d^{*}, w\right)-G(d, x)=0>-\varepsilon$.

3.1.2. $w=\left(w_{1}, 0\right), w_{1}>0$.

$$
\begin{aligned}
F\left(d^{*}, w\right) & =u_{1}\left(d_{1}, 0\right)+u_{2}\left(w_{1}, d_{2}\right)-u_{1}\left(w_{1}, 0\right)-u_{2}\left(w_{1}, 0\right) \\
& =\left(\mu v-d_{1}\right)+\left(\pi_{2}\left(w_{1}, d_{2}\right) v-d_{2}\right)-\left(\mu v-w_{1}\right)-\lambda v \\
& \left.>-d_{1}+\left(\pi_{2}\left(y_{1}\left(t, d_{2}\right), d_{2}\right) v-d_{2}\right)+w_{1}-\lambda v \quad \text { (since } w_{1}=\left|w_{1}-x_{1}\right|<y_{1}\left(t, d_{2}\right)\right) \\
& =(1-\lambda) v-d_{1}-\frac{\varepsilon}{4}-d_{2}+w_{1},
\end{aligned}
$$

implying that

$$
F\left(d^{*}, w\right)-G(d, x)=(1+\lambda-2 \mu) v-\frac{\varepsilon}{4}+w_{1}>-\varepsilon
$$

(since $1-2 \mu+\lambda \geq 0$ ).

3.1.3. $w=\left(w_{1}, w_{2}\right), w_{1}>0, w_{2}>0$.

$$
\begin{aligned}
F\left(d^{*}, w\right) & =u_{1}\left(d_{1}, w_{2}\right)+u_{2}\left(w_{1}, d_{2}\right)-u_{1}\left(w_{1}, w_{2}\right)-u_{2}\left(w_{1}, w_{2}\right) \\
& =u_{1}\left(d_{1}, w_{2}\right)+u_{2}\left(w_{1}, d_{2}\right)-\left(v-w_{1}-w_{2}\right) \\
& =\pi_{1}\left(d_{1}, w_{2}\right) v-d_{1}+\pi_{2}\left(w_{1}, d_{2}\right) v-d_{2}-v+w_{1}+w_{1} \\
& >\pi_{1}\left(d_{1}, y_{2}\left(t, d_{1}\right) v-d_{1}+\pi_{2}\left(y_{1}\left(t, d_{2}\right), d_{2}\right) v-d_{2}-v+w_{1}+w_{1}\right. \\
& =v-d_{1}-d_{2}+w_{1}+w_{1}-\frac{\varepsilon}{2}
\end{aligned}
$$

implying that

$F\left(d^{*}, w\right)-G(d, x)>\left(v-d_{1}-d_{2}+w_{1}+w_{1}-\frac{\varepsilon}{2}\right)-2(\mu-\lambda) v-d_{1}-d_{2}=(1-2(\mu-\lambda)) v+w_{1}+w_{1}-\frac{\varepsilon}{2}>-\varepsilon$ (since $1-2 \mu+\lambda \geq 0$ ).

3.2. $x=\left(x_{1}, 0\right), x_{1}>0$ implies

$$
G(d, x)=u_{1}\left(d_{1}, 0\right)+u_{2}\left(x_{1}, d_{2}\right)-u_{1}\left(x_{1}, 0\right)-u_{2}\left(x_{1}, 0\right)=u_{2}\left(x_{1}, d_{2}\right)-d_{1}+x_{1} \cdot{ }^{20}
$$

Choose $0<\gamma<\frac{x_{1}}{2}$ so that $u_{2}\left(w_{1}, d_{2}\right)-u_{2}\left(x_{1}, d_{2}\right)>-\frac{\varepsilon}{4}$ for all $w_{1} \in\left(x_{1}-\gamma, x_{1}+\gamma\right) \cap\left[0, \alpha_{1}\right]$. Let $\delta:=\min \left\{\frac{\varepsilon}{4}, \gamma, y_{2}\left(t, d_{1}\right)\right\}$ and choose $w \in N_{\delta}(x)$. Note that $w \in N_{\delta}(x)$ implies that $w_{1}>0$ since $\gamma<x_{2}$.

3.2.1. $w=\left(w_{1}, 0\right), w_{1}>0$.

$$
\begin{aligned}
F\left(d^{*}, w\right) & =u_{1}\left(d_{1}, 0\right)+u_{2}\left(w_{1}, d_{2}\right)-u_{1}\left(w_{1}, 0\right)-u_{2}\left(w_{1}, 0\right) \\
& =\left(\mu v-d_{1}\right)+u_{2}\left(w_{1}, d_{2}\right)-\left(\mu v-w_{1}\right)-0 \\
& =u_{2}\left(w_{1}, d_{2}\right)+w_{1}-d_{1}
\end{aligned}
$$

implying that

$$
\begin{aligned}
F\left(d^{*}, w\right)-G(d, x) & =\left(u_{2}\left(w_{1}, d_{2}\right)+w_{1}-d_{1}\right)-\left(u_{2}\left(x_{1}, d_{2}\right)-d_{1}+x_{1}\right) \\
& =\left(u_{2}\left(w_{1}, d_{2}\right)-u_{2}\left(x_{1}, d_{2}\right)\right)+\left(w_{1}-x_{1}\right) \\
& >-\frac{\varepsilon}{4}-\frac{\varepsilon}{4} \\
& >-\varepsilon
\end{aligned}
$$

3.2.2. $w=\left(w_{1}, w_{2}\right), w_{1}>0, w_{2}>0$.

$F\left(d^{*}, w\right)=u_{1}\left(d_{1}, w_{2}\right)+u_{2}\left(w_{1}, d_{2}\right)-u_{1}\left(w_{1}, w_{2}\right)-u_{2}\left(w_{1}, w_{2}\right)=u_{1}\left(d_{1}, w_{2}\right)+u_{2}\left(w_{1}, d_{2}\right)-\left(v-w_{1}-w_{2}\right)$,

\footnotetext{
${ }^{20}$ The case when $x=\left(0, x_{2}\right), x_{2}>0$ is symmetric to the case 3.2.
} 
implying that

$$
\begin{aligned}
& F\left(d^{*}, w\right)-G(d, x) \\
& =\left[\pi_{1}\left(d_{1}, w_{2}\right) v-d_{1}\right]+\left[u_{2}\left(w_{1}, d_{2}\right)-u_{2}\left(x_{1}, d_{2}\right)\right]-v+w_{1}+w_{2}+d_{1}-x_{1} \\
& >\pi_{1}\left(d_{1}, w_{2}\right) v-\frac{\varepsilon}{4}-v+w_{1}+w_{2}-x_{1} \\
& >\pi_{1}\left(d_{1}, y_{2}\left(t, d_{1}\right)\right) v-\frac{\varepsilon}{4}-v+w_{1}+w_{2}-x_{1} \\
& =v-\frac{\varepsilon}{4}-\frac{\varepsilon}{4}-v+w_{2}+\left(w_{1}-x_{1}\right) \\
& >-\frac{\varepsilon}{2}+w_{1}-\frac{\varepsilon}{4} \\
& >-\varepsilon
\end{aligned}
$$$$
\text { (since } \left.w_{2}=\left|w_{2}-x_{2}\right|<y_{2}\left(t, d_{1}\right)\right)
$$

3.3. $x=\left(x_{1}, x_{2}\right), x_{1}>0, x_{2}>0$ implies

$$
G(d, x)=u_{1}\left(d_{1}, x_{2}\right)+u_{2}\left(x_{1}, d_{2}\right)-u_{1}\left(x_{1}, x_{2}\right)-u_{2}\left(x_{1}, x_{2}\right)=u_{1}\left(d_{1}, x_{2}\right)+u_{2}\left(x_{1}, d_{2}\right)-\left(v-x_{1}-x_{2}\right) .
$$

Choose $0<\gamma<\min \left\{\frac{x_{1}}{2}, \frac{x_{2}}{2}\right\}$ so that $u_{1}\left(d_{1}, w_{2}\right)-u_{1}\left(d_{1}, x_{2}\right)>-\frac{\varepsilon}{4}$ for all $w_{2} \in\left(x_{2}-\gamma, x_{2}+\gamma\right) \cap\left[0, \alpha_{2}\right]$ and $u_{2}\left(w_{1}, d_{2}\right)-u_{2}\left(x_{1}, d_{2}\right)>-\frac{\varepsilon}{4}$ for all $w_{1} \in\left(x_{1}-\gamma, x_{1}+\gamma\right) \cap\left[0, \alpha_{1}\right]$. Let $\delta:=\min \left\{\frac{\varepsilon}{4}, \gamma\right\}$ and choose $w \in N_{\delta}(x)$. Note that $w \in N_{\delta}(x)$ implies that each $w_{i}>0$ since $\gamma<x_{i}$. Therefore we need only consider the single case in which each $w_{i}>0$. In that case,

$$
F\left(d^{*}, w\right)=u_{1}\left(d_{1}, w_{2}\right)+u_{2}\left(w_{1}, d_{2}\right)-\left(v-w_{1}-w_{2}\right),
$$

implying that

$$
\begin{aligned}
F\left(d^{*}, w\right)-G(d, x) & =u_{1}\left(d_{1}, w_{2}\right)+u_{2}\left(w_{1}, d_{2}\right)-\left(v-w_{1}-w_{2}\right)-\left[u_{1}\left(d_{1}, x_{2}\right)+u_{2}\left(x_{1}, d_{2}\right)-\left(v-x_{1}-x_{2}\right)\right] \\
& =\left[u_{1}\left(d_{1}, w_{2}\right)-u_{1}\left(d_{1}, x_{2}\right)\right]+\left[u_{2}\left(w_{1}, d_{2}\right)-u_{2}\left(x_{1}, d_{2}\right)\right]+\left(w_{1}-x_{1}\right)+\left(w_{2}-x_{2}\right) \\
& >-\frac{\varepsilon}{4}-\frac{\varepsilon}{4}-\frac{\varepsilon}{4}-\frac{\varepsilon}{4} \\
& >-\varepsilon
\end{aligned}
$$

This completes the proof.

\section{References}

[1] Aliprantis, C.D., and K.C. Border (2006), Infinite Dimensional Analysis. Berlin: SpringerVerlag.

[2] Allison, B.A., and J.J. Lepore (2014), "Verifying payoff security in the mixed extension of discontinuous games," Journal of Economic Theory 152, 291-303.

[3] Araujo, A., de Castro, L.I., and H. Moreira (2008), "Non-monotoniticies and the all-pay auction tie-breaking rule," Economic Theory 35, 407-440.

[4] Araujo, A., and L.I. de Castro (2009), "Pure strategy equilibria of single and double auctions with interdependent values," Games and Economic Behavior 65, 25-48.

[5] Athey, S. (2001), "Single crossing properties and the existence of pure strategy equilibria in games of incomplete information," Econometrica 69, 861-889.

[6] Balder, E.J. (1988), "Generalized equilibrium results for games with incomplete information," Mathematics of Operations Research 13, 265-276. 
[7] Balder, E.J. (1991), "On Cournot-Nash equilibrium distributions for games with differential information and discontinuous payoffs,” Economic Theory 1, 339-354.

[8] Balder, E.J., and A. Rustichini (1994), "An equilibrium result for games with private information and infinitely many players," Journal of Economic Theory 62, 385-393.

[9] Balder, E.J. (2000), "Lectures on Young measure theory and its applications in Economics," Rendiconti dell'Istituto di Matematica dell'Università di Trieste 31, Suppl. 1, $1-69$.

[10] Balder, E.J. (2001), “On ws-convergence of product measures," Mathematics of Operations Research 26, 494-518.

[11] Balder, E.J. (2002), "A unifying pair of Cournot-Nash equilibrium existence results," Journal of Economic Theory 102, 437-470.

[12] Balder, E.J. (2004), "An equilibrium existence result for games with incomplete information and indeterminate outcomes," Journal of Mathematical Economics 40, 297-320.

[13] Baye, M.R., G. Tian, and J. Zhou (1993), "Characterizations of the existence of equilibria in games with discontinuous and non-quasiconcave payoffs," Review of Economic Studies $60,935-948$.

[14] Baye, M.R., and J. Morgan (2002), "Winner-take-all price competition," Economic Theory $19,271-282$.

[15] Blavatskyy, P.R. (2010), "Contest success function with the possibility of a draw: Axiomatization," Journal of Mathematical Economics 46, 267-276.

[16] Brems, H. (1952), “A discontinuous cost function,” American Economic Review 42, 577586.

[17] Carbonell-Nicolau, O., and E.A. Ok (2007), "Voting of income taxation," Journal of Economic Theory 134, 249-286.

[18] Carbonell-Nicolau, O. (2014), "Semicontinuous integrands as jointly measurable maps," Commentationes Mathematicae Universitatis Carolinae 55, 189-193.

[19] Carmona, G. (2013), Existence and Stability of Nash Equilibrium. Singapore: World Scientific Publishing.

[20] Castaing, C., P.R. de Fitte, and M. Valadier (2004), Young Measures on Topological Spaces. Dordrecht/Boston/London: Kluwer Academic Publishers.

[21] de Castro, L.I. (2009), "Affiliation and dependence in economic models," mimeo.

[22] Clarke, R. (1983), "Collusion and the incentives for information sharing," Bell Journal of Economics 14, 383-394.

[23] Dasgupta, P., and E. Maskin (1986), "The existence of equilibrium in discontinuous economic games, I: Theory," Review of Economic Studies 53, 1-26.

[24] Dasgupta, P., and E. Maskin (1986), "The existence of equilibrium in discontinuous economic games, II: Applications," Review of Economic Studies 53, 27-41. 
[25] Dudley, R.M. (2003), Real Analysis and Probability. Cambridge, MA: Cambridge University Press.

[26] Daughety, A.F. (2008), “Cournot competition.” In: S.N. Durlauf and L.E. Blume (Eds.), The New Palgrave Dictionary of Economics, Second Edition. Palgrave Macmillan.

[27] Edgeworth, F. (1925). Papers Relating to Political Economy. London: MacMillan.

[28] Einy, E., Moreno, D., and B. Shitovitz (2002), "Information advantage in Cournot oligopoly," Journal of Economic Theory 106, 151-160.

[29] Einy, E., Moreno, D., and B. Shitovitz (2003), "The value of public information in a Cournot duopoly," Games and Economic Behavior 44, 272-285.

[30] Einy, E., Haimanko, O., Moreno, D., and B. Shitovitz (2010), "On the existence of Bayesian Cournot equilibrium," Games and Economic Behavior 68, 77-94.

[31] Friedman, J.W. (1972), "Duality principles in the theory of cost and production revisited," International Economic Review 13, 167-170.

[32] Gal-Or, E. (1985), “Information sharing in oligopoly," Econometrica 53, 329-343.

[33] Gal-Or, E. (1986), "Information transmission-Cournot and Bertrand equilibria," Review of Economic Studies 53, 85-92.

[34] He, W., and N.C. Yannelis (2015), "Discontinuous games with asymmetric information: An extension of Reny's existence theorem," Games and Economic Behavior 91, 26-35.

[35] He, W., and N.C. Yannelis (2015), "On the existence of equilibria in discontinuous Bayesian games," mimeograph.

[36] Horvath, J. (1966), Topological Vector Spaces and Distributions. Reading, MA: AddisonWesley.

[37] Jackson, M.O. (2009), "Non-existence of equilibrium in Vickrey, second-price, and English auctions," Review of Economic Design 13, 137-145.

[38] Jackson, M.O., L.K. Simon, J.M. Swinkels, and W.R. Zame (2002), "Communication and equilibrium in discontinuous games of incomplete information," Econometrica 70, 1711-1740.

[39] Jackson, M.O., and J.M. Swinkels (2005), "Existence of equilibrium in single and double private value auctions," Econometrica 73, 93-139.

[40] Krishna, V., and J. Morgan (1997), "An analysis of the war of attrition and the all-pay auction," Journal of Economic Theory 72, 343-362.

[41] Lebrun, B. (1999), "First price auctions in the asymmetric $N$ bidder case," International Economic Review 40, 125-142.

[42] Lepore, J.J. (2008), "Cournot and Bertrand-Edgeworth competition when rivals' costs are unknown,” Economics Letters 101, 237-240.

[43] Lepore, J.J. (2012), "Cournot outcomes under Bertrand-Edgeworth competition with demand uncertainty, Journal of Mathematical Economics 48, 177-186. 
[44] Levitan, R., and M. Shubik (1972), "Price Duopoly and Capacity Constraints," International Economic Review 13, 111-122.

[45] Lizzeri, A., and N. Persico (2000), "Uniqueness and existence of equilibrium in auctions with a reserve price," Games and Economic Behavior 30, 83-114.

[46] Maskin, E. (1986), "The existence of equilibrium with price-setting firms," American Economic Review, Papers and Proceedings 76, 382-386.

[47] Maskin, E., and J. Riley (2000), "Equilibrium in sealed high bid auctions," Review of Economic Studies 67, 439-454.

[48] McAdams, D. (2003), "Isotone equilibrium in games of incomplete information," Econometrica 71, 1191-1214.

[49] Milgrom, P.R., and R.J. Weber (1982), "A theory of auctions and competitive bidding," Econometrica 50, 1089-1122.

[50] Milgrom, P.R., and R.J. Weber (1985), "Distributional strategies for games with incomplete information," Mathematics of Operations Research 10, 619-632.

[51] Monteiro, P.K., and F.H. Page (2007), "Uniform payoff security and Nash equilibrium in compact games," Journal of Economic Theory 134, 566-575.

[52] Monteiro, P.K., and H. Moreira (2006), "First-price auctions without affiliation," Economics Letters 91, 1-7.

[53] Nessah, R., and G. Tian (2013), "Existence of solution of minimax inequalities, equilibria in games and fixed points without convexity and compactness assumptions," Journal of Optimization Theory and Applications 157, 75-95.

[54] Nitzan, Sh. (1991), “Collective rent dissipation,” Economic Journal 101, 1522-1534.

[55] Nitzan, Sh. (1994), "Modelling rent-Seeking contests," European Journal of Political Economy 10, 41-60.

[56] Novshek, W., and H. Sonnenschein (1982), "Fulfilled expectations Cournot duopoly with information acquisition and release," Bell Journal of Economics 13, 214-218.

[57] Nti, K. (1997), “Comparative statics of contests and rent-seeking games," International Economic Review 38, 43-59.

[58] Prokopovych, P., and N.C. Yannelis (2014), "On the existence of mixed strategy Nash equilibria,” Journal of Mathematical Economics 52, 87-97.

[59] Raith, M. (1996), "A general model of information sharing in oligopoly," Journal of Economic Theory 71, 260-288.

[60] Reny, P.J. (1999), "On the existence of pure and mixed strategy Nash equilibria in discontinuous games," Econometrica 67, 1029-1056.

[61] Reny, P.J. (2011), "On the existence of monotone pure-strategy equilibria in bayesian games," Econometrica 79, 499-553.

[62] Reny, P.J., and S. Zamir (2004), "On the existence of pure strategy monotone equilibria in asymmetric first-price auctions," Econometrica 72, 1105-1125. 
[63] Reynolds, S., and B. Wilson (2000), "Bertrand-Edgeworth competition, demand uncertainty, and asymmetric outcomes," Journal of Economic Theory 92, 122-141.

[64] Rieder, U. (1978), "Measurable selection theorems for optimization problems," Manuscripta Mathematica 24, 115-131.

[65] Rosen, S. (1986), "Prizes and incentives in elimination tournaments," American Economic Review 76, 701-715.

[66] Sakai, Y. (1985), "The value of information in a simple duopoly model," Journal of Economic Theory 36, 36-54.

[67] Sakai, Y. (1986), "Cournot and Bertrand equilibria under imperfect information," Journal of Economics (Zeitschrift für Nationalökonomie) 46, 213-232.

[68] Schäl, M. (1975), "On dynamic programming: compactness of the space of policies," Stochastic Processes and their Applications 3, 345-364.

[69] Shapiro, C. (1986), "Exchange of cost information in oligopoly," Review of Economic Studies 53, 433-446.

[70] Shubik, M. (1955), "A comparison of treatments of a duopoly problem (Part II)," Econometrica 23, 417-431.

[71] Simon, L.K. (1987), "Games with discontinuous payoffs," Review of Economic Studies $54,569-597$.

[72] Staiger, R., and F. Wolak (1992), "Collusive pricing with capacity constraints in the presence of demand uncertainty," Rand Journal of Economics 23, 203-220.

[73] Szymanski S. (2003), "The economic design of sporting contests," Journal of Economic Literature 41, 1137-1187.

[74] Tullock, G. (1980), "Efficient rent-seeking." In: Buchanan, J.M., R.D. Tollison, and G. Tullock (Eds.), Toward a Theory of the Rent-Seeking Society. College Station: Texas A\&M Press.

[75] Vives, X. (1984), "Duopoly information equilibrium: Cournot and Bertrand," Journal of Economic Theory 34, 71-94.

[76] Vives, X. (1988), “Aggregation of information in large Cournot markets," Econometrica $56,851-876$.

[77] Vives, X. (1999). Oligopoly Pricing. Old Ideas and New Tools. Cambridge, MA: The MIT Press.

[78] Wasser, C. (2013), "A note on Bayesian Nash equilibria in imperfectly discriminating contests," Mathematical Social Sciences 66, 180-182.

[79] Yannelis, N.C., and A. Rustichini (1991), "Equilibrium points of non-cooperative random and Bayesian games.” In: Aliprantis, C.D., K.C. Border, and W.A.J. Luxemburg (Eds.), Positive Operators, Riesz Spaces, and Economics. Berlin-Heidelberg: Springer-Verlag. 